Network Working Group

Request for Comments: 2446

Category: Standards Track
S. Silverberg

Microsoft

S. Mansour

Netscape

F. Dawson

Lotus

R. Hopson

ON Technologies

November 1998

\title{
iCalendar Transport-Independent Interoperability Protocol \\ (iTIP) \\ Scheduling Events, BusyTime, To-dos and Journal Entries
}

Status of this Memo

This document specifies an Internet standards track protocol for the Internet community, and requests discussion and suggestions for improvements. Please refer to the current edition of the "Internet Official Protocol Standards" (STD 1) for the standardization state and status of this protocol. Distribution of this memo is unlimited.

Copyright Notice

Copyright (C) The Internet Society (1998). All Rights Reserved.

Abstract

This document specifies how calendaring systems use icalendar objects to interoperate with other calendar systems. It does so in a general way so as to allow multiple methods of communication between systems. Subsequent documents specify interoperable methods of communications between systems that use this protocol.

The document outlines a model for calendar exchange that defines both static and dynamic event, to-do, journal and free/busy objects. Static objects are used to transmit information from one entity to another without the expectation of continuity or referential integrity with the original item. Dynamic objects are a superset of static objects and will gracefully degrade to their static counterparts for clients that only support static objects.

This document specifies an Internet protocol based on the icalendar object specification that provides scheduling interoperability between different calendar systems. The Internet protocol is called the "iCalendar Transport-Independent Interoperability Protocol (iTIP)". 
iTIP complements the iCalendar object specification by adding semantics for group scheduling methods commonly available in current calendar systems. These scheduling methods permit two or more calendar systems to perform transactions such as publish, schedule, reschedule, respond to scheduling requests, negotiation of changes or cancel icalendar-based calendar components.

iTIP is defined independent of the particular transport used to transmit the scheduling information. Companion memos to iTIP provide bindings of the interoperability protocol to a number of Internet protocols.

Table of Contents

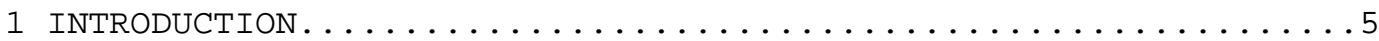

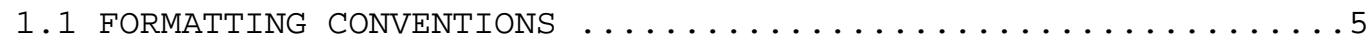

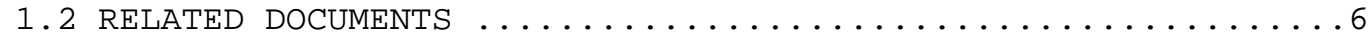

1.3 ITIP ROLES AND TRANSACTIONS $\ldots \ldots \ldots \ldots \ldots \ldots \ldots \ldots \ldots \ldots \ldots \ldots \ldots$

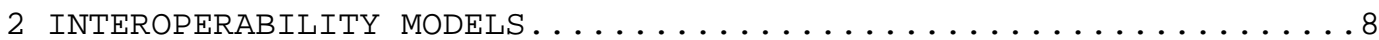

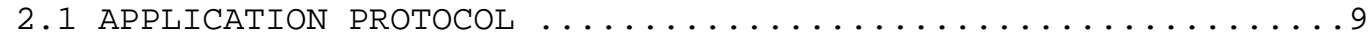

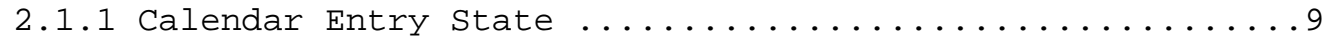

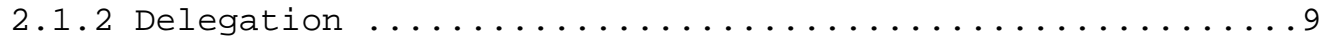

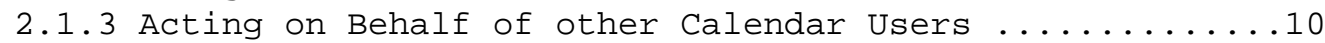

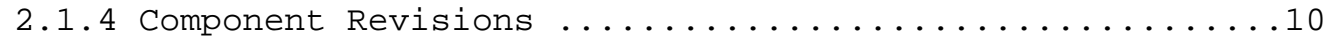

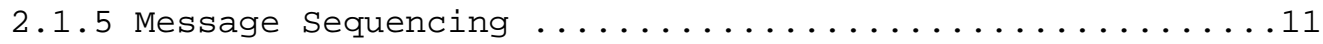

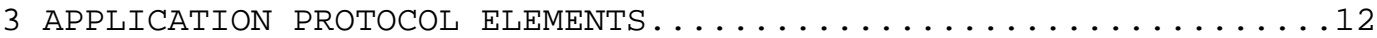

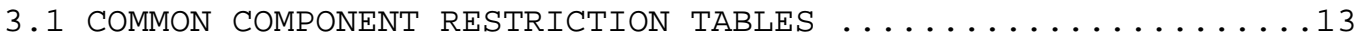

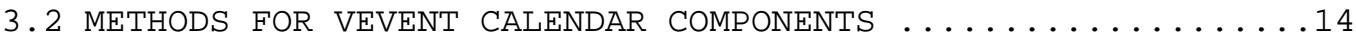

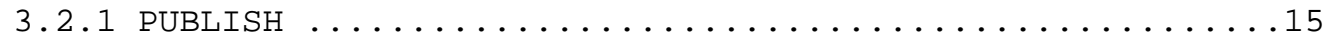

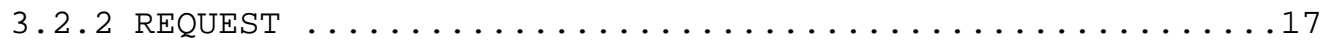

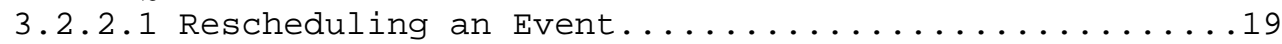

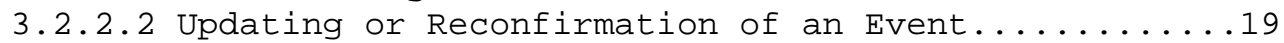

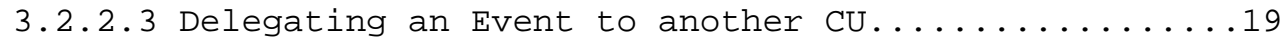

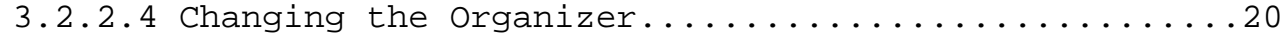

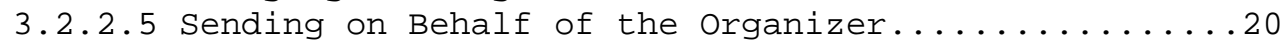

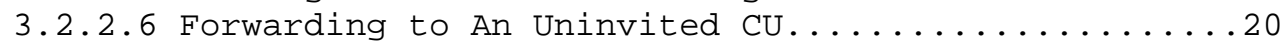

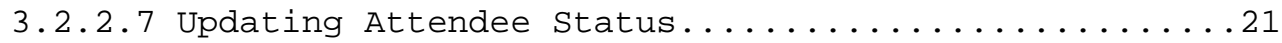

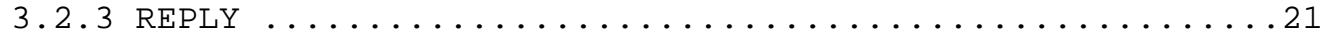

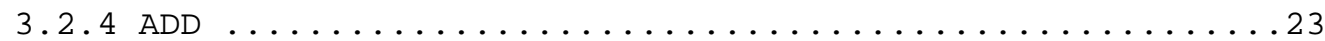

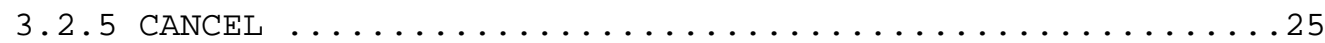

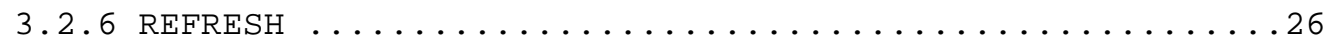

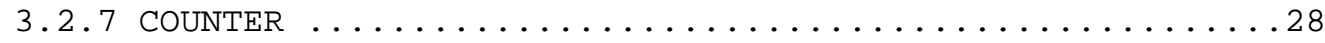

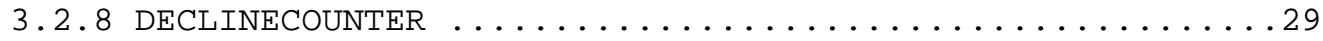

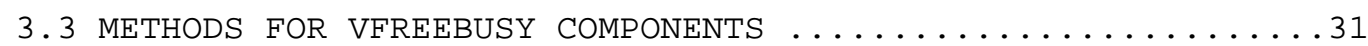

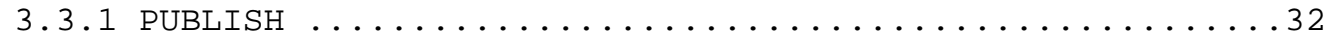

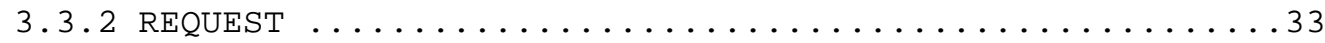

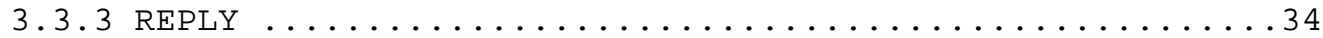

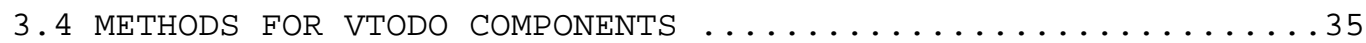




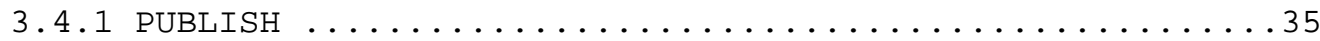

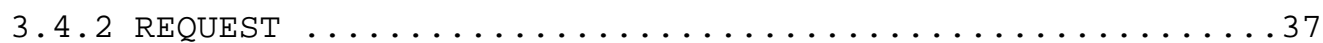

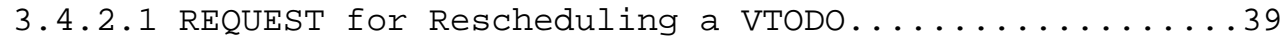

3.4.2.2 REQUEST for Update or Reconfirmation of a VTODO....39

3.4.2.3 REQUEST for Delegating a VTODO..............40

3.4.2.4 REQUEST Forwarded To An Uninvited Calendar User...40

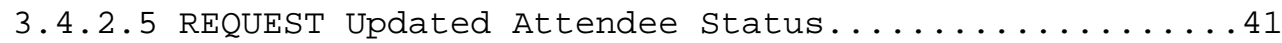

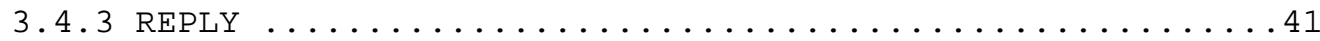

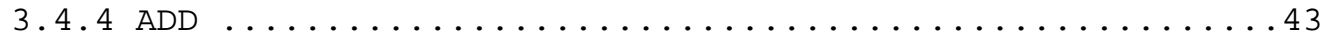

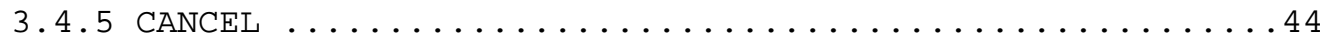

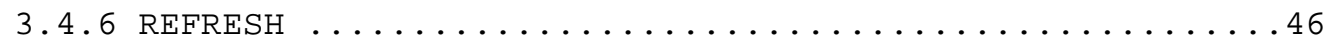

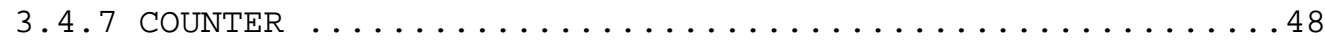

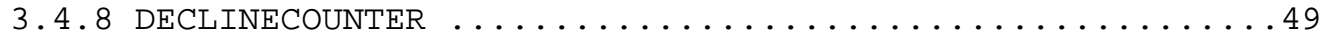

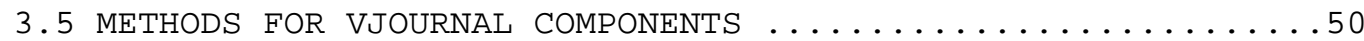

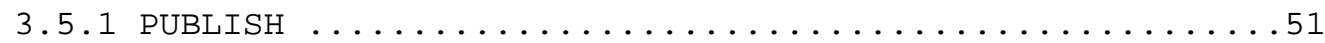

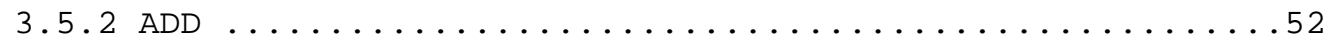

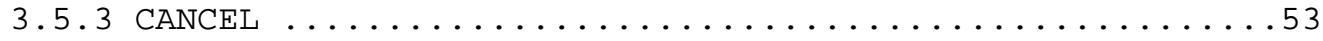

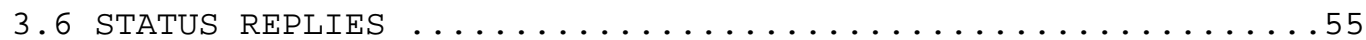

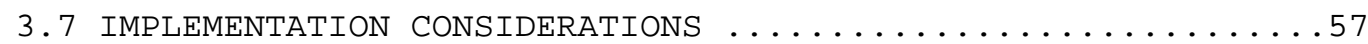

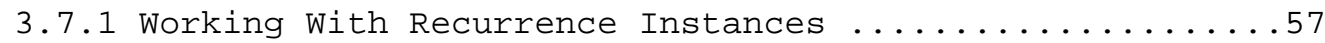

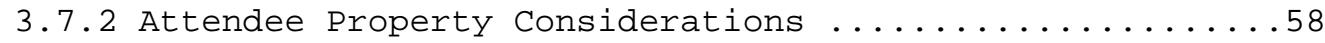

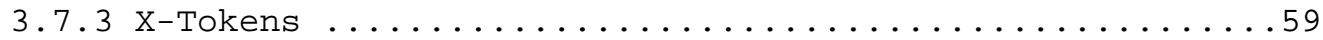

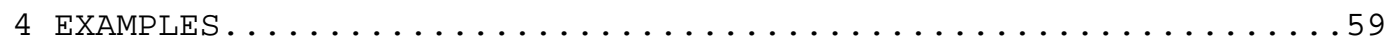

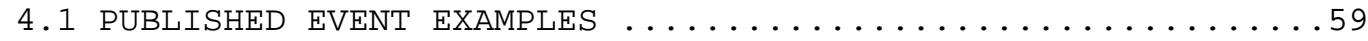

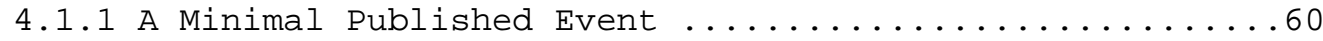

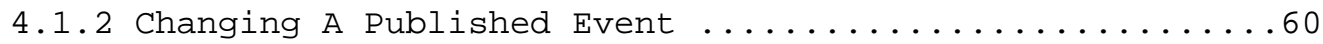

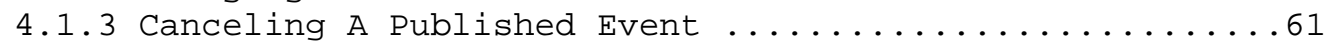

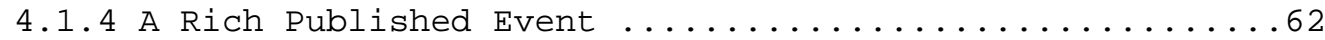

4.1.5 Anniversaries or Events attached to entire days .....63

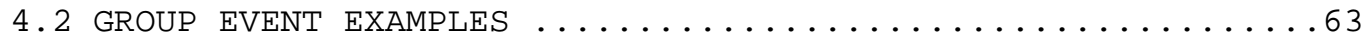

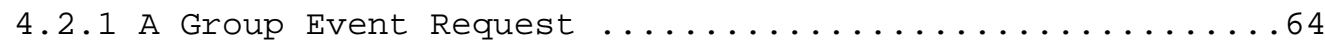

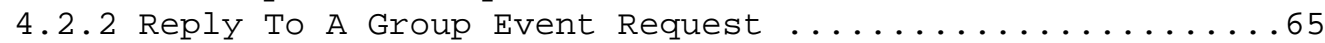

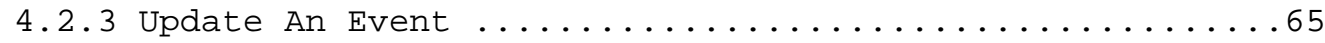

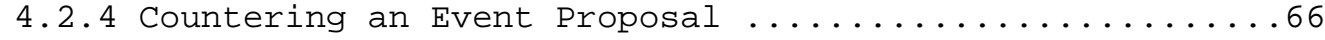

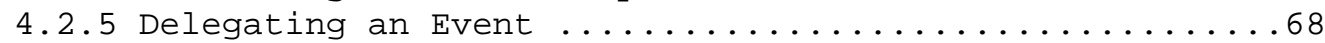

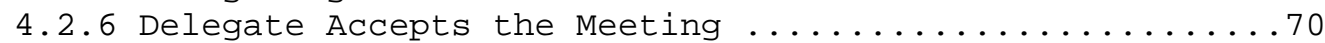

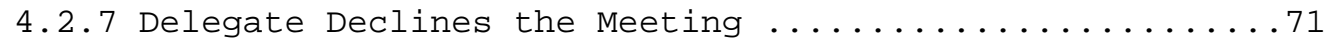

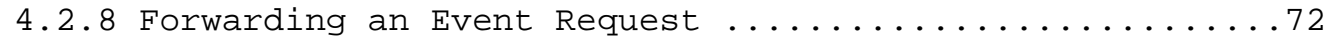

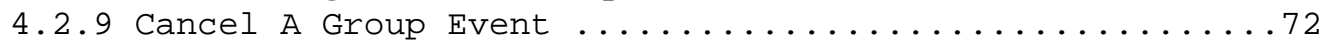

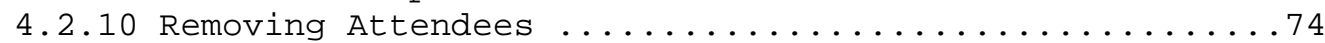

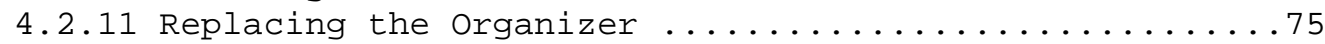

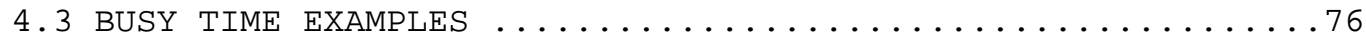

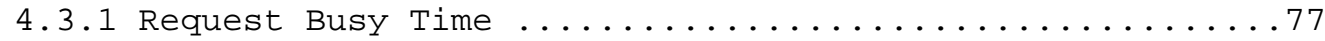

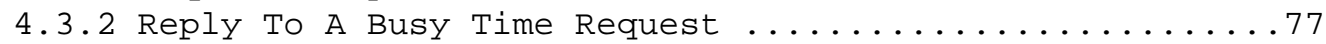

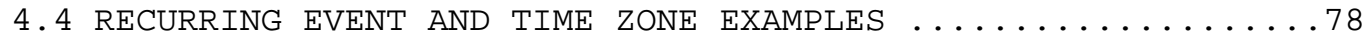

4.4.1 A Recurring Event Spanning Time Zones .............78

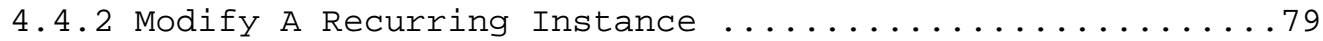

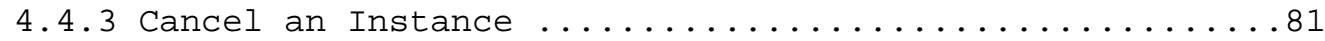




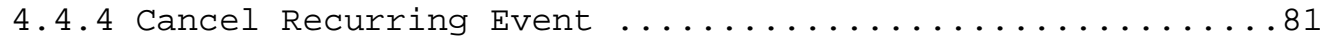

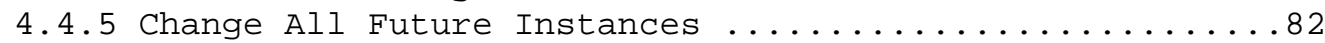

4.4 .6 Add A New Instance To A Recurring Event ...........82

4.4.7 Add A New Series of Instances To A Recurring Event ....83

4.4.8 Counter An Instance of A Recurring Event .........87

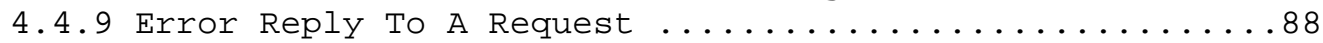

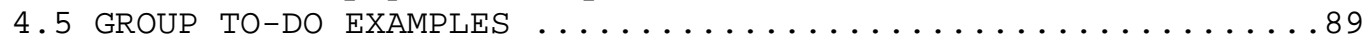

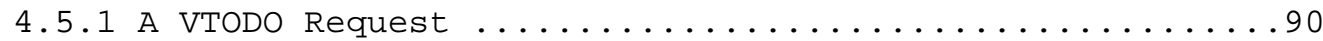

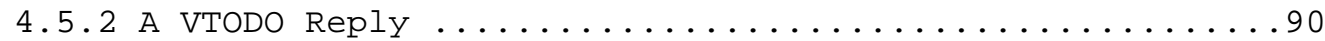

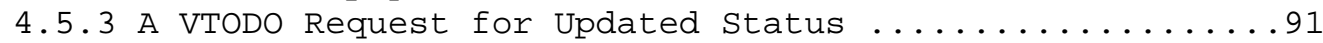

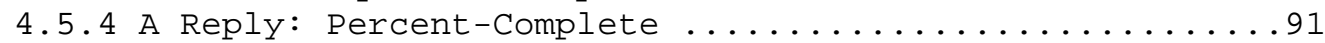

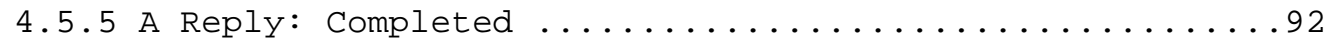

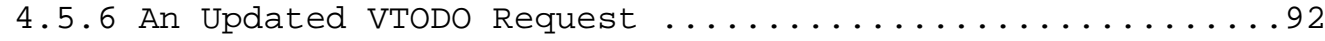

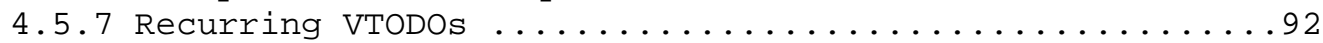

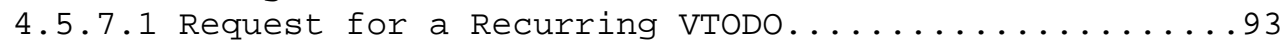

4.5.7.2 Calculating due dates in recurring VTODOs.......93

4.5.7.3 Replying to an instance of a recurring VTODO.....93

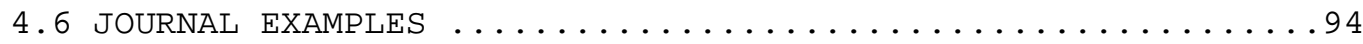

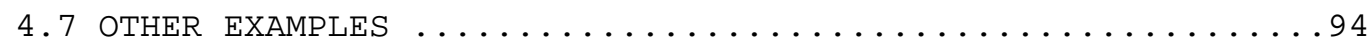

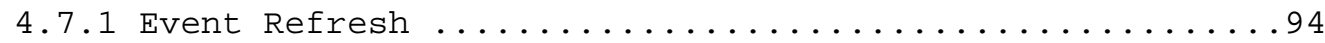

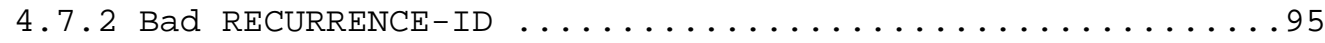

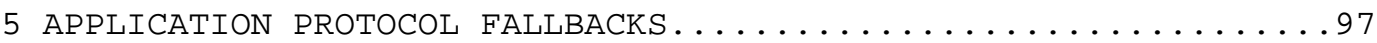

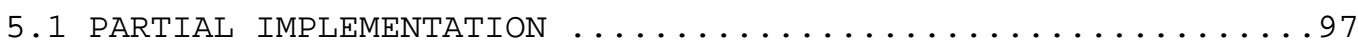

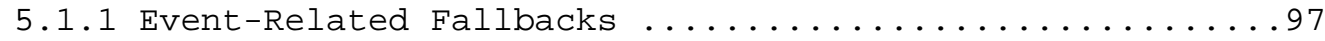

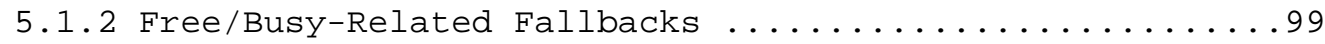

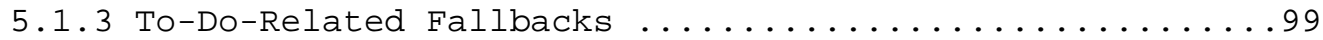

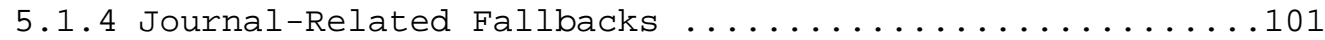

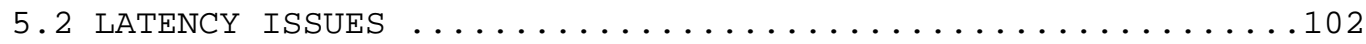

5.2.1 Cancellation of an Unknown Calendar Component. .....102

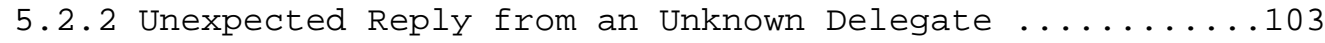

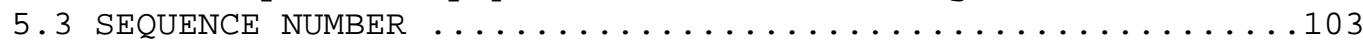

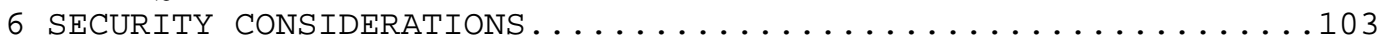

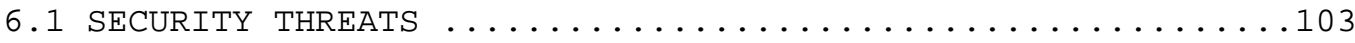

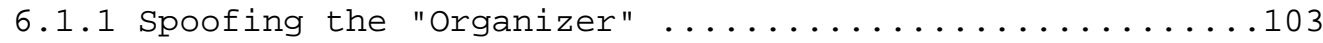

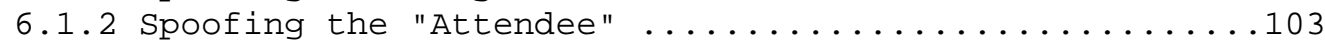

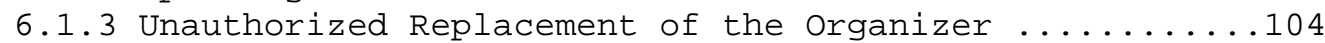

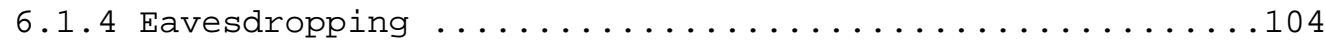

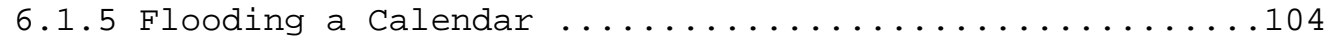

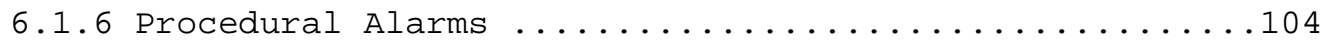

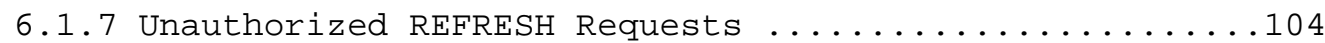

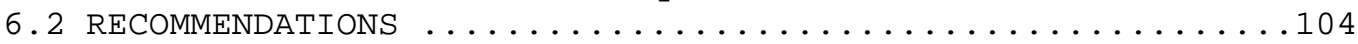

6.2.1 Use of [RFC-1847] to secure iTIP transactions ......105

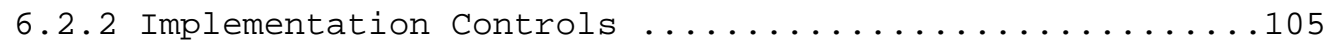

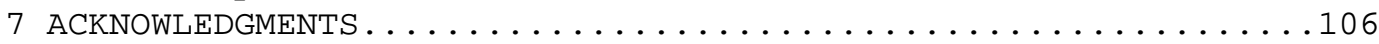

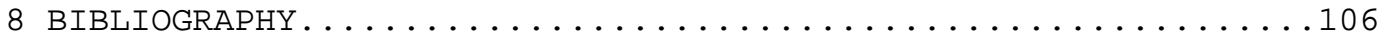

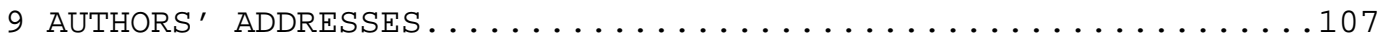

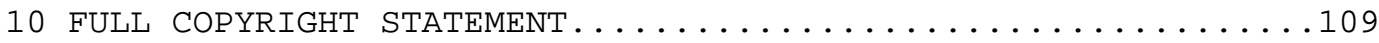

Silverberg, et. al. Standards Track [Page 4] 


\section{Introduction}

This document specifies how calendaring systems use icalendar objects to interoperate with other calendar systems. In particular, it specifies how to schedule events, to-dos, or daily journal entries. It further specifies how to search for available busy time information. It does so in a general way so as to allow multiple methods of communication between systems. Subsequent documents specify transport bindings between systems that use this protocol.

This protocol is based on messages sent from an originator to one or more recipients. For certain types of messages, a recipient may reply, in order to update their status and may also return transaction/request status information. The protocol supports the ability for the message originator to modify or cancel the original message. The protocol also supports the ability for recipients to suggest changes to the originator of a message. The elements of the protocol also define the user roles for its transactions.

\subsection{Formatting Conventions}

In order to refer to elements of the calendaring and scheduling model, core object or interoperability protocol defined in [iCAL] and [iTIP] several formatting conventions have been utilized.

The key words "MUST", "MUST NOT", "REQUIRED", "SHALL", "SHALL NOT", "SHOULD", "SHOULD NOT", "RECOMMENDED", "MAY" and "OPTIONAL" in this document are to be interpreted as described in [RFC-2119].

Calendaring and scheduling roles are referred to in quoted-strings of text with the first character of each word in upper case. For example, "Organizer" refers to a role of a "Calendar User" (CU) within the scheduling protocol defined by [iTIP]. Calendar components defined by [iCAL] are referred to with capitalized, quoted-strings of text. All calendar components start with the letter "V". For example, "VEVENT" refers to the event calendar component, "VTODO" refers to the to-do calendar component and "VJOURNAL" refers to the daily journal calendar component. Scheduling methods defined by [iTIP] are referred to with capitalized, quoted-strings of text. For example, "REQUEST" refers to the method for requesting a scheduling calendar component be created or modified, "REPLY" refers to the method a recipient of a request uses to update their status with the "Organizer" of the calendar component.

Properties defined by [iCAL] are referred to with capitalized, quoted-strings of text, followed by the word "property". For example, "ATTENDEE" property refers to the icalendar property used to convey the calendar address of a "Calendar User". Property parameters 
defined by this memo are referred to with lower case, quoted-strings of text, followed by the word "parameter". For example, "value" parameter refers to the icalendar property parameter used to override the default data type for a property value. Enumerated values defined by this memo are referred to with capitalized text, either alone or followed by the word "value".

In tables, the quoted-string text is specified without quotes in order to minimize the table length.

\subsection{Related Documents}

Implementers will need to be familiar with several other memos that, along with this one, describe the Internet calendaring and scheduling standards. This document, [iTIP], specifies an interoperability protocol for scheduling between different implementations. The related documents are:

[iCAL] - specifies the objects, data types, properties and property parameters used in the protocols, along with the methods for representing and encoding them;

[iMIP] specifies an Internet email binding for [iTIP].

This memo does not attempt to repeat the specification of concepts or definitions from these other memos. Where possible, references are made to the memo that provides for the specification of these concepts or definitions.

\subsection{ITIP Roles and Transactions}

ITIP defines methods for exchanging [iCAL] objects for the purposes of group calendaring and scheduling between "Calendar Users" (CUs) . CUs take on one of two roles in iTIP. The CU who initiates an exchange takes on the role of "Organizer". For example, the CU who proposes a group meeting is the "Organizer". The CUs asked to participate in the group meeting by the "Organizer" take on the role of "Attendee". Note that "role" is also a descriptive parameter to the _ATTENDEE_ property. Its use is to convey descriptive context to an "Attendee" such as "chair", "req-participant" or "non-participant" and has nothing to do with the calendaring workflow. 
The ITIP methods are listed below and their usage and semantics are defined in section 3 of this document.

\begin{tabular}{|c|c|}
\hline Method & Description \\
\hline \multicolumn{2}{|c|}{ 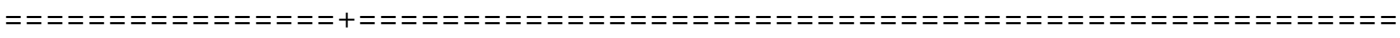 } \\
\hline PUBLISH & $\begin{array}{l}\text { Used to publish a calendar entry to one or more } \\
\text { Calendar Users. There is no interactivity } \\
\text { between the publisher and any other calendar } \\
\text { user. An example might include a baseball team } \\
\text { publishing its schedule to the public. }\end{array}$ \\
\hline REQUEST & $\begin{array}{l}\text { Used to schedule a calendar entry with other } \\
\text { Calendar Users. Requests are interactive in that } \\
\text { they require the receiver to respond using } \\
\text { the Reply methods. Meeting Requests, Busy } \\
\text { Time requests and the assignment of VToDos to } \\
\text { other Calendar Users are all examples. } \\
\text { Requests are also used by the "Organizer" to } \\
\text { update the status of a calendar entry. }\end{array}$ \\
\hline REPLY & $\begin{array}{l}\text { A Reply is used in response to a Request to } \\
\text { convey "Attendee" status to the "Organizer". } \\
\text { Replies are commonly used to respond to meeting } \\
\text { and task requests. }\end{array}$ \\
\hline $\mathrm{ADD}$ & $\begin{array}{l}\text { Add one or more instances to an existing } \\
\text { VEVENT, VTODO, or VJOURNAL. }\end{array}$ \\
\hline CANCEL & $\begin{array}{l}\text { Cancel one or more instances of an existing } \\
\text { VEVENT, VTODO, or VJOURNAL. }\end{array}$ \\
\hline REFRESH & $\begin{array}{l}\text { The Refresh method is used by an "Attendee" to } \\
\text { request the latest version of a calendar entry. }\end{array}$ \\
\hline COUNTER & $\begin{array}{l}\text { The Counter method is used by an "Attendee" to } \\
\text { negotiate a change in the calendar entry. } \\
\text { Examples include the request to change a } \\
\text { proposed Event time or change the due date for a } \\
\text { VToDo. }\end{array}$ \\
\hline $\begin{array}{l}\text { DECLINE- } \\
\text { COUNTER }\end{array}$ & $\begin{array}{l}\text { Used by the "Organizer" to decline the proposed } \\
\text { counter-proprosal. }\end{array}$ \\
\hline
\end{tabular}


Group scheduling in iTIP is accomplished using the set of "request" and "response" methods described above. The following table shows the methods broken down by who can send them.

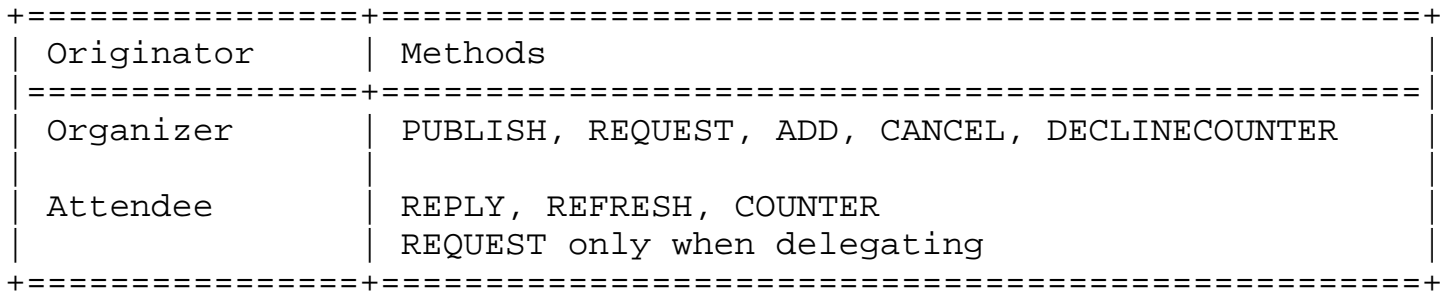

Note that for some calendar component types, the allowable methods are a subset of the above set.

2 Interoperability Models

There are two distinct protocols relevant to interoperability: an "Application Protocol" and a "Transport Protocol". The Application Protocol defines the content of the iCalendar objects sent between sender and receiver to accomplish the scheduling transactions listed above. The Transport Protocol defines how the iCalendar objects are sent between the sender and receiver. This document focuses on the Application Protocol. Binding documents such as [iMIP] focus on the Transport Protocol.

The connection between Sender and Receiver in the diagram below refers to the Application Protocol. The iCalendar objects passed from the Sender to the Receiver are presented in Section 3, Application Protocol Elements.

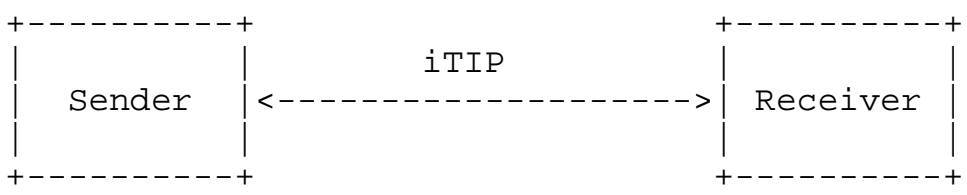

There are several variations of this diagram in which the Sender and Receiver take on various roles of a "Calendar User Agent" (CUA) or a "Calendar Service" (CS).

The architecture of iTIP is depicted in the diagram below. An application written to this specification may work with bindings for the store-and-forward transport, the real time transport, or both. Also note that iTIP could be bound to other transports. 


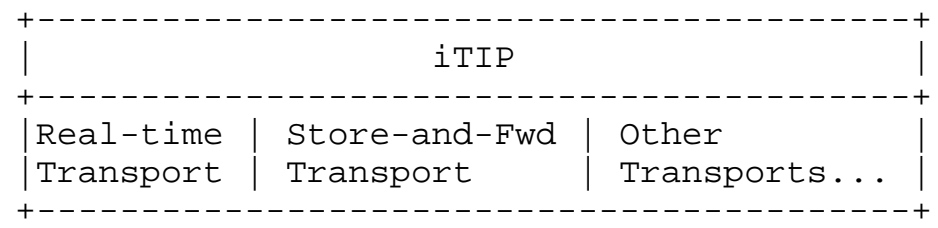

\subsection{Application Protocol}

In the iTIP model, a calendar entry is created and managed by an "Organizer". The "Organizer" interacts with other CUs by sending one or more of the iTIP messages listed above. "Attendees" use the "REPLY" method to communicate their status. "Attendees" do not make direct changes to the master calendar entry. They can, however, use the "COUNTER" method to suggest changes to the "Organizer". In any case, the "Organizer" has complete control over the master calendar entry.

\subsubsection{Calendar Entry State}

There are two distinct states relevant to calendar entries: the overall state of the entry and the state associated with an

"Attendee" to that entry.

The state of an entry is defined by the "STATUS" property and is controlled by the "Organizer." There is no default value for the "STATUS" property. The "Organizer" sets the "STATUS" property to the appropriate value for each calendar entry.

The state of a particular "Attendee" relative to an entry is defined by the "partstat" parameter in the "ATTENDEE" property for each "Attendee". When an "Organizer" issues the initial entry, "Attendee" status is unknown. The "Organizer" specifies this by setting the "partstat" parameter to "NEEDS-ACTION". Each "Attendee" modifies their "ATTENDEE" property "partstat" parameter to an appropriate value as part of a "REPLY" message sent back to the "Organizer".

\subsubsection{Delegation}

Delegation is defined as the process by which an "Attendee" grants another CU (or several CUs) the right to attend on their behalf. The "Organizer" is made aware of this change because the delegating "Attendee" informs the "Organizer". These steps are detailed in the REQUEST method section. 


\subsubsection{Acting on Behalf of other Calendar Users}

In many organizations one user will act on behalf of another to organize and/or respond to meeting requests. ITIP provides two mechanisms that support these activities.

First, the "Organizer" is treated as a special entity, separate from "Attendees". All responses from "Attendees" flow to the "Organizer", making it easy to separate a calendar user organizing a meeting from calendar users attending the meeting. Additionally, icalendar provides descriptive roles for each "Attendee". For instance, a role of "chair" may be ascribed to one or more "Attendees". The "chair" and the "Organizer" may or may not be the same calendar user. This maps well to scenarios where an assistant may manage meeting logistics for another individual who chairs a meeting.

Second, a "sent-by" parameter may be specified in either the "Organizer" or "Attendee" properties. When specified, the "sent-by" parameter indicates that the responding $\mathrm{CU}$ acted on behalf of the specified "Attendee" or "Organizer".

\subsubsection{Component Revisions}

The "SEQUENCE" property is used by the "Organizer" to indicate revisions to the calendar component. The rules for incrementing the "SEQUENCE" number are defined in [iCAL]. For clarity, these rules are paraphrased here in terms of how they are applied in [iTIP]. For a given "UID" in a calendar component:

- For the "PUBLISH" and "REQUEST" methods, the "SEQUENCE" property value is incremented according to the rules defined in [iCAL].

- The "SEQUENCE" property value MUST be incremented each time the "Organizer" uses the "ADD" or "CANCEL" methods.

- The "SEQUENCE" property value MUST NOT be incremented when using "REPLY", "REFRESH", "COUNTER", "DECLINECOUNTER", or when sending a delegation "REQUEST".

In some circumstances the "Organizer" may not have received responses to the final revision sent out. In this situation, the "Organizer" may wish to send an update "REQUEST", and set "RSVP=TRUE" for all "Attendees", so that current responses can be collected. 
The value of the "SEQUENCE" property contained in a response from an "Attendee" may not always match the "Organizer's" revision. Implementations may choose to have the CUA indicate to the CU that the response is to an entry that has been revised and allow the CU to decide whether or not to accept the response.

\subsubsection{Message Sequencing}

CUAs that handle the [iTIP] application protocol must often correlate a component in a calendar store with a component received in the [iTIP] message. For example, an event may be updated with a later revision of the same event. To accomplish this, a CUA must correlate the version of the event already in its calendar store with the version sent in the [iTIP] message. In addition to this correlation, there are several factors that can cause [iTIP] messages to arrive in an unexpected order. That is, an "Organizer" could receive a reply to an earlier revision of a component AFTER receiving a reply to a later revision.

To maximize interoperability and to handle messages that arrive in an unexpected order, use the following rules:

1. The primary key for referencing a particular iCalendar component is the "UID" property value. To reference an instance of a recurring component, the primary key is composed of the "UID" and the "RECURRENCE-ID" properties.

2. The secondary key for referencing a component is the "SEQUENCE" property value. For components where the "UID" is the same, the component with the highest numeric value for the "SEQUENCE" property obsoletes all other revisions of the component with lower values.

3. "Attendees" send "REPLY" messages to the "Organizer". For replies where the "UID" property value is the same, the value of the "SEQUENCE" property indicates the revision of the component to which the "Attendee" is replying. The reply with the highest numeric value for the "SEQUENCE" property obsoletes all other replies with lower values.

4. In situations where the "UID" and "SEQUENCE" properties match, the "DTSTAMP" property is used as the tie-breaker. The component with the latest "DTSTAMP" overrides all others. Similarly, for "Attendee" responses where the "UID" property values match and the "SEQUENCE" property values match, the response with the latest "DTSTAMP" overrides all others. 
Hence, CUAs must persist the following component properties: "UID", "RECURRENCE-ID", "SEQUENCE", and "DTSTAMP". Furthermore, for each "ATTENDEE" property of a component CUAs must persist the "SEQUENCE" and "DTSTAMP" property values associated with the "Attendee's" response.

\section{Application Protocol Elements}

ITIP messages are "text/calendar" MIME entities that contain calendaring and scheduling information. The particular type of [iCAL] message is referred to as the "method type". Each method type is identified by a "METHOD" property specified as part of the "text/calendar" content type. The table below shows various combinations of calendar components and the method types that this memo supports.

\begin{tabular}{|c|c|c|c|c|}
\hline & VEVENT & VTODO & VJOURNAL & VFREEBUSY \\
\hline \multicolumn{5}{|c|}{ 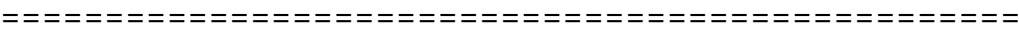 } \\
\hline Publish & Yes & Yes & Yes & Yes \\
\hline Request & Yes & Yes & No & Yes \\
\hline Refresh & Yes & Yes & No & No \\
\hline Cancel & Yes & Yes & Yes & No \\
\hline Add & Yes & Yes & Yes & No \\
\hline Reply & Yes & Yes & No & Yes \\
\hline $\begin{array}{l}\text { Counter } \\
\text { Decline- }\end{array}$ & Yes & Yes & No & No \\
\hline Counter & Yes & Yes & No & No \\
\hline
\end{tabular}

Each method type is defined in terms of its associated components and properties. Some components and properties are required, some are optional and others are excluded. The restrictions are expressed in this document using a simple "restriction table". The first column indicates the name of a component or property. Properties of the icalendar object are not indented. Properties of a component are indented. The second column contains "MUST" if the component or property must be present, "MAY" if the component or property is optional, and "NOT" if the component or property must not be present. Entries in the second column sometimes contain comments for further clarification. 


\subsection{Common Component Restriction Tables}

The restriction table below applies to properties of the icalendar object. That is, the properties at the outermost scope. The presence column uses the following values to assert whether a property is required, is optional and the number of times it may appear in the icalendar object.

\begin{tabular}{|c|c|}
\hline Presence Value & Description \\
\hline 1 & One instance MUST be present \\
\hline $1+$ & At least one instance MUST be present \\
\hline 0 & Instances of this property Must NOT be present \\
\hline $0+$ & Multiple instances MAY be present \\
\hline 0 or 1 & Up to 1 instance of this property MAY be present \\
\hline
\end{tabular}

The tables also call out "X-PROPERTY" and "X-COMPONENT" to show where vendor-specific properties and components can appear. The tables do not lay out the restrictions of property parameters. Those restrictions are defined in [iCAL].

Component/Property Presence

$\begin{array}{lll}\text { CALSCALE } & 0 \text { or } 1 & \\ \text { PRODID } & 1 & \\ \text { VERSION } & 1 & \\ \text { X-PROPERTY } & 0+ & \text { Value MUST be "2.0" }\end{array}$

DateTime values MAY refer to a "VTIMEZONE" component. The property restrictions in the table below apply to any "VTIMEZONE" component in an ITIP message.

Component/Property Presence

\begin{tabular}{|c|c|c|}
\hline VTIMEZONE & $0+$ & $\begin{array}{l}\text { MUST be present if any date/time refers } \\
\text { to timezone }\end{array}$ \\
\hline DAYLIGHT & $0+$ & $\begin{array}{l}\text { MUST be one or more of either STANDARD or } \\
\text { DAYLIGHT }\end{array}$ \\
\hline COMMENT & 0 or 1 & \\
\hline DTSTART & 1 & MUST be local time format \\
\hline RDATE & $0+$ & if present RRULE MUST NOT be present \\
\hline RRULE & $0+$ & if present RDATE MUST NOT be present \\
\hline TZNAME & 0 or 1 & \\
\hline TZOFFSET & 1 & \\
\hline TZOFFSETFROM & 1 & \\
\hline TZOFFSETTO & 1 & \\
\hline
\end{tabular}




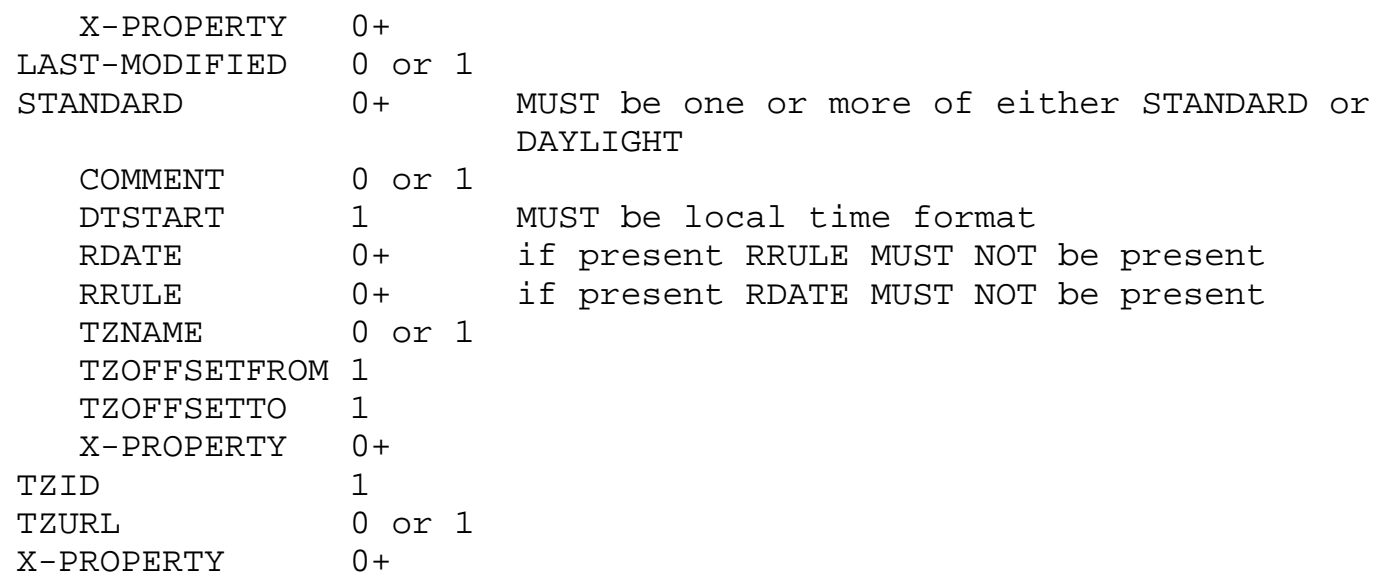

The property restrictions in the table below apply to any "VALARM" component in an ITIP message.

$\begin{array}{ll}\text { Component/Property } & \text { Presence } \\ \text { VALARM } & 0+ \\ \text { ACTION } & 1 \\ \text { ATTACH } & 0+ \\ \text { DESCRIPTION } & 0 \text { or } 1 \\ \text { DURATION } & 0 \text { or } 1 \text { if present REPEAT MUST be present } \\ \text { REPEAT } & 0 \text { or } 1 \text { if present DURATION MUST be present } \\ \text { SUMMARY } & 0 \text { or } 1 \\ \text { TRIGGER } & 1 \\ \text { X-PROPERTY } & 0+\end{array}$

3.2 Methods for VEVENT Calendar Components

This section defines the property set restrictions for the method types that are applicable to the "VEVENT" calendar component. Each method is defined using a table that clarifies the property constraints that define the particular method. 
The following summarizes the methods that are defined for the "VEVENT" calendar component.

\begin{tabular}{|c|c|}
\hline Method & Description \\
\hline \multicolumn{2}{|c|}{ 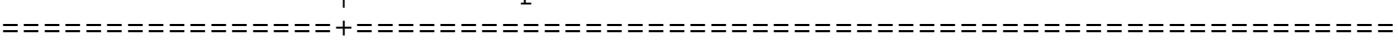 } \\
\hline PUBLISH & $\begin{array}{l}\text { Post notification of an event. Used primarily as } \\
\text { a method of advertising the existence of an } \\
\text { event. }\end{array}$ \\
\hline REQUEST & $\begin{array}{l}\text { Make a request for an event. This is an explicit } \\
\text { invitation to one or more "Attendees". Event } \\
\text { Requests are also used to update or change an } \\
\text { existing event. Clients that cannot handle } \\
\text { REQUEST may degrade the event to view it as an } \\
\text { PUBLISH. }\end{array}$ \\
\hline REPLY & $\begin{array}{l}\text { Reply to an event request. Clients may set their } \\
\text { status ("partstat") to ACCEPTED, DECLINED, } \\
\text { TENTATIVE, or DELEGATED. }\end{array}$ \\
\hline $\mathrm{ADD}$ & Add one or more instances to an existing event. \\
\hline CANCEL & $\begin{array}{l}\text { Cancel one or more instances of an existing } \\
\text { event. }\end{array}$ \\
\hline REFRESH & $\begin{array}{l}\text { A request is sent to an "Organizer" by an } \\
\text { "Attendee" asking for the latest version of an } \\
\text { event to be resent to the requester. }\end{array}$ \\
\hline COUNTER & $\begin{array}{l}\text { Counter a REQUEST with an alternative proposal, } \\
\text { Sent by an "Attendee" to the "Organizer". }\end{array}$ \\
\hline DECLINECOUNTER & $\begin{array}{l}\text { Decline a counter proposal. Sent to an } \\
\text { "Attendee" by the "Organizer". }\end{array}$ \\
\hline
\end{tabular}

\subsection{PUBLISH}

The "PUBLISH" method in a "VEVENT" calendar component is an unsolicited posting of an iCalendar object. Any CU may add published components to their calendar. The "Organizer" MUST be present in a published iCalendar component. "Attendees" MUST NOT be present. Its expected usage is for encapsulating an arbitrary event as an iCalendar object. The "Organizer" may subsequently update (with another "PUBLISH" method), add instances to (with an "ADD" method), or cancel (with a "CANCEL" method) a previously published "VEVENT" calendar component. 
This method type is an iCalendar object that conforms to the following property constraints:

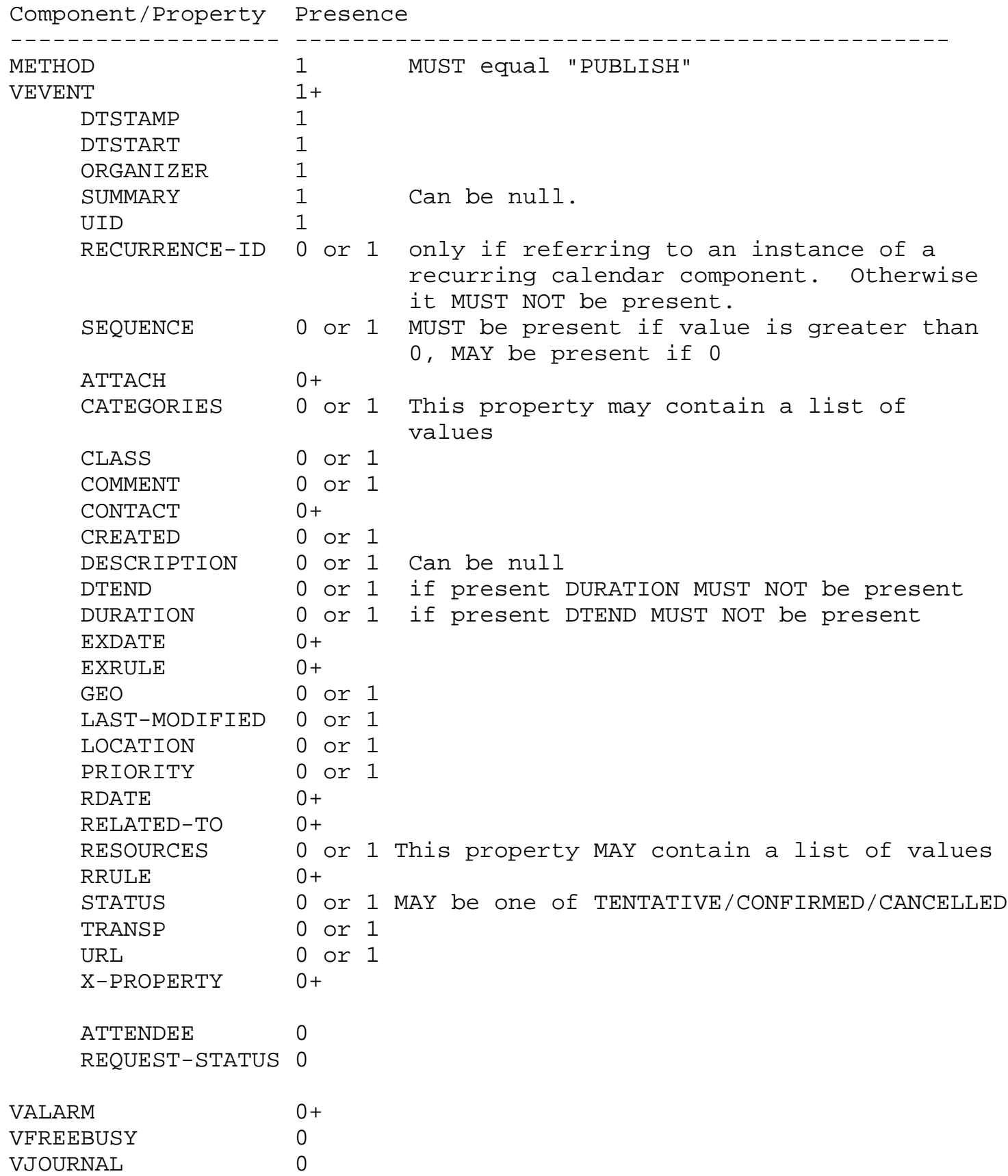

Silverberg, et. al. Standards Track [Page 16] 
VTODO

VTIMEZONE

$\mathrm{X}$-COMPONENT
0

$0+$

$0+$
MUST be present if any date/time refers to

a timezone

\section{2 .2 REQUEST}

The "REQUEST" method in a "VEVENT" component provides the following scheduling functions:

- Invite "Attendees" to an event;

- Reschedule an existing event;

- Response to a REFRESH request;

- Update the details of an existing event, without rescheduling it;

- Update the status of "Attendees" of an existing event, without rescheduling it;

- Reconfirm an existing event, without rescheduling it;

- Forward a "VEVENT" to another uninvited CU.

- For an existing "VEVENT" calendar component, delegate the role of "Attendee" to another $\mathrm{CU}$;

- For an existing "VEVENT" calendar component, changing the role of "Organizer" to another CU.

The "Organizer" originates the "REQUEST". The recipients of the "REQUEST" method are the CUs invited to the event, the "Attendees".

"Attendees" use the "REPLY" method to convey attendance status to the "Organizer".

The "UID" and "SEQUENCE" properties are used to distinguish the various uses of the "REQUEST" method. If the "UID" property value in the "REQUEST" is not found on the recipient's calendar, then the "REQUEST" is for a new "VEVENT" calendar component. If the "UID" property value is found on the recipient's calendar, then the "REQUEST" is for a rescheduling, an update, or a reconfirm of the "VEVENT" calendar component.

For the "REQUEST" method, multiple "VEVENT" components in a single iCalendar object are only permitted when for components with the same "UID" property. That is, a series of recurring events may have instance-specific information. In this case, multiple "VEVENT" components are needed to express the entire series. 
This method type is an icalendar object that conforms to the following property constraints:

Component/Property Presence

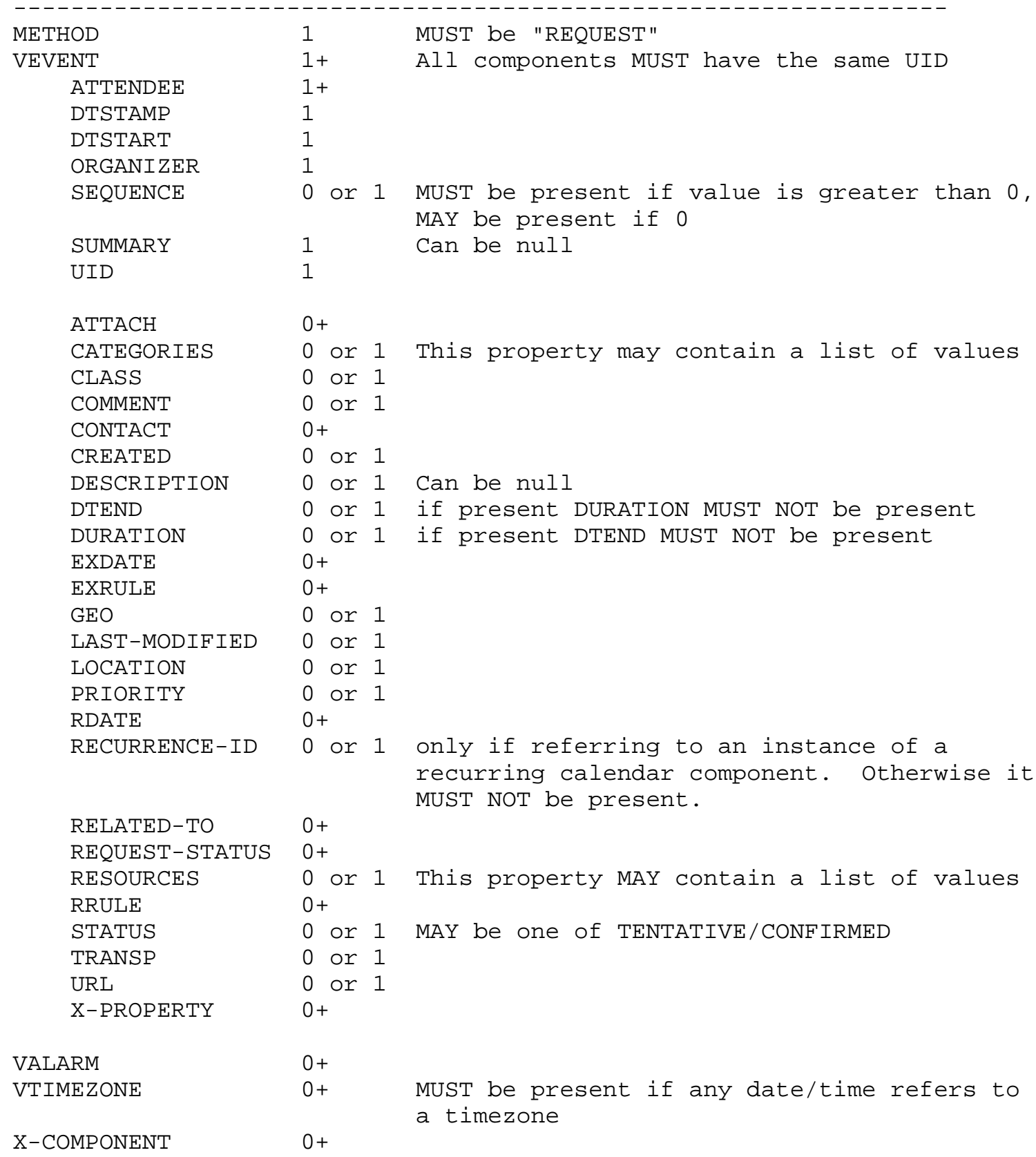


VFREEBUSY 0

VJOURNAL 0

VTODO

\subsubsection{Rescheduling an Event}

The "REQUEST" method may be used to reschedule an event. A rescheduled event involves a change to the existing event in terms of its time or recurrence intervals and possibly the location or description. If the recipient CUA of a "REQUEST" method finds that the "UID" property value already exists on the calendar, but that the "SEQUENCE" (or "DTSTAMP") property value in the "REQUEST" method is greater than the value for the existing event, then the "REQUEST" method describes a rescheduling of the event.

\subsubsection{Updating or Reconfirmation of an Event}

The "REQUEST" method may be used to update or reconfirm an event. An update to an existing event does not involve changes to the time or recurrence intervals, and might not involve a change to the location or description for the event. If the recipient CUA of a "REQUEST" method finds that the "UID" property value already exists on the calendar and that the "SEQUENCE" property value in the "REQUEST" is the same as the value for the existing event, then the "REQUEST" method describes an update of the event details, but no rescheduling of the event.

The update "REQUEST" method is the appropriate response to a "REFRESH" method sent from an "Attendee" to the "Organizer" of an event.

The "Organizer" of an event may also send unsolicited "REQUEST" methods. The unsolicited "REQUEST" methods may be used to update the details of the event without rescheduling it, to update the "partstat" parameter of "Attendees", or to reconfirm the event.

\subsubsection{Delegating an Event to another CU}

Some calendar and scheduling systems allow "Attendees" to delegate their presence at an event to another calendar user. ITIP supports this concept using the following workflow. Any "Attendee" may delegate their right to participate in a calendar VEVENT to another CU. The implication is that the delegate participates in lieu of the original "Attendee"; NOT in addition to the "Attendee". The delegator MUST notify the "Organizer" of this action using the steps outlined below. Implementations may support or restrict delegation as they see fit. For instance, some implementations may restrict a delegate from delegating a "REQUEST" to another CU. 
The "Delegator" of an event forwards the existing "REQUEST" to the "Delegate". The "REQUEST" method MUST include an "ATTENDEE" property with the calendar address of the "Delegate". The "Delegator" MUST also send a "REPLY" method to the "Organizer" with the "Delegator's" "ATTENDEE" property "partstat" parameter value set to "delegated". In addition, the "delegated-to" parameter MUST be included with the calendar address of the "Delegate".

In response to the request, the "Delegate" MUST send a "REPLY" method to the "Organizer" and optionally, to the "Delegator". The "REPLY" method " SHOULD include the "ATTENDEE" property with the "delegatedfrom" parameter value of the "Delegator's" calendar address.

The "Delegator" may continue to receive updates to the event even though they will not be attending. This is accomplished by the "Delegator" setting their "role" attribute to " NON-PARTICIPANT" in the "REPLY" to the "Organizer"

\subsubsection{Changing the Organizer}

The situation may arise where the "Organizer" of a VEVENT is no longer able to perform the "Organizer" role and abdicates without passing on the "Organizer" role to someone else. When this occurs the "Attendees" of the VEVENT may use out-of-band mechanisms to communicate the situation and agree upon a new "Organizer". The new "Organizer" should then send out a new "REQUEST" with a modified version of the VEVENT in which the "SEQUENCE" number has been incremented and value of the "ORGANIZER" property has been changed to the calendar address of the new "Organizer".

\subsubsection{Sending on Behalf of the Organizer}

There are a number of scenarios that support the need for a calendar user to act on behalf of the "Organizer" without explicit role changing. This might be the case if the CU designated as "Organizer" was sick or unable to perform duties associated with that function. In these cases iTIP supports the notion of one CU acting on behalf of another. Using the "sent-by" parameter, a calendar user could send an updated "VEVENT" REQUEST. In the case where one CU sends on behalf of another $\mathrm{CU}$, the "Attendee" responses are still directed back towards the CU designated as "Organizer".

\subsubsection{Forwarding to An Uninvited CU}

An "Attendee" invited to an event may invite another uninvited CU to the event. The invited "Attendee" accomplishes this by forwarding the original "REQUEST" method to the uninvited CU. The "Organizer" decides whether or not the uninvited CU is added to the attendee 
list. If the "Organizer" decides not to add the uninvited CU no further action is required, however the "Organizer" MAY send the uninvited CU a "CANCEL" message. If the "Organizer" decides to add an uninvited CU, a new "ATTENDEE" property is added for the uninvited CU with its property parameters set as the "Organizer" deems appropriate. When forwarding a "REQUEST" to another CU, the forwarding "Attendee" MUST NOT make changes to the VEVENT property set.

\subsubsection{Updating Attendee Status}

The "Organizer" of an event may also request updated status from one or more "Attendees. The "Organizer" sends a "REQUEST" method to the "Attendee" and sets the "ATTENDEE; RSVP=TRUE" property parameter. The "SEQUENCE" property for the event is not changed from its previous value. A recipient will determine that the only change in the "REQUEST" is that their "RSVP" property parameter indicates a request for updated status. The recipient SHOULD respond with a "REPLY" method indicating their current status with respect to the "REQUEST".

\section{2 .3 REPLY}

The "REPLY" method in a "VEVENT" calendar component is used to respond (e.g., accept or decline) to a "REQUEST" or to reply to a delegation "REQUEST". When used to provide a delegation response, the "Delegator" SHOULD include the calendar address of the "Delegate" on the "delegated-to" property parameter of the "Delegator's" "ATTENDEE" property. The "Delegate" SHould include the calendar address of the "Delegator" on the "delegated-from" property parameter of the "Delegate's" "ATTENDEE" property.

The "REPLY" method may also be used to respond to an unsuccessful "REQUEST" method. Depending on the value of the "REQUEST-STATUS" property no scheduling action may have been performed.

The "Organizer" of an event may receive the "REPLY" method from a CU not in the original "REQUEST". For example, a "REPLY" may be received from a "Delegate" to an event. In addition, the "REPLY" method may be received from an unknown CU (a "Party Crasher"). This uninvited "Attendee" may be accepted, or the "Organizer" may cancel the event for the uninvited "Attendee" by sending a "CANCEL" method to the uninvited "Attendee".

An "Attendee" can include a message to the "Organizer" using the "COMMENT" property. For example, if the user indicates tentative acceptance and wants to let the "Organizer" know why, the reason can be expressed in the "COMMENT" property value. 
The "Organizer" may also receive a "REPLY" from one CU on behalf of another. Like the scenario enumerated above for the "Organizer", "Attendees" may have another CU respond on their behalf. This is done using the "sent-by" parameter.

The optional properties listed in the table below (those listed as "0+" or "0 or 1") MUST NOT be changed from those of the original request. If property changes are desired the COUNTER message must be used.

This method type is an icalendar object that conforms to the following property constraints:

\begin{tabular}{|c|c|c|c|}
\hline Component/Property & Prese & $\mathrm{nc}$ & \\
\hline METHOD & 1 & & MUST be "REPLY" \\
\hline VEVENT & $1+$ & & All components MUST have the same UID \\
\hline ATTENDEE & 1 & & $\begin{array}{l}\text { MUST be the address of the Attendee } \\
\text { replying. }\end{array}$ \\
\hline DTSTAMP & 1 & & \\
\hline ORGANIZER & 1 & & \\
\hline RECURRENCE-ID & or & 1 & $\begin{array}{l}\text { only if referring to an instance of a } \\
\text { recurring calendar component. Otherwise } \\
\text { it must NOT be present. }\end{array}$ \\
\hline UID & 1 & & MUST be the UID of the original REQUEST \\
\hline SEQUENCE & or & 1 & $\begin{array}{l}\text { MUST if non-zero, MUST be the sequence } \\
\text { number of the original REQUEST. MAY be } \\
\text { present if } 0 \text {. }\end{array}$ \\
\hline $\mathrm{ATTACH}$ & $0+$ & & \\
\hline CATEGORIES & 0 or & 1 & This property may contain a list of values \\
\hline CLASS & 0 or & 1 & \\
\hline COMMENT & 0 or & 1 & \\
\hline CONTACT & $0+$ & & \\
\hline CREATED & 0 or & 1 & \\
\hline DESCRIPTION & or & 1 & \\
\hline DTEND & or & 1 & if present DURATION MUST NOT be present \\
\hline DTSTART & or & 1 & \\
\hline DURATION & 0 or & 1 & if present DTEND MUST NOT be present \\
\hline EXDATE & $0+$ & & \\
\hline EXRULE & $0+$ & & \\
\hline GEO & 0 or & 1 & \\
\hline LAST-MODIFIED & 0 or & 1 & \\
\hline LOCATION & 0 or & 1 & \\
\hline PRIORITY & 0 or & 1 & \\
\hline RDATE & $0+$ & & \\
\hline RELATED-TO & $0+$ & & \\
\hline
\end{tabular}




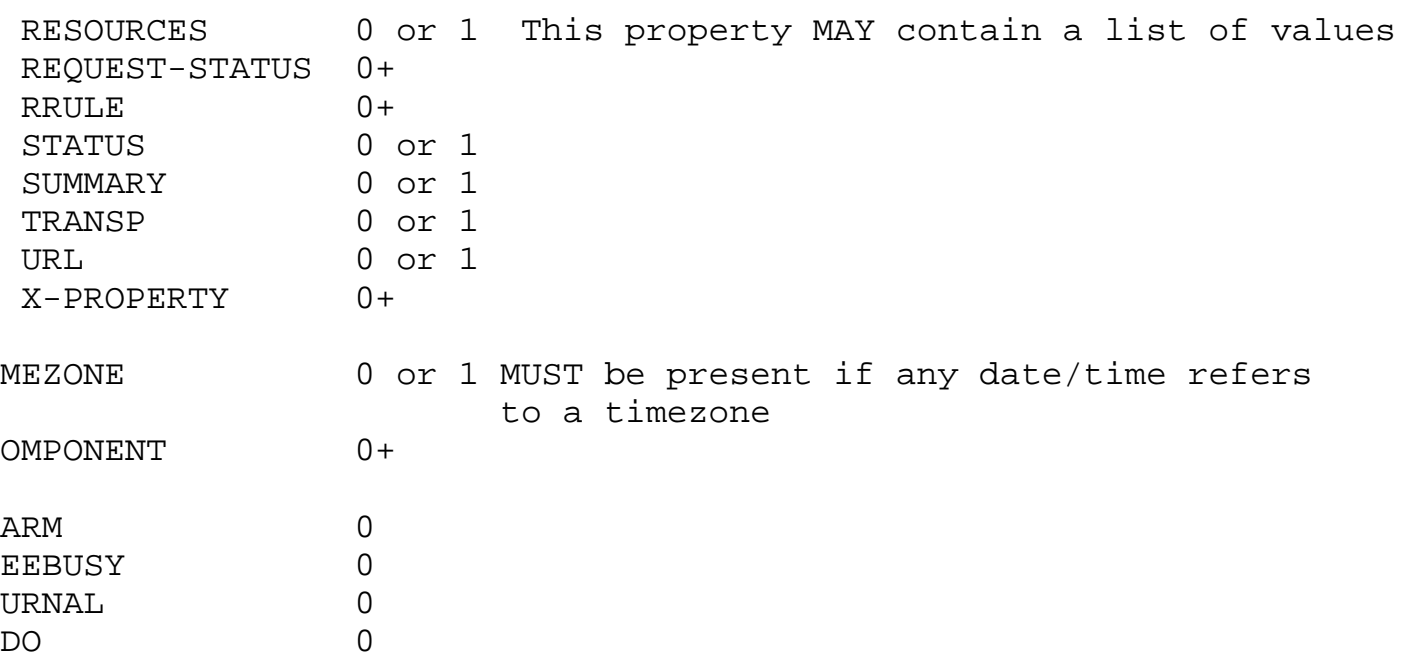

$3.2 .4 \mathrm{ADD}$

The "ADD" method in a "VEVENT" calendar component is used to add one or more instances to an existing "VEVENT". Unlike the "REQUEST" method, when using issuing an "ADD" method, the "Organizer" does not send the full "VEVENT" description; only the new instance(s). The "ADD" method is especially useful if there are instance-specific properties to be preserved in a recurring "VEVENT". For instance, an "Organizer" may have originally scheduled a weekly Thursday meeting. At some point, several instances changed. Location or start time may have changed, or some instances may have unique "DESCRIPTION" properties. The "ADD" method allows the "Organizer" to add new instances to an existing event using a single ITIP message without redefining the entire recurring pattern.

The "UID" must be that of the existing event. If the "UID" property value in the "ADD" is not found on the recipient's calendar, then the recipient SHOULD send a "REFRESH" to the "Organizer" in order to be updated with the latest version of the "VEVENT". If an "Attendee" implementation does not support the "ADD" method it should respond with a "REQUEST-STATUS" value of 3.14 and ask for a "REFRESH".

This method type is an icalendar object that conforms to the following property constraints: 


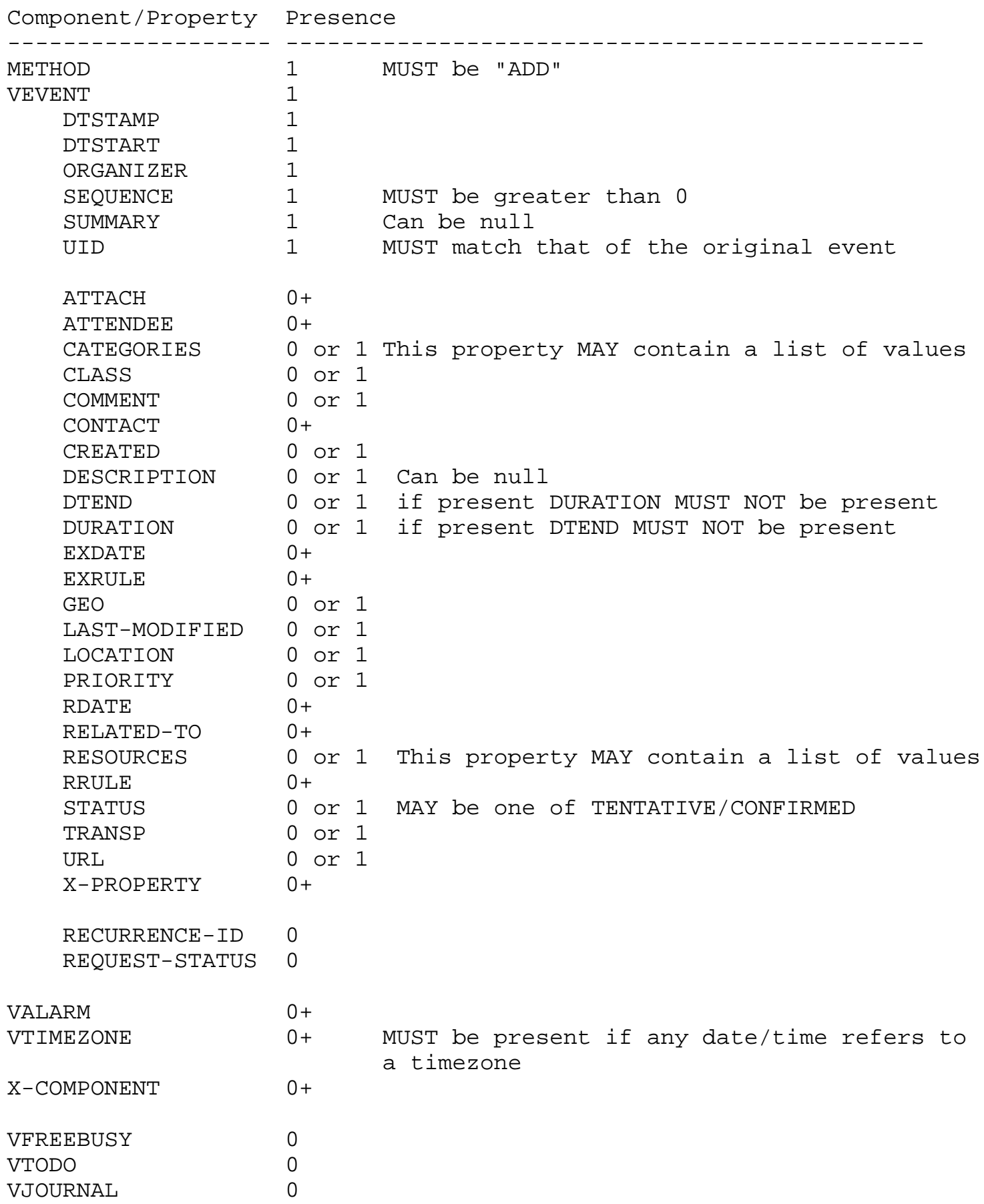




\subsubsection{CANCEL}

The "CANCEL" method in a "VEVENT" calendar component is used to send a cancellation notice of an existing event request to the

"Attendees". The message is sent by the "Organizer" of the event. For a recurring event, either the whole event or instances of an event may be cancelled. To cancel the complete range of recurring event, the "UID" property value for the event MUST be specified and a "RECURRENCE-ID" MUST NOT be specified in the "CANCEL" method. In order to cancel an individual instance of the event, the "RECURRENCE-ID" property value for the event MUST be specified in the "CANCEL" method.

There are two options for canceling a sequence of instances of a recurring "VEVENT" calendar component:

(a) the "RECURRENCE-ID" property for an instance in the sequence MUST be specified with the "RANGE" property parameter value of THISANDPRIOR (or THISANDFUTURE) to indicate cancellation of the specified "VEVENT" calendar component and all instances before (or after); or

(b) individual recurrence instances may be cancelled by specifying multiple "RECURRENCE-ID" properties corresponding to the instances to be cancelled.

When a "VEVENT" is cancelled, the "SEQUENCE" property value MUST be incremented.

This method type is an icalendar object that conforms to the following property constraints:

\begin{tabular}{|c|c|c|}
\hline Component/Property & Presenc & \\
\hline METHOD & 1 & MUST be "CANCEL" \\
\hline VEVENT & $1+$ & All must have the same UID \\
\hline ATTENDEE & $0+$ & $\begin{array}{l}\text { MUST include all "Attendees" being removed } \\
\text { the event. MUST include all "Attendees" if } \\
\text { the entire event is cancelled. }\end{array}$ \\
\hline DTSTAMP & 1 & \\
\hline ORGANIZER & 1 & \\
\hline SEQUENCE & 1 & \\
\hline UID & 1 & MUST be the UID of the original REQUEST \\
\hline COMMENT & 0 or 1 & \\
\hline ATTACH & $0+$ & \\
\hline CATEGORIES & 0 or 1 & This property may contain a list of \\
\hline
\end{tabular}




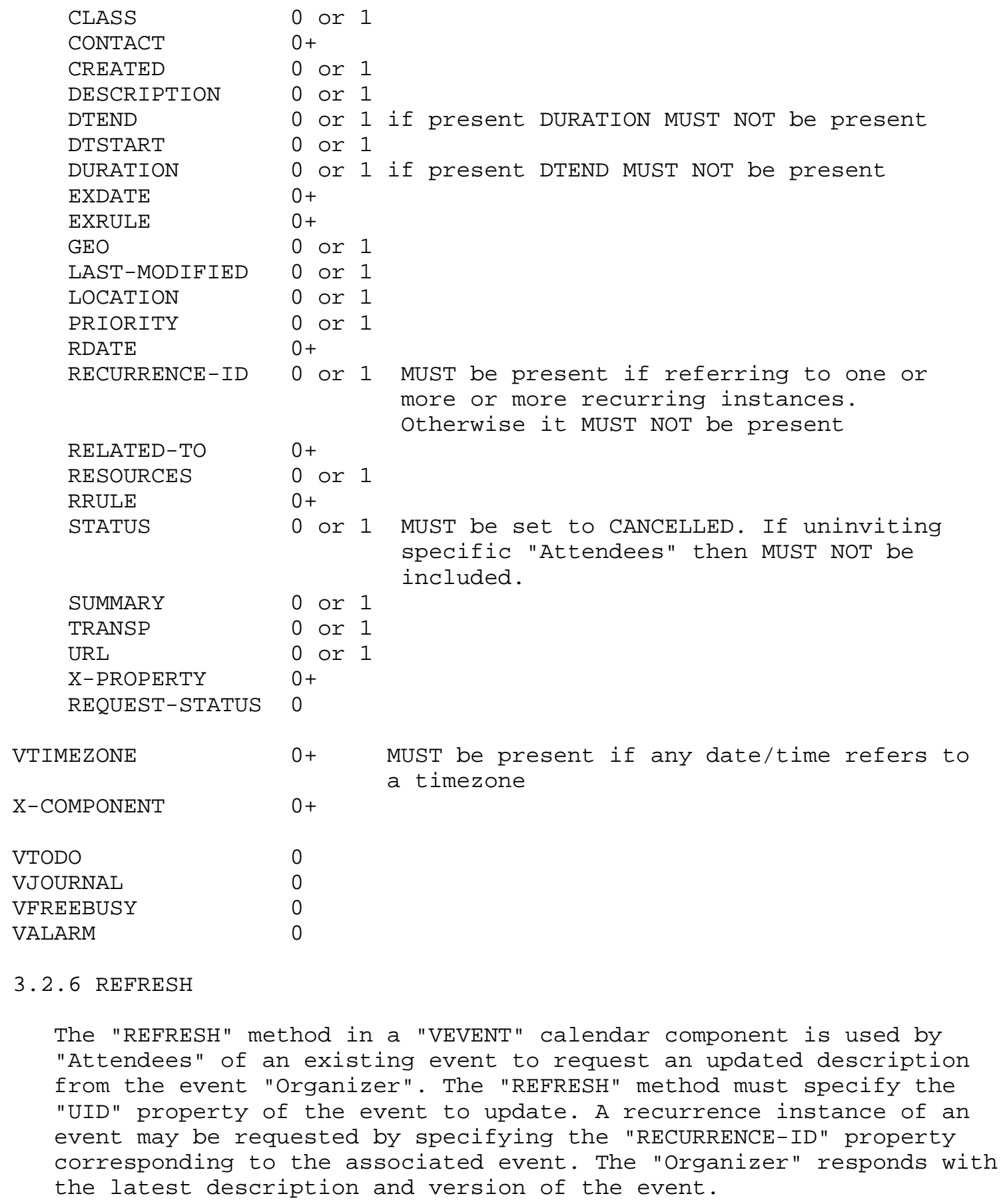

\subsubsection{REFRESH}

The "REFRESH" method in a "VEVENT" calendar component is used by "Attendees" of an existing event to request an updated description from the event "Organizer". The "REFRESH" method must specify the "UID" property of the event to update. A recurrence instance of an event may be requested by specifying the "RECURRENCE-ID" property corresponding to the associated event. The "Organizer" responds with the latest description and version of the event. 
This method type is an iCalendar object that conforms to the following property constraints:

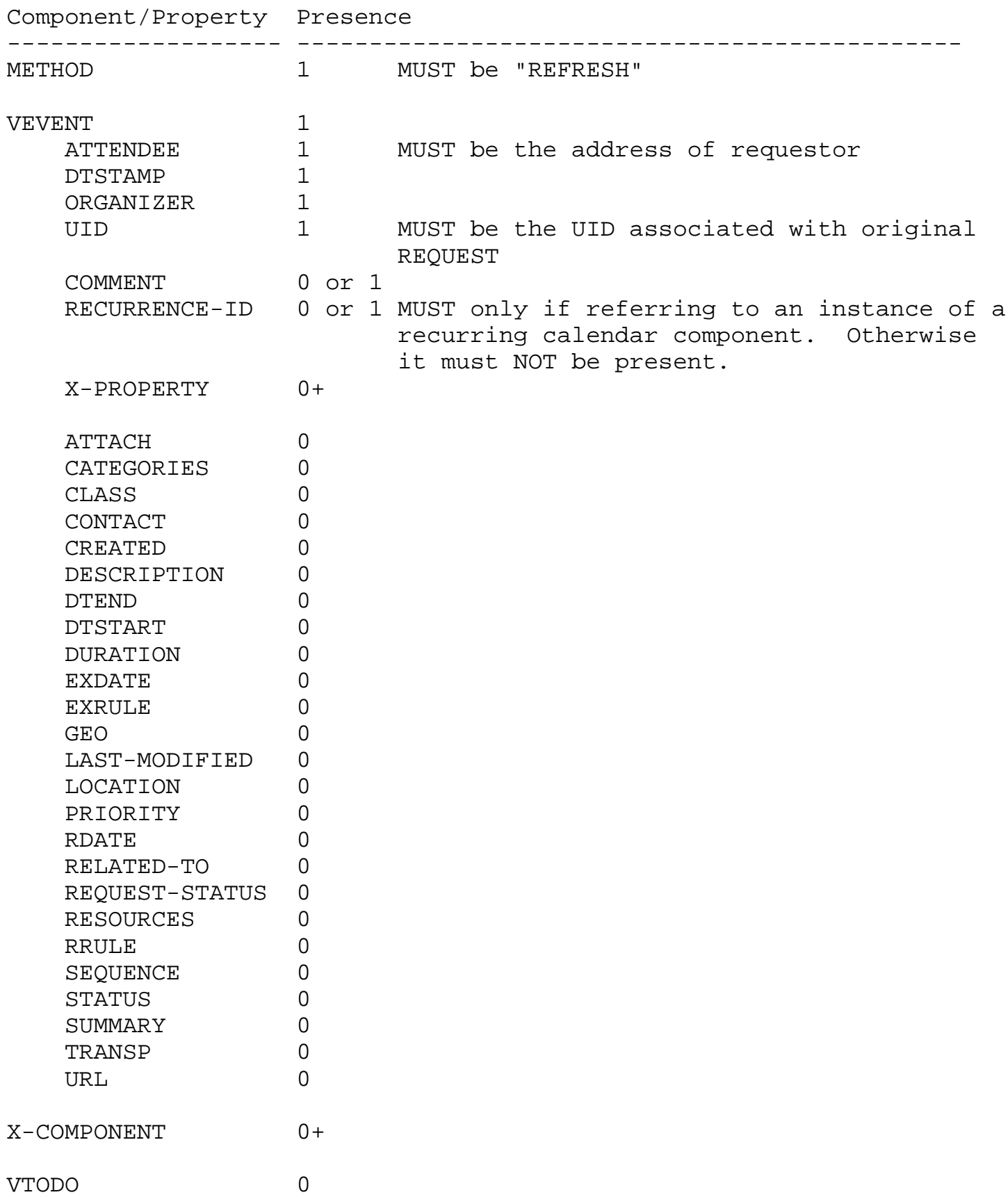

Silverberg, et. al. Standards Track [Page 27] 


$\begin{array}{ll}\text { VJOURNAL } & 0 \\ \text { VFREEBUSY } & 0 \\ \text { VTIMEZONE } & 0 \\ \text { VALARM } & 0\end{array}$

\section{2 .7 COUNTER}

The "COUNTER" method for a "VEVENT" calendar component is used by an "Attendee" of an existing event to submit to the "Organizer" a counter proposal to the event description. The "Attendee" sends this message to the "Organizer" of the event.

The counter proposal is an iCalendar object consisting of a VEVENT calendar component describing the complete description of the alternate event.

The "Organizer" rejects the counter proposal by sending the "Attendee" a VEVENT "DECLINECOUNTER" method. The "Organizer" accepts the counter proposal by rescheduling the event as described in section 3.2.2.1 Rescheduling an Event.

This method type is an iCalendar object that conforms to the following property constraints:

\begin{tabular}{|c|c|c|}
\hline Component/Property & Presenc & \\
\hline METHOD & 1 & MUST be "COUNTER" \\
\hline VEVENT & 1 & \\
\hline DTSTAMP & 1 & \\
\hline DTSTART & 1 & \\
\hline ORGANIZER & 1 & $\begin{array}{l}\text { MUST be the "Organizer" of the original } \\
\text { event }\end{array}$ \\
\hline SEQUENCE & 1 & $\begin{array}{l}\text { MUST be present if value is greater than } 0 \text {, } \\
\text { MAY be present if } 0\end{array}$ \\
\hline SUMMARY & 1 & Can be null \\
\hline UID & 1 & $\begin{array}{l}\text { MUST be the UID associated with the REQUEST } \\
\text { being countered }\end{array}$ \\
\hline ATTACH & $0+$ & \\
\hline ATTENDEE & $0+$ & $\begin{array}{l}\text { Can also be used to propose other } \\
\text { "Attendees" }\end{array}$ \\
\hline CATEGORIES & 0 or 1 & This property may contain a list of values \\
\hline CLASS & 0 or 1 & \\
\hline COMMENT & 0 or 1 & \\
\hline CONTACT & $0+$ & \\
\hline CREATED & 0 or 1 & \\
\hline DESCRIPTION & 0 or 1 & \\
\hline
\end{tabular}




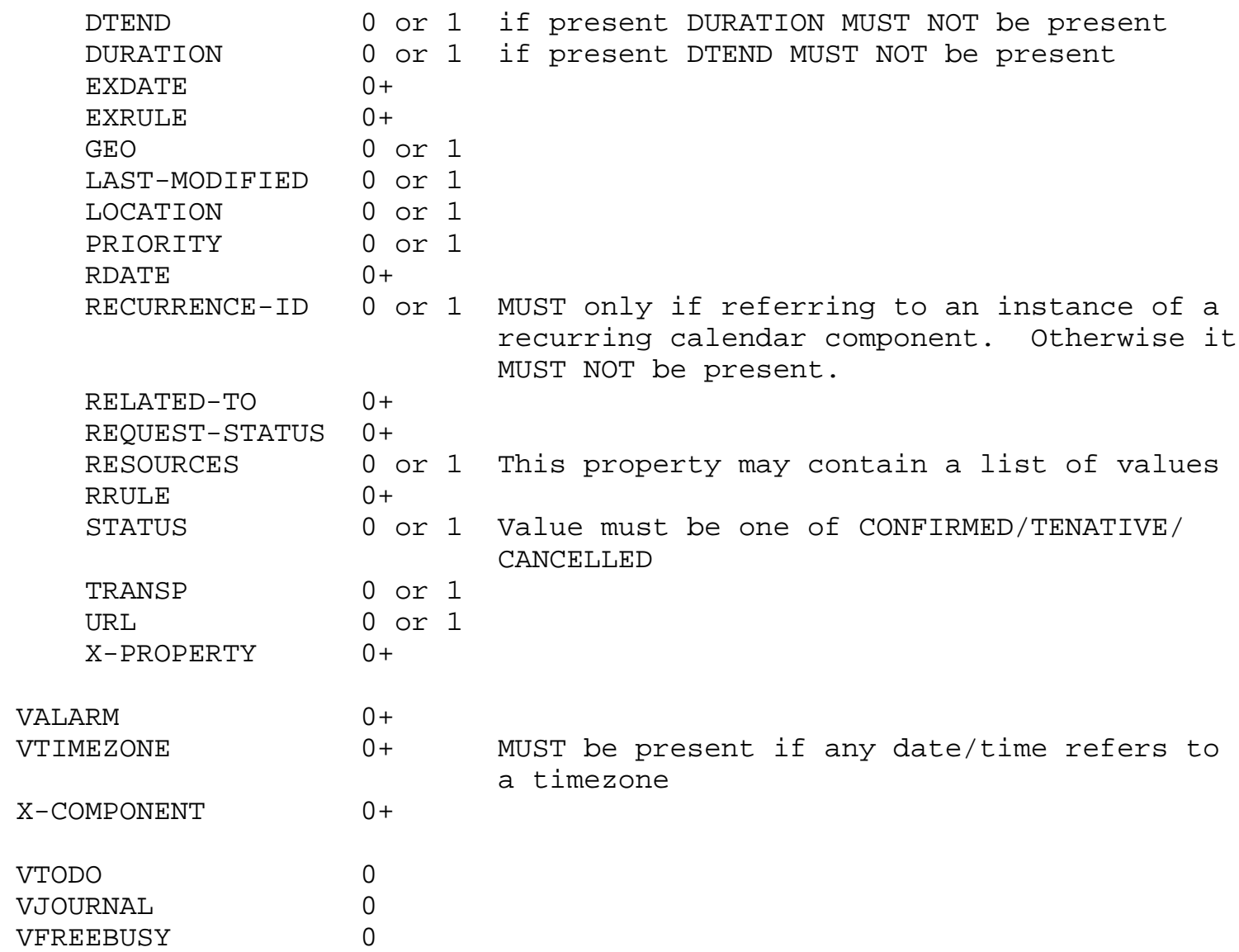

\subsubsection{DECLINECOUNTER}

The "DECLINECOUNTER" method in a "VEVENT" calendar component is used by the "Organizer" of an event to reject a counter proposal submitted by an "Attendee". The "Organizer" must send the "DECLINECOUNTER" message to the "Attendee" that sent the "COUNTER" method to the "Organizer".

This method type is an icalendar object that conforms to the following property constraints: 


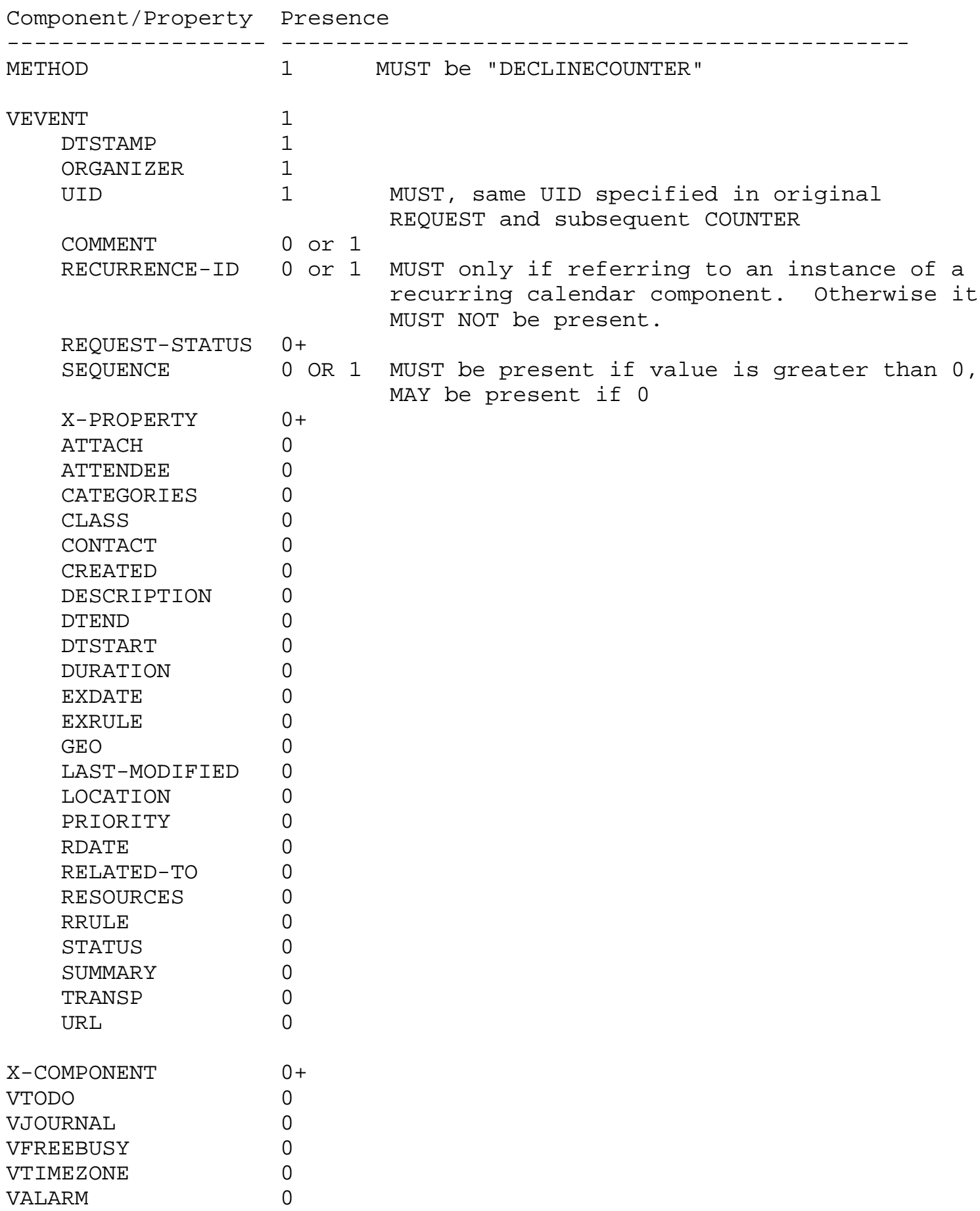

VALARM 0




\subsection{Methods For VFREEBUSY Components}

This section defines the property set for the methods that are applicable to the "VFREEBUSY" calendar component. Each of the methods is defined using a restriction table.

This document only addresses the transfer of busy time information. Applications desiring free time information MUST infer this from available busy time information.

The busy time information within the iCalendar object MAY be grouped into more than one "VFREEBUSY" calendar component. This capability allows busy time periods to be grouped according to some common periodicity, such as a calendar week, month, or year. In this case, each "VFREEBUSY" calendar component MUST include the "ATTENDEE", "DTSTART" and "DTEND" properties in order to specify the source of the busy time information and the date and time interval over which the busy time information covers.

The "FREEBUSY" property value MAY include a list of values, separated by the COMMA character ([US-ASCII] decimal 44). Alternately, multiple busy time periods MAY be specified with multiple instances of the "FREEBUSY" property. Both forms MUST be supported by implementations conforming to this document. Duplicate busy time periods SHOULD NOT be specified in an iCalendar object. However, two different busy time periods MAY overlap.

"FREEBUSY" properties should be sorted such that their values are in ascending order, based on the start time, and then the end time, with the earliest periods first. For example, today's busy time information should appear after yesterday's busy time information. And the busy time for this half-hour should appear after the busy time for earlier today.

Since events may span a day boundary, free busy time period may also span a day boundary. Individual "A" requests busy time from individuals "B", "C" and "D". Individual "B" and "C" replies with busy time data to individual "A". Individual "D" does not support busy time requests and does not reply with any data. If the transport binding supports exception messages, then individual "D" returns an "unsupported capability" message to individual "A4.34.3".

The following summarizes the methods that are defined for the "VFREEBUSY" calendar component. 


\begin{tabular}{|c|c|}
\hline 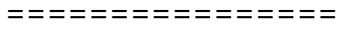 & :" \\
\hline Method & Description \\
\hline$===========$ & 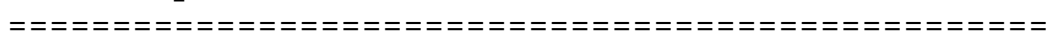 \\
\hline PUBLISH & Publish unsolicited busy time data. \\
\hline REQUEST & Request busy time data. \\
\hline REPLY & Reply to a busy time request. \\
\hline$=========$ & 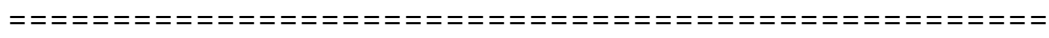 \\
\hline
\end{tabular}

\subsubsection{PUBLISH}

The "PUBLISH" method in a "VFREEBUSY" calendar component is used to publish busy time data. The method may be sent from one CU to any other. The purpose of the method is to provide a message for sending unsolicited busy time data. That is, the busy time data is not being sent as a "REPLY" to the receipt of a "REQUEST" method.

The "ATTENDEE" property must be specified in the busy time information. The value is the CU address of the originator of the busy time information.

This method type is an iCalendar object that conforms to the following property constraints:

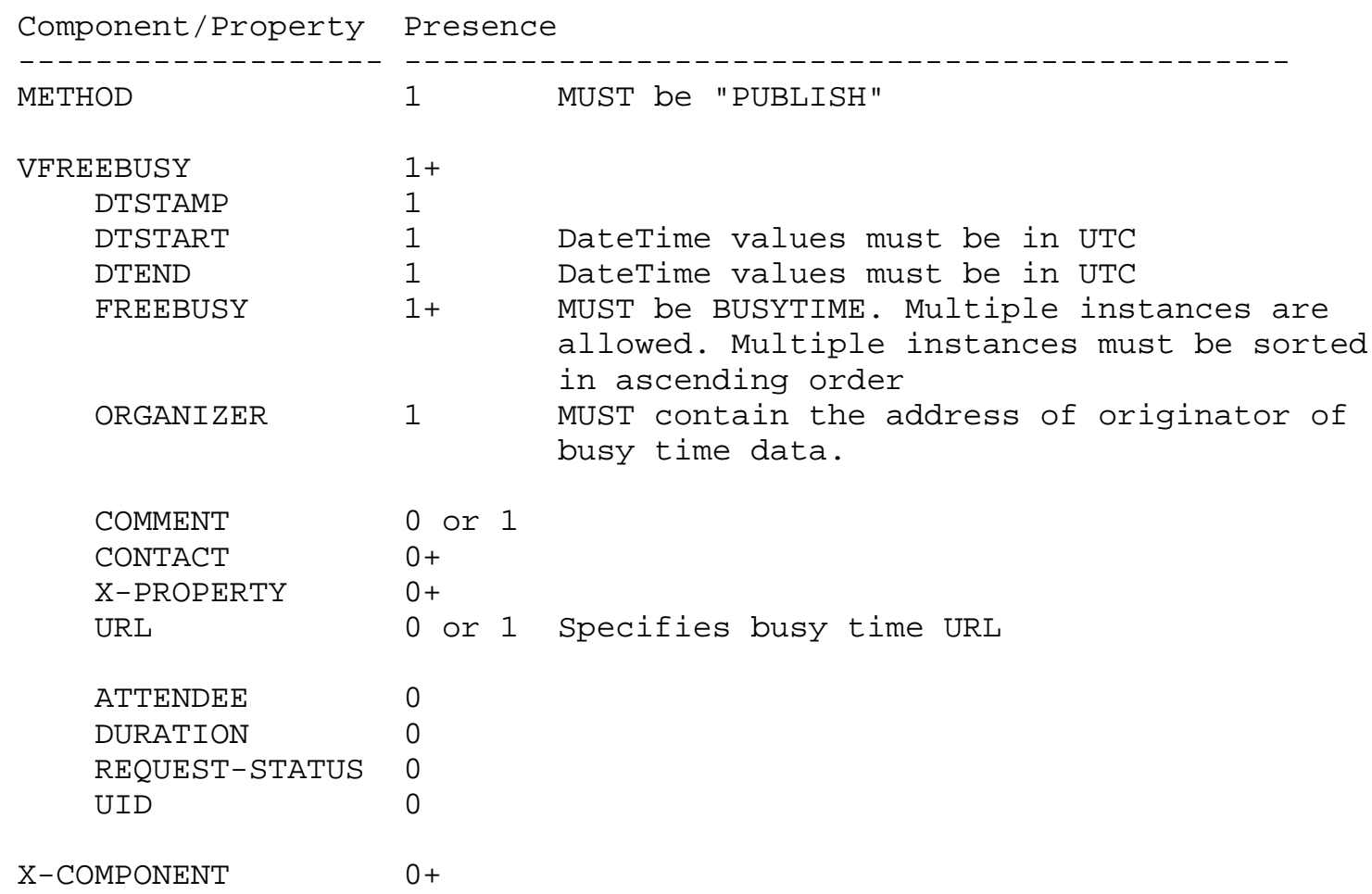




$\begin{array}{ll}\text { VEVENT } & 0 \\ \text { VTODO } & 0 \\ \text { VJOURNAL } & 0 \\ \text { VTIMEZONE } & 0 \\ \text { VALARM } & 0\end{array}$

\subsubsection{REQUEST}

The "REQUEST" method in a "VFREEBUSY" calendar component is used to ask a "Calendar User" for their busy time information. The request may be for a busy time information bounded by a specific date and time interval.

This message only permits requests for busy time information. The message is sent from a "Calendar User" requesting the busy time information to one or more intended recipients.

If the originator of the "REQUEST" method is not authorized to make a busy time request on the recipient's calendar system, then an exception message SHOULD be returned in a "REPLY" method, but no busy time data need be returned.

This method type is an icalendar object that conforms to the following property constraints:

\begin{tabular}{|c|c|c|}
\hline Component/Property & Presenc & \\
\hline METHOD & 1 & MUST be "REQUEST" \\
\hline VFREEBUSY & 1 & \\
\hline ATTENDEE & $1+$ & contain the address of the calendar store \\
\hline DTEND & 1 & DateTime values must be in UTC \\
\hline DTSTAMP & 1 & \\
\hline DTSTART & 1 & DateTime values must be in UTC \\
\hline ORGANIZER & 1 & MUST be the request originator's address \\
\hline UID & 1 & \\
\hline COMMENT & 0 or 1 & \\
\hline CONTACT & $0+$ & \\
\hline X-PROPERTY & $0+$ & \\
\hline FREEBUSY & 0 & \\
\hline DURATION & 0 & \\
\hline REQUEST-STATUS & 0 & \\
\hline URL & 0 & \\
\hline $\mathrm{X}-\mathrm{COMPONENT}$ & $0+$ & \\
\hline VALARM & 0 & \\
\hline VEVENT & 0 & \\
\hline
\end{tabular}




$\begin{array}{ll}\text { VTODO } & 0 \\ \text { VJOURNAL } & 0 \\ \text { VTIMEZONE } & 0\end{array}$

\subsubsection{REPLY}

The "REPLY" method in a "VFREEBUSY" calendar component is used to respond to a busy time request. The method is sent by the recipient of a busy time request to the originator of the request.

The "REPLY" method may also be used to respond to an unsuccessful "REQUEST" method. Depending on the "REQUEST-STATUS" value, no busy time information may be returned.

This method type is an icalendar object that conforms to the following property constraints:

\begin{tabular}{|c|c|c|}
\hline Component/Property & Presenc & \\
\hline METHOD & 1 & MUST be "REPLY" \\
\hline VFREEBUSY & 1 & \\
\hline ATTENDEE & 1 & (address of recipient replying) \\
\hline DTSTAMP & 1 & \\
\hline DTEND & 1 & DateTime values must be in UTC \\
\hline DTSTART & 1 & DateTime values must be in UTC \\
\hline FREEBUSY & $1+$ & $\begin{array}{l}\text { (values MUST all be of the same data } \\
\text { type. Multiple instances are allowed. } \\
\text { Multiple instances MUST be sorted in } \\
\text { ascending order. Values MAY NOT overlap) }\end{array}$ \\
\hline ORGANIZER & 1 & MUST be the request originator's address \\
\hline UID & 1 & \\
\hline COMMENT & 0 or 1 & \\
\hline CONTACT & $0+$ & \\
\hline REQUEST-STATUS & $0+$ & \\
\hline URL & 0 or 1 & (specifies busy time URL) \\
\hline X-PROPERTY & $0+$ & \\
\hline DURATION & 0 & \\
\hline SEQUENCE & 0 & \\
\hline $\mathrm{X}-\mathrm{COMPONENT}$ & $0+$ & \\
\hline VALARM & 0 & \\
\hline VEVENT & 0 & \\
\hline VTODO & 0 & \\
\hline VJOURNAL & 0 & \\
\hline VTIMEZONE & 0 & \\
\hline
\end{tabular}




\subsection{Methods For VTODO Components}

This section defines the property set for the methods that are applicable to the "VTODO" calendar component. Each of the methods is defined using a restriction table that specifies the property constraints that define the particular method.

The following summarizes the methods that are defined for the "VTODO" calendar component.

\begin{tabular}{|c|c|}
\hline Method & Description \\
\hline$==============$ & $=0==$ \\
\hline PUBLISH & $\begin{array}{l}\text { Post notification of a VTODO. Used primarily as } \\
\text { a method of advertising the existence of a VTODO. }\end{array}$ \\
\hline REQUEST & $\begin{array}{l}\text { Assign a VTODO. This is an explicit assignment to } \\
\text { one or more Calendar Users. The REQUEST method } \\
\text { is also used to update or change an existing } \\
\text { VTODO. Clients that cannot handle REQUEST MAY } \\
\text { degrade the method to treat it as a PUBLISH. }\end{array}$ \\
\hline REPLY & $\begin{array}{l}\text { Reply to a VTODO request. Attendees MAY set } \\
\text { PARTSTAT to ACCEPTED, DECLINED, TENTATIVE, } \\
\text { DELEGATED, PARTIAL, and COMPLETED. }\end{array}$ \\
\hline $\mathrm{ADD}$ & Add one or more instances to an existing to-do. \\
\hline CANCEL & $\begin{array}{l}\text { Cancel one or more instances of an existing } \\
\text { to-do. }\end{array}$ \\
\hline REFRESH & $\begin{array}{l}\text { A request sent to a VTODO Organizer asking for } \\
\text { the latest version of a VTODO. }\end{array}$ \\
\hline COUNTER & Counter a REQUEST with an alternative proposal. \\
\hline ECLIN & Decline a counter proposal by an Attendee. \\
\hline
\end{tabular}

\subsubsection{PUBLISH}

The "PUBLISH" method in a "VTODO" calendar component has no associated response. It is simply a posting of an iCalendar object that maybe added to a calendar. It MUST have an "Organizer". It MUST NOT have "Attendees". Its expected usage is for encapsulating an arbitrary "VTODO" calendar component as an iCalendar object. The "Organizer" MAY subsequently update (with another "PUBLISH" method), add instances to (with an "ADD" method), or cancel (with a "CANCEL" 


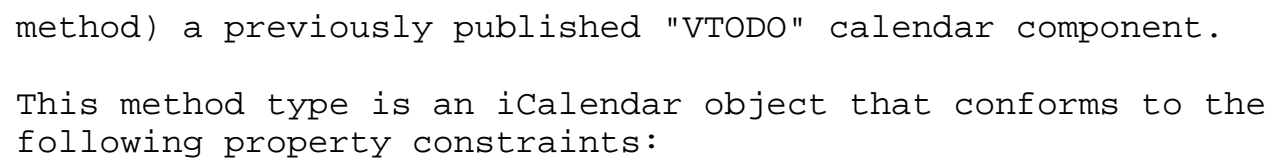




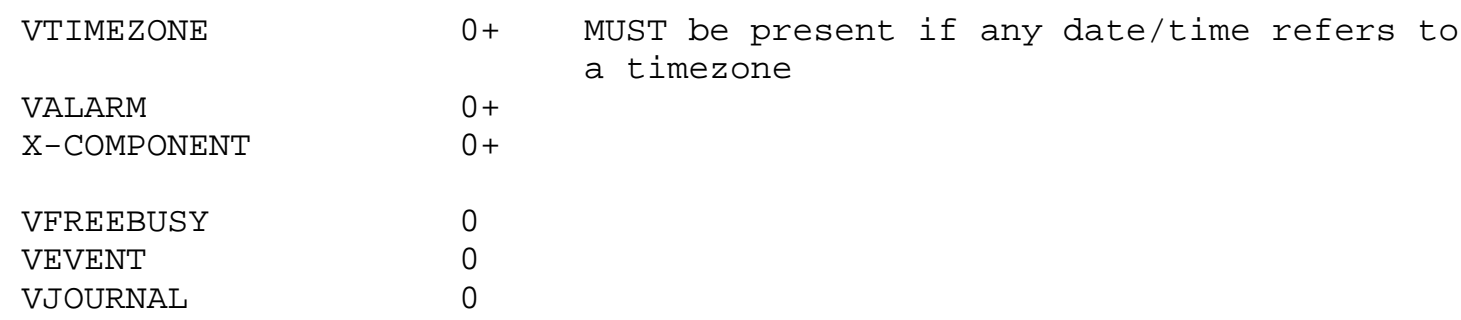

\subsubsection{REQUEST}

The "REQUEST" method in a "VTODO" calendar component provides the following scheduling functions:

- Assign a to-do to one or more "Calendar Users";

- Reschedule an existing to-do;

- Update the details of an existing to-do, without rescheduling it;

- Update the completion status of "Attendees" of an existing to-do, without rescheduling it;

- Reconfirm an existing to-do, without rescheduling it;

- Delegate/reassign an existing to-do to another "Calendar User".

The assigned "Calendar Users" are identified in the "VTODO" calendar component by individual "ATTENDEE; ROLE=REQ-PARTICIPANT" property value sequences.

The originator of a "REQUEST" is the "Organizer" of the to-do. The recipient of a "REQUEST" is the "Calendar User" assigned the to-do. The "Attendee" uses the "REPLY" method to convey their acceptance and completion status to the "Organizer" of the "REQUEST".

The "UID", "SEQUENCE", and "DTSTAMP" properties are used to distinguish the various uses of the "REQUEST" method. If the "UID" property value in the "REQUEST" is not found on the recipient's calendar, then the "REQUEST" is for a new to-do. If the "UID" property value is found on the recipient's calendar, then the "REQUEST" is a rescheduling, an update, or a reconfirm of the "VTODO" calendar object.

If the "Organizer" of the "REQUEST" method is not authorized to make a to-do request on the "Attendee's" calendar system, then an exception is returned in the "REQUEST-STATUS" property of a subsequent "REPLY" method, but no scheduling action is performed.

For the "REQUEST" method, multiple "VTODO" components in a single icalendar object are only permitted when for components with the same "UID" property. That is, a series of recurring events may have 


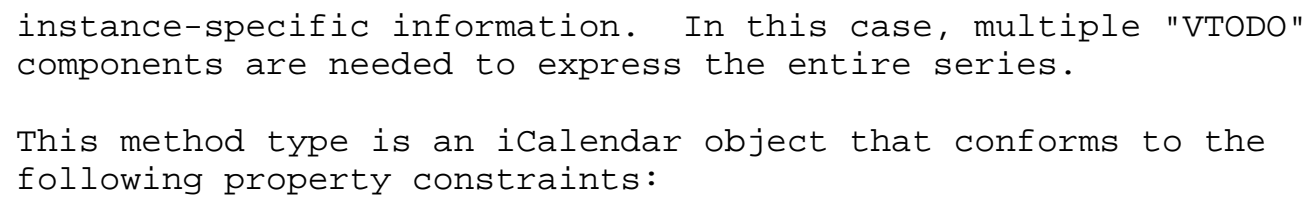




\begin{tabular}{ll}
\multicolumn{1}{l}{ REQUEST-STATUS } & 0 \\
VALARM & $0+$ \\
VTIMEZONE & $0+$ MUST be present if any date/time refers \\
X-COMPONENT & $0+$ \\
VEVENT & 0 \\
VEREEBUSY & 0 \\
VJOURNAL & 0
\end{tabular}

\subsubsection{REQUEST for Rescheduling a VTODO}

The "REQUEST" method may be used to reschedule a "VTODO" calendar component.

Rescheduling a "VTODO" calendar component involves a change to the existing "VTODO" calendar component in terms of its start or due time or recurrence intervals and possibly the description. If the recipient CUA of a "REQUEST" method finds that the "UID" property value already exists on the calendar, but that the "SEQUENCE" property value in the "REQUEST" is greater than the value for the existing VTODO, then the "REQUEST" method describes a rescheduling of the "VTODO" calendar component.

\subsubsection{REQUEST for Update or Reconfirmation of a VTODO}

The "REQUEST" method may be used to update or reconfirm a "VTODO" calendar component. Reconfirmation is merely an update of "Attendee" completion status or overall "VTODO" calendar component status.

An update to an existing "VTODO" calendar component does not involve changes to the start or due time or recurrence intervals, nor generally to the description for the "VTODO" calendar component. If the recipient CUA of a "REQUEST" method finds that the "UID" property value already exists on the calendar and that the "SEQUENCE" property value in the "REQUEST" is the same as the value for the existing event, then the "REQUEST" method describes an update of the "VTODO" calendar component details, but not a rescheduling of the "VTODO" calendar component.

The update "REQUEST" is the appropriate response to a "REFRESH" method sent from an "Attendee" to the "Organizer" of a "VTODO" calendar component.

Unsolicited "REQUEST" methods MAY be sent by the "Organizer" of a "VTODO" calendar component. The unsolicited "REQUEST" methods are 
used to update the details of the "VTODO" (without rescheduling it or updating the completion status of "Attendees") or the "VTODO" calendar component itself (i.e., reconfirm the "VTODO").

\subsubsection{REQUEST for Delegating a VTODO}

The "REQUEST" method is also used to delegate or reassign ownership of a "VTODO" calendar component to another "Calendar User". For example, it may be used to delegate an "Attendee's" role (i.e. "chair", or "participant") for a "VTODO" calendar component. The "REQUEST" method is sent by one of the "Attendees" of an existing

"VTODO" calendar component to some other individual. An "Attendee" of a "VTODO" calendar component MUST NOT delegate to the "Organizer" of the event.

For the purposes of this description, the "Attendee" delegating the "VTODO" calendar component is referred to as the "Delegator". The "Attendee" receiving the delegation request is referred to as the "Delegate".

The "Delegator" of a "VTODO" calendar component MUST forward the existing "REQUEST" method for a "VTODO" calendar component to the "Delegate". The "VTODO" calendar component description MUST include the "Delegator's" up-to-date "VTODO" calendar component definition. The "REQUEST" method MUST also include an "ATTENDEE" property with the calendar address of the "Delegate". The "Delegator" MUST also send a "REPLY" method back to the "Organizer" with the "Delegator's" "Attendee" property "partstat" parameter value set to "DELEGATED". In addition, the "delegated-to" parameter MUST be included with the calendar address of the "Delegate". A response to the delegation "REQUEST" is sent from the "Delegate" to the "Organizer" and optionally, to the "Delegator". The "REPLY" method from the "Delegate" SHOULD include the "ATTENDEE" property with their calendar address and the "delegated-from" parameter with the value of the "Delegator's" calendar address.

The delegation "REQUEST" method MUST assign a value for the "RSVP" property parameter associated with the "Delegator's" "Attendee" property to that of the "Delegate's" "ATTENDEE" property. For example if the "Delegator's" "ATTENDEE" property specifies "RSVP=TRUE", then the "Delegate's" "ATTENDEE" property MUST specify "RSVP=TRUE".

\subsubsection{REQUEST Forwarded To An Uninvited Calendar User}

An "Attendee" assigned a "VTODO" calendar component may send the "VTODO" calendar component to another new CU, not previously associated with the "VTODO" calendar component. The current 
"Attendee" assigned the "VTODO" calendar component does this by forwarding the original "REQUEST" method to the new CU. The new CU can send a "REPLY" to the "Organizer" of the "VTODO" calendar component. The reply contains an "ATTENDEE" property for the new CU.

The "Organizer" ultimately decides whether or not the new CU becomes part of the to-do and is not obligated to do anything with a "REPLY" from a new (uninvited) CU. If the "Organizer" does not want the new CU to be part of the to-do, the new "ATTENDEE" property is not added to the "VTODO" calendar component. The "Organizer" MAY send the CU a "CANCEL" message to indicate that they will not be added to the todo. If the "Organizer" decides to add the new CU, the new "ATTENDEE" property is added to the "VTODO" calendar component. Furthermore, the "Organizer" is free to change any "ATTENDEE" property parameter from the values supplied by the new CU to something the "Organizer" considers appropriate.

\subsubsection{REQUEST Updated Attendee Status}

An "Organizer" of a "VTODO" may request an updated status from one or more "Attendees". The "Organizer" sends a "REQUEST" method to the "Attendee" with the "ATTENDEE;RSVP=TRUE" property sequence. The "SEQUENCE" property for the "VTODO" is not changed from its previous value. A recipient determines that the only change in the "REQUEST" is that their "RSVP" property parameter indicates a request for an updated status. The recipient SHOULD respond with a "REPLY" method indicating their current status with respect to the "REQUEST".

\section{4 .3 REPLY}

The "REPLY" method in a "VTODO" calendar component is used to respond (e.g., accept or decline) to a request or to reply to a delegation request. It is also used by an "Attendee" to update their completion status. When used to provide a delegation response, the "Delegator" MUST include the calendar address of the "Delegate" in the "delegated-to" parameter of the "Delegator's" "ATTENDEE" property. The "Delegate" MUST include the calendar address of the "Delegator" on the "delegated-from" parameter of the "Delegate's" "ATTENDEE" property.

The "REPLY" method MAY also be used to respond to an unsuccessful "VTODO" calendar component "REQUEST" method. Depending on the "REQUEST-STATUS" value, no scheduling action may have been performed.

The "Organizer" of a "VTODO" calendar component MAY receive a "REPLY" method from a "Calendar User" not in the original "REQUEST". For example, a "REPLY" method MAY be received from a "Delegate" of a "VTODO" calendar component. In addition, the "REPLY" method MAY be 
received from an unknown "Calendar User", having been forwarded the "REQUEST" by an original "Attendee" of the "VTODO" calendar component. This uninvited "Attendee" MAY be accepted, or the "Organizer" MAY cancel the "VTODO" calendar component for the uninvited "Attendee" by sending them a "CANCEL" method.

This method type is an iCalendar object that conforms to the following property constraints:

\begin{tabular}{|c|c|c|}
\hline Component/Property & Presen & \\
\hline METHOD & 1 & MUST be "REPLY" \\
\hline VTODO & $1+$ & All component MUST have the same UID \\
\hline ATTENDEE & $1+$ & \\
\hline DTSTAMP & 1 & \\
\hline ORGANIZER & 1 & \\
\hline REQUEST-STATUS & $1+$ & \\
\hline UID & 1 & $\begin{array}{l}\text { MUST must be the address of the replying } \\
\text { attendee }\end{array}$ \\
\hline $\mathrm{ATTACH}$ & $0+$ & \\
\hline CATEGORIES & 0 or 1 & This property may contain a list of values \\
\hline CLASS & 0 or 1 & \\
\hline COMMENT & 0 or 1 & \\
\hline CONTACT & $0+$ & \\
\hline CREATED & 0 or 1 & \\
\hline DESCRIPTION & 0 or 1 & \\
\hline DTSTART & 0 or 1 & \\
\hline DUE & 0 or 1 & If present DURATION MUST NOT be present \\
\hline DURATION & 0 or 1 & If present DUE MUST NOT be present \\
\hline EXDATE & $0+$ & \\
\hline EXRULE & $0+$ & \\
\hline GEO & 0 or 1 & \\
\hline LAST-MODIFIED & 0 or 1 & \\
\hline LOCATION & 0 or 1 & \\
\hline PERCENT-COMPLETE & 0 or 1 & \\
\hline PRIORITY & 0 or 1 & \\
\hline RDATE & $0+$ & \\
\hline RELATED-TO & $0+$ & \\
\hline RESOURCES & 0 or 1 & This property may contain a list of values \\
\hline RRULE & $0+$ & \\
\hline RECURRENCE-ID & 0 or 1 & $\begin{array}{l}\text { MUST only if referring to an instance of a } \\
\text { Recurring calendar component. Otherwise it } \\
\text { MUST NOT be present }\end{array}$ \\
\hline SEQUENCE & 0 or 1 & $\begin{array}{l}\text { MUST be the sequence number of } \\
\text { the original REQUEST if greater than } 0 . \\
\text { MAY be present if } 0 \text {. }\end{array}$ \\
\hline STATUS & 0 or 1 & \\
\hline
\end{tabular}




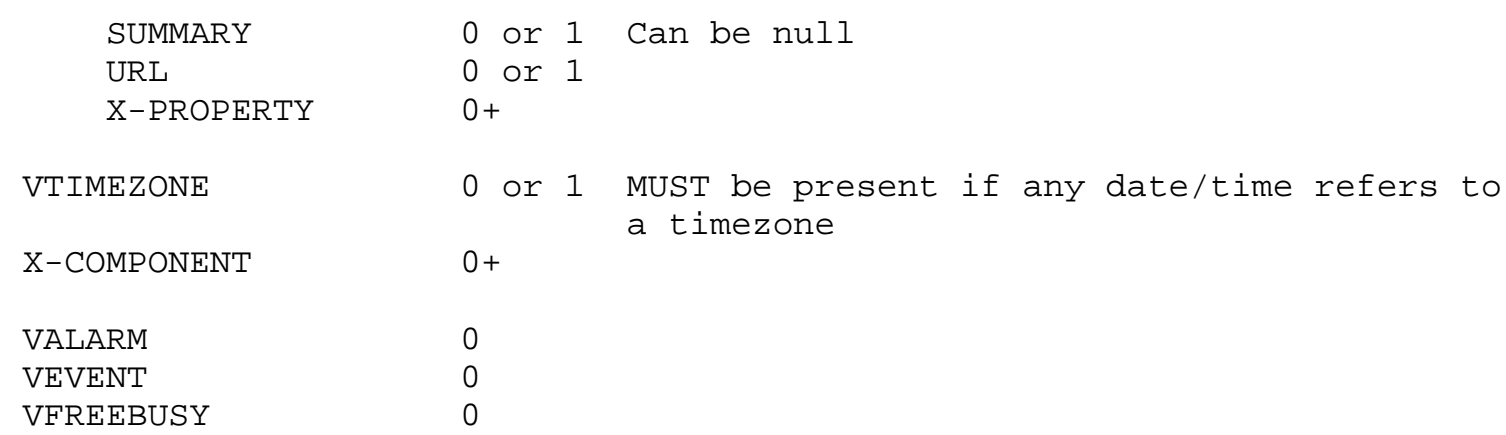

3.4.4 ADD

The "ADD" method in a "VTODO" calendar component is used to add one or more instances to an existing to-do.

If the "UID" property value in the "ADD" is not found on the recipient's calendar, then the recipient SHOULD send a "REFRESH" to the "Organizer" in order to be updated with the latest version of the "VTODO". If an "Attendee" implementation does not support the "ADD" method it should respond with a "REQUEST-STATUS" value of 5.3 and ask for a "REFRESH".

The "SEQUENCE" property value is incremented as the sequence of todos has changed.

This method type is an icalendar object that conforms to the following property constraints:

$\begin{array}{lcc}\text { Component/Property } & \text { Presence } & \\ - & 1 & \text { MUST be "ADD" } \\ \text { METHOD } & 1 & \\ \text { VTODO } & 1 & \\ \text { DTSTAMP } & 1 & \text { MUST be greater than } 0 \\ \text { ORGANIZER } & 1 & \text { Can be null. } \\ \text { PRIORITY } & 1 & \text { MUST match that of the original to-do } \\ \text { SEQUENCE } & 1 & \\ \text { SUMMARY } & 1 & \\ \text { UID } & 0+ & \\ \text { ATTACH } & 0+ & \\ \text { ATTENDEE } & 0 \text { or } 1 \text { This property may contain a list of } \\ \text { CATEGORIES } & 0 \text { or } 1 \text { values } \\ \text { CLASS } & 0 \text { or } 1\end{array}$

Silverberg, et. al. Standards Track [Page 43] 


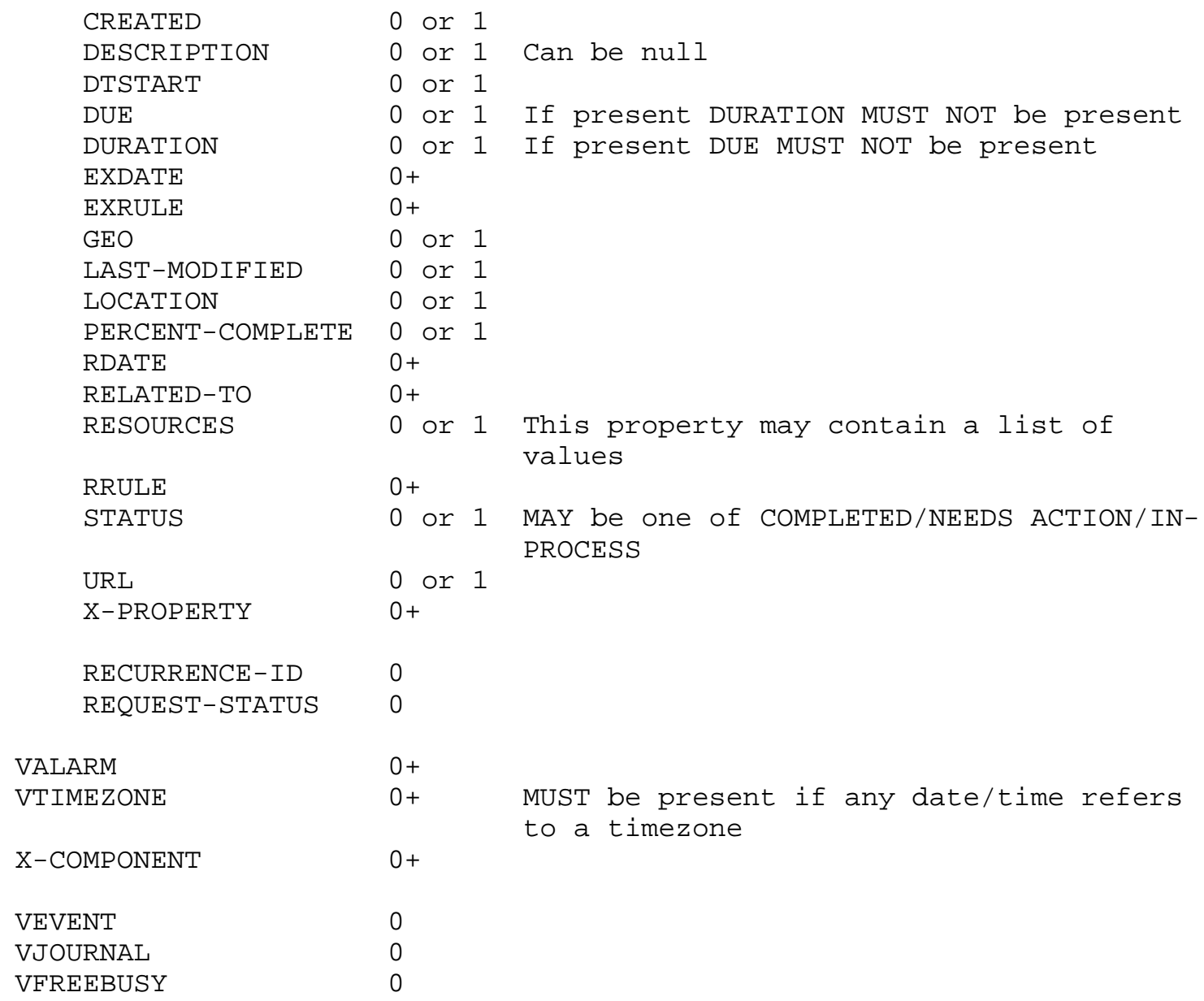

\section{4 .5 CANCEL}

The "CANCEL" method in a "VTODO" calendar component is used to send a cancellation notice of an existing "VTODO" calendar request to the "Attendees". The message is sent by the "Organizer" of a "VTODO" calendar component to the "Attendees" of the "VTODO" calendar component. For a recurring "VTODO" calendar component, either the whole "VTODO" calendar component or instances of a "VTODO" calendar component may be cancelled. To cancel the complete range of a recurring "VTODO" calendar component, the "UID" property value for the "VTODO" calendar component MUST be specified and a "RECURRENCEID" MUST NOT be specified in the "CANCEL" method. In order to cancel an individual instance of a recurring "VTODO" calendar component, the "RECURRENCE-ID" property value for the "VTODO" calendar component MUST be specified in the "CANCEL" method. 
There are two options for canceling a sequence of instances of a recurring "VTODO" calendar component:

(a) the "RECURRENCE-ID" property for an instance in the sequence MUST be specified with the "RANGE" property parameter value of THISANDPRIOR (or THISANDFUTURE) to indicate cancellation of the specified "VTODO" calendar component and all instances before (or after); or

(b) individual recurrence instances may be cancelled by specifying multiple "RECURRENCE-ID" properties corresponding to the instances to be cancelled.

When a "VTODO" is cancelled, the "SEQUENCE" property value MUST be incremented.

This method type is an icalendar object that conforms to the following property constraints:

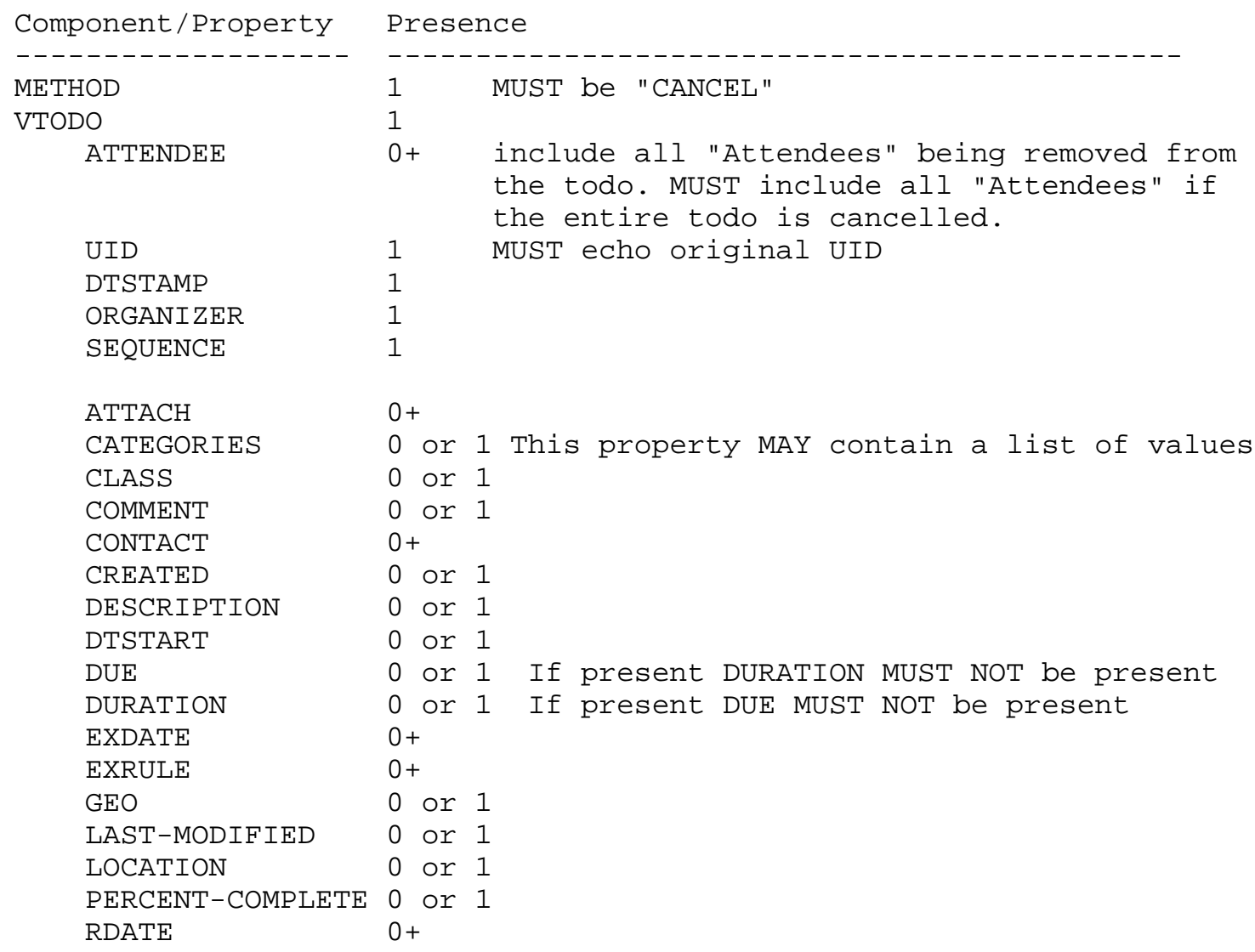




\begin{tabular}{|c|c|c|c|}
\hline RECURRENCE-ID & 0 or & 1 & $\begin{array}{l}\text { MUST only if referring to one or more } \\
\text { instances of a recurring calendar } \\
\text { component. Otherwise it MUST NOT be } \\
\text { present. }\end{array}$ \\
\hline RELATED-TO & $0+$ & & \\
\hline RESOURCES & 0 or & 1 & This property MAY contain a list of values \\
\hline RRULE & $0+$ & & \\
\hline PRIORITY & 0 or & 1 & \\
\hline STATUS & or & 1 & $\begin{array}{l}\text { If present it MUST be set to "CANCELLED". } \\
\text { MUST NOT be used if purpose is to remove } \\
\text { "ATTENDEES" rather than cancel the entire } \\
\text { VTODO. }\end{array}$ \\
\hline URL & 0 or & 1 & \\
\hline $\mathrm{X}-\mathrm{PROPERTY}$ & $0+$ & & \\
\hline REQUEST-STATUS & 0 & & \\
\hline VTIMEZONE & 0 or & 1 & $\begin{array}{l}\text { MUST be present if any date/time refers to } \\
\text { a timezone }\end{array}$ \\
\hline X-COMPONENT & $0+$ & & \\
\hline VALARM & 0 & & \\
\hline VEVENT & 0 & & \\
\hline VFREEBUSY & 0 & & \\
\hline
\end{tabular}

\section{4 .6 REFRESH}

The "REFRESH" method in a "VTODO" calendar component is used by "Attendees" of an existing "VTODO" calendar component to request an updated description from the "Organizer" of the "VTODO" calendar component. The "Organizer" of the "VTODO" calendar component MAY use this method to request an updated status from the "Attendees". The "REFRESH" method MUST specify the "UID" property corresponding to the "VTODO" calendar component needing update.

A refresh of a recurrence instance of a "VTODO" calendar component may be requested by specifying the "RECURRENCE-ID" property corresponding to the associated "VTODO" calendar component. The "Organizer" responds with the latest description and rendition of the "VTODO" calendar component. In most cases this will be a REQUEST unless the "VTODO" has been cancelled, in which case the ORGANIZER MUST send a "CANCEL". This method is intended to facilitate machine processing of requests for updates to a "VTODO" calendar component.

This method type is an icalendar object that conforms to the following property constraints: 


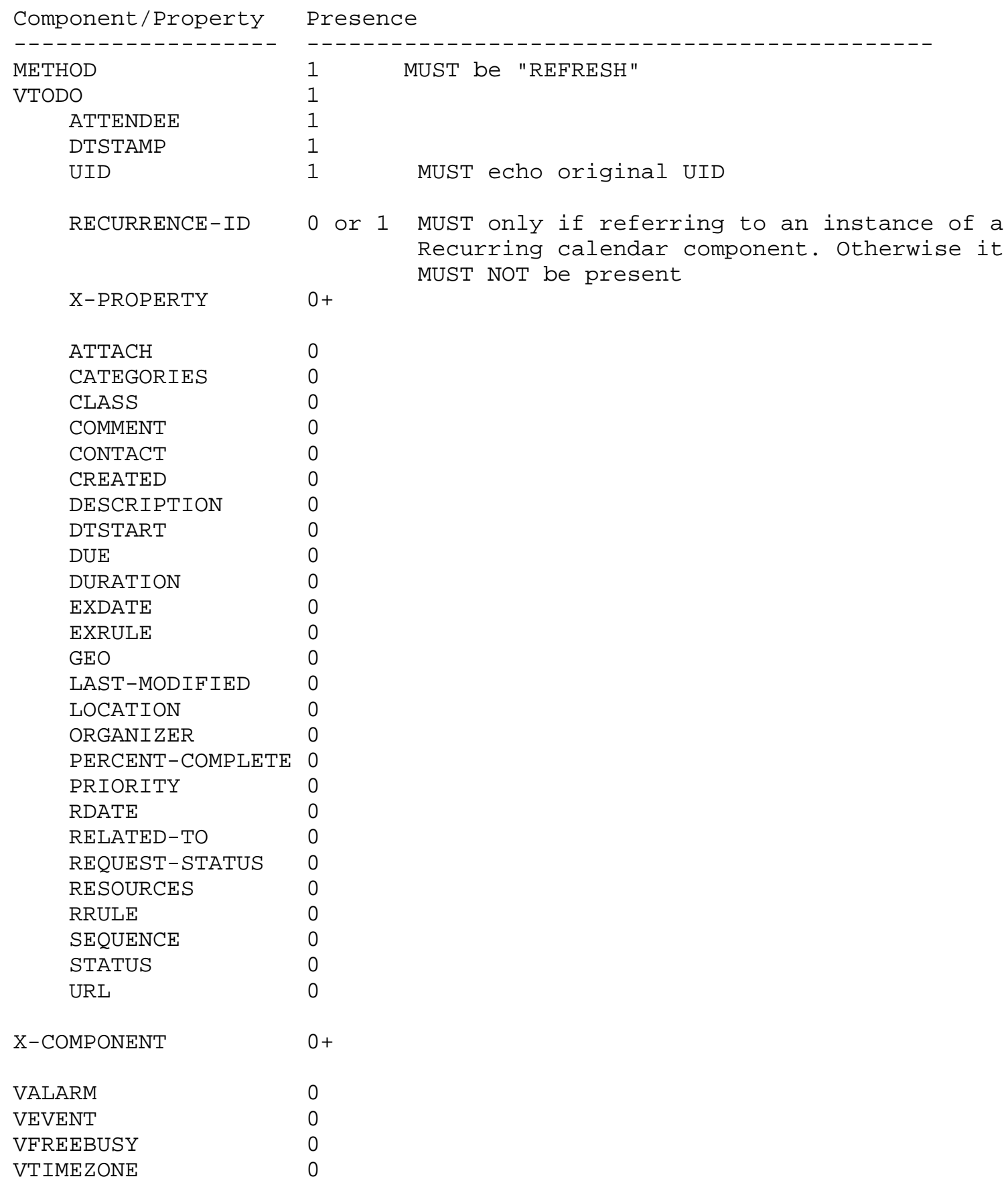

Silverberg, et. al. Standards Track [Page 47] 


\section{4 .7 COUNTER}

The "COUNTER" method in a "VTODO" calendar component is used by an "Attendee" of an existing "VTODO" calendar component to submit to the "Organizer" a counter proposal for the "VTODO" calendar component. The "Attendee" sends the message to the "Organizer" of the "VTODO" calendar component.

The counter proposal is an iCalendar object consisting of a "VTODO" calendar component describing the complete description of the alternate "VTODO" calendar component.

The "Organizer" rejects the counter proposal by sending the "Attendee" a "DECLINECOUNTER" method. The "Organizer" accepts the counter proposal by sending all of the "Attendees" of the "VTODO" calendar component a "REQUEST" method rescheduling the "VTODO" calendar component. In the latter case, the "Organizer" SHOULD reset the individual "RSVP" property parameter values to TRUE on each "ATTENDEE" property; in order to force a response by the "Attendees". This method type is an icalendar object that conforms to the following property constraints:

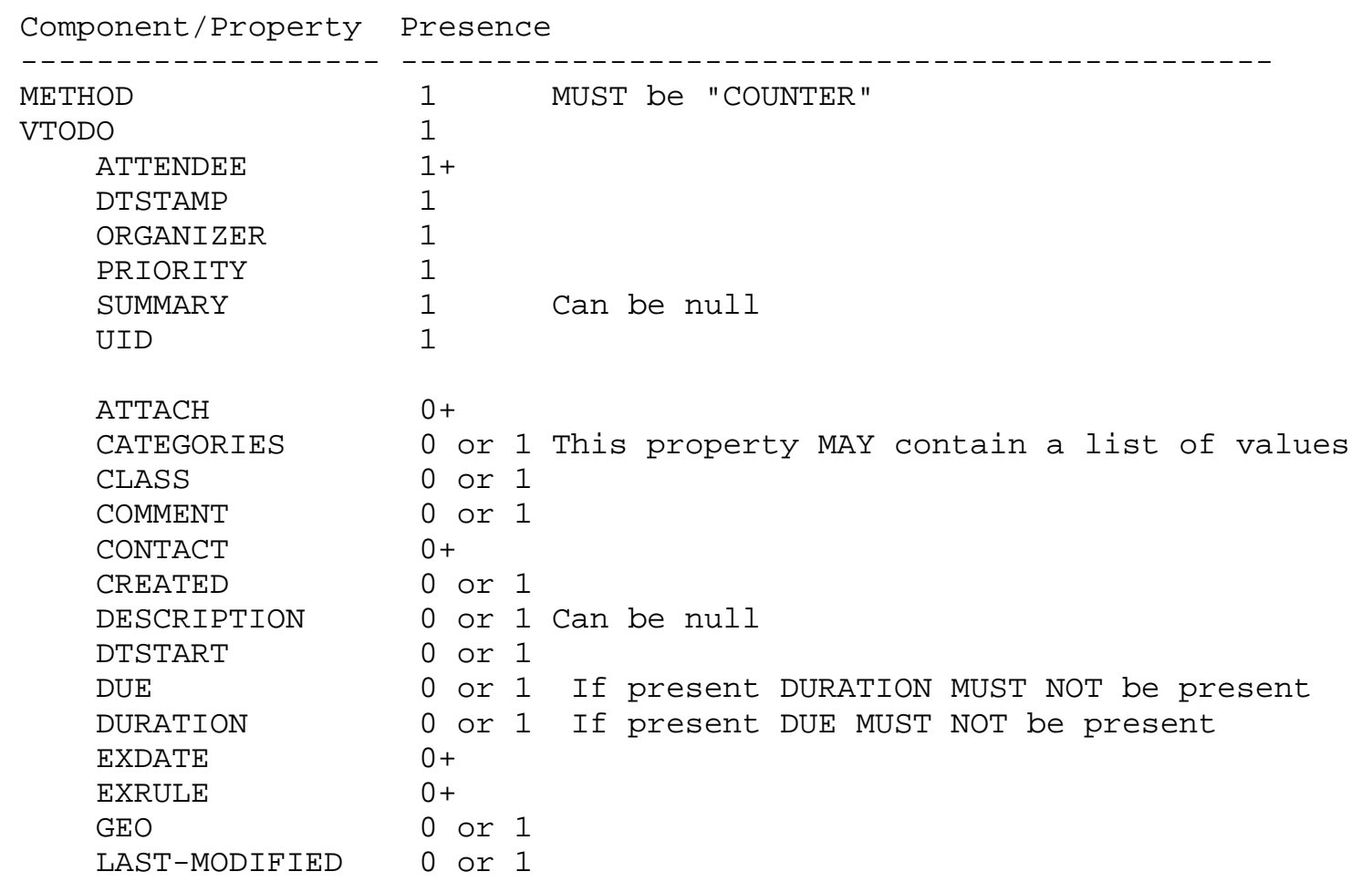




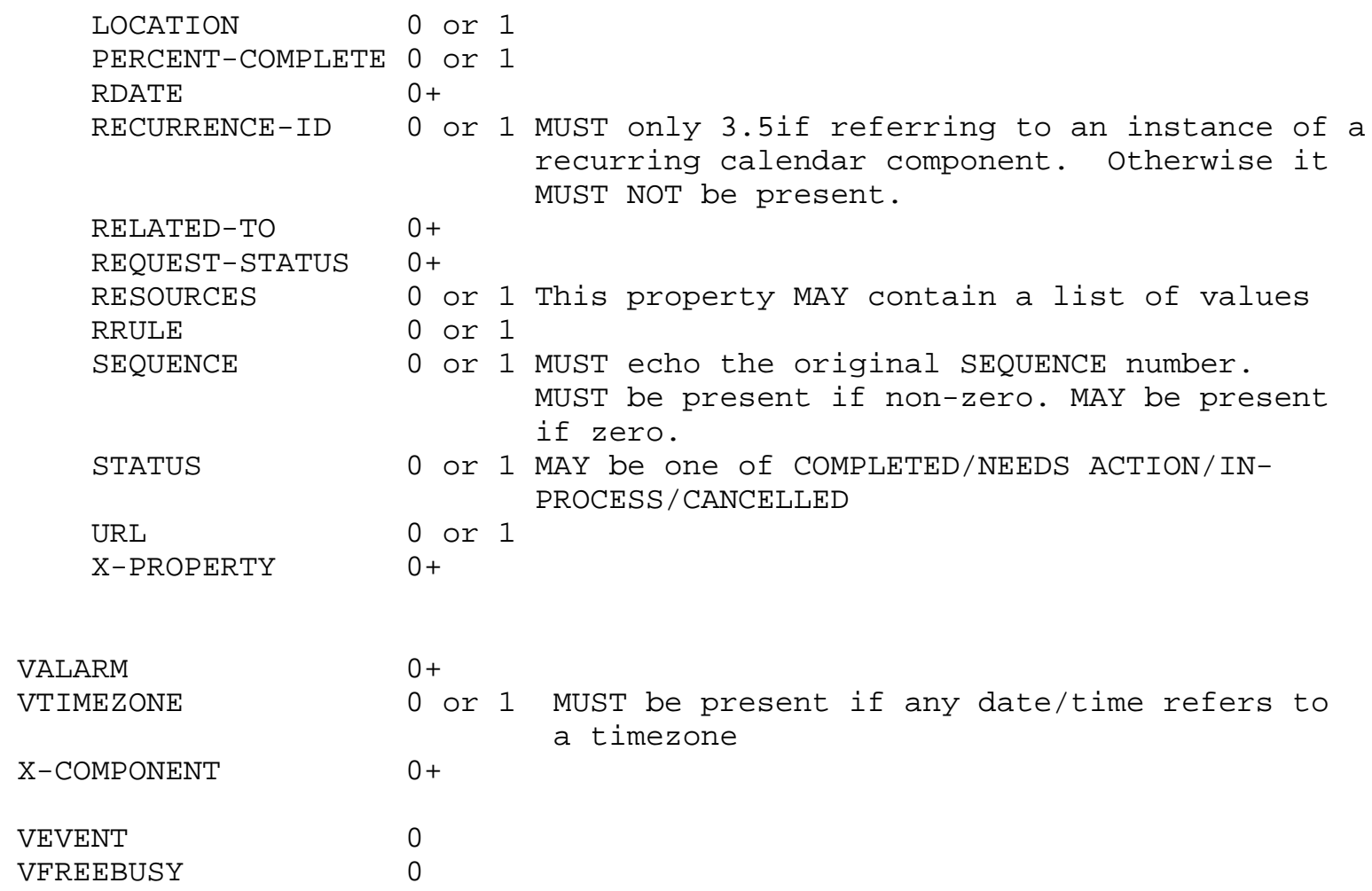

\subsubsection{DECLINECOUNTER}

The "DECLINECOUNTER" method in a "VTODO" calendar component is used by an "Organizer" of "VTODO" calendar component to reject a counter proposal offered by one of the "Attendees". The "Organizer" sends the message to the "Attendee" that sent the "COUNTER" method to the "Organizer".

This method type is an icalendar object that conforms to the following property constraints:

\begin{tabular}{|c|c|c|}
\hline Component / Property & \multicolumn{2}{|c|}{ Presence } \\
\hline METHOD & 1 & MUST be "DECLINECOUNTER" \\
\hline VTODO & 1 & \\
\hline ATTENDEE & $1+$ & MUST for all attendees \\
\hline DTSTAMP & 1 & \\
\hline ORGANIZER & 1 & \\
\hline SEQUENCE & 1 & MUST echo the original SEQUENCE number \\
\hline UID & 1 & MUST echo original UID \\
\hline
\end{tabular}

Silverberg, et. al. Standards Track [Page 49] 


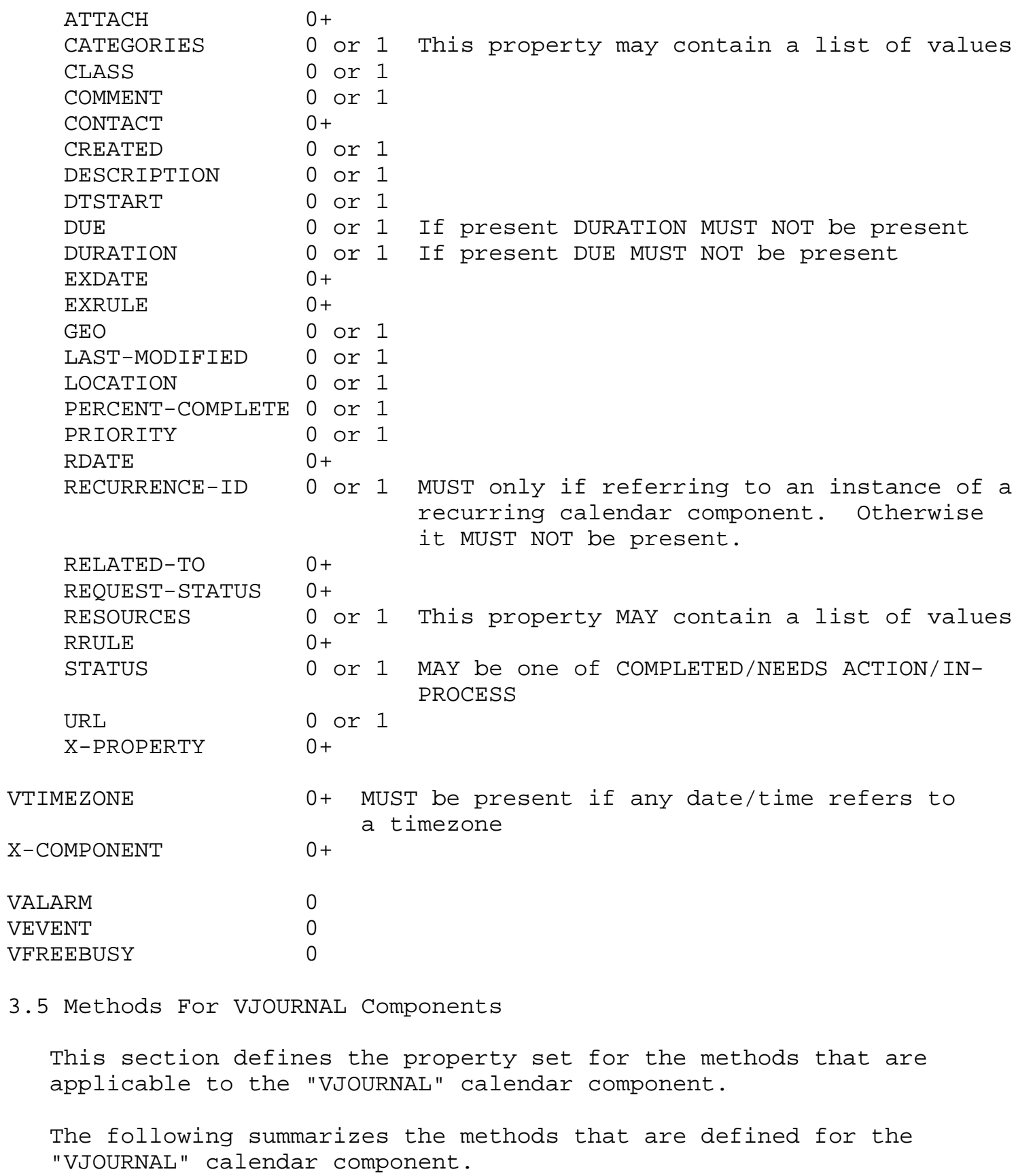

3. 5 Methods For VJOURNAL Components

This section defines the property set for the methods that are applicable to the "VJOURNAL" calendar component.

The following summarizes the methods that are defined for the "VJOURNAL" calendar component. 


\begin{tabular}{|c|c|}
\hline Method & Description \\
\hline \multicolumn{2}{|c|}{ =" =" } \\
\hline PUBLISH & $\begin{array}{l}\text { Post a journal entry. Used primarily as a method } \\
\text { of advertising the existence of a journal entry. }\end{array}$ \\
\hline $\mathrm{ADD}$ & $\begin{array}{l}\text { Add one or more instances to an existing journal } \\
\text { entry. }\end{array}$ \\
\hline CANCEL & $\begin{array}{l}\text { Cancel one or more instances of an existing } \\
\text { journal entry. }\end{array}$ \\
\hline
\end{tabular}

\subsubsection{PUBLISH}

The "PUBLISH" method in a "VJOURNAL" calendar component has no associated response. It is simply a posting of an icalendar object that may be added to a calendar. It MUST have an "Organizer". It MUST NOT have "Attendees". The expected usage is for encapsulating an

arbitrary journal entry as an iCalendar object. The "Organizer" MAY subsequently update (with another "PUBLISH" method) or cancel (with a "CANCEL" method) a previously published journal entry.

This method type is an icalendar object that conforms to the following property constraints:

\begin{tabular}{|c|c|c|}
\hline \multirow{2}{*}{ Component/Property } & \multicolumn{2}{|l|}{ Presence } \\
\hline & 1 & MUST be "PUBLISH" \\
\hline VJOURNAL & $1+$ & \\
\hline DESCRIPTION & 1 & Can be null. \\
\hline DTSTAMP & 1 & \\
\hline DTSTART & 1 & \\
\hline ORGANIZER & 1 & \\
\hline UID & 1 & \\
\hline ATTACH & $0+$ & \\
\hline CATEGORIES & 0 or 1 & This property MAY contain a list of values \\
\hline CLASS & 0 or 1 & \\
\hline COMMENT & 0 or 1 & \\
\hline CONTACT & $0+$ & \\
\hline CREATED & 0 or 1 & \\
\hline EXDATE & $0+$ & \\
\hline EXRULE & $0+$ & \\
\hline LAST-MODIFIED & 0 or 1 & \\
\hline RDATE & $0+$ & \\
\hline RECURRENCE-ID & 0 or 1 & MUST only if referring to an instance of a \\
\hline
\end{tabular}




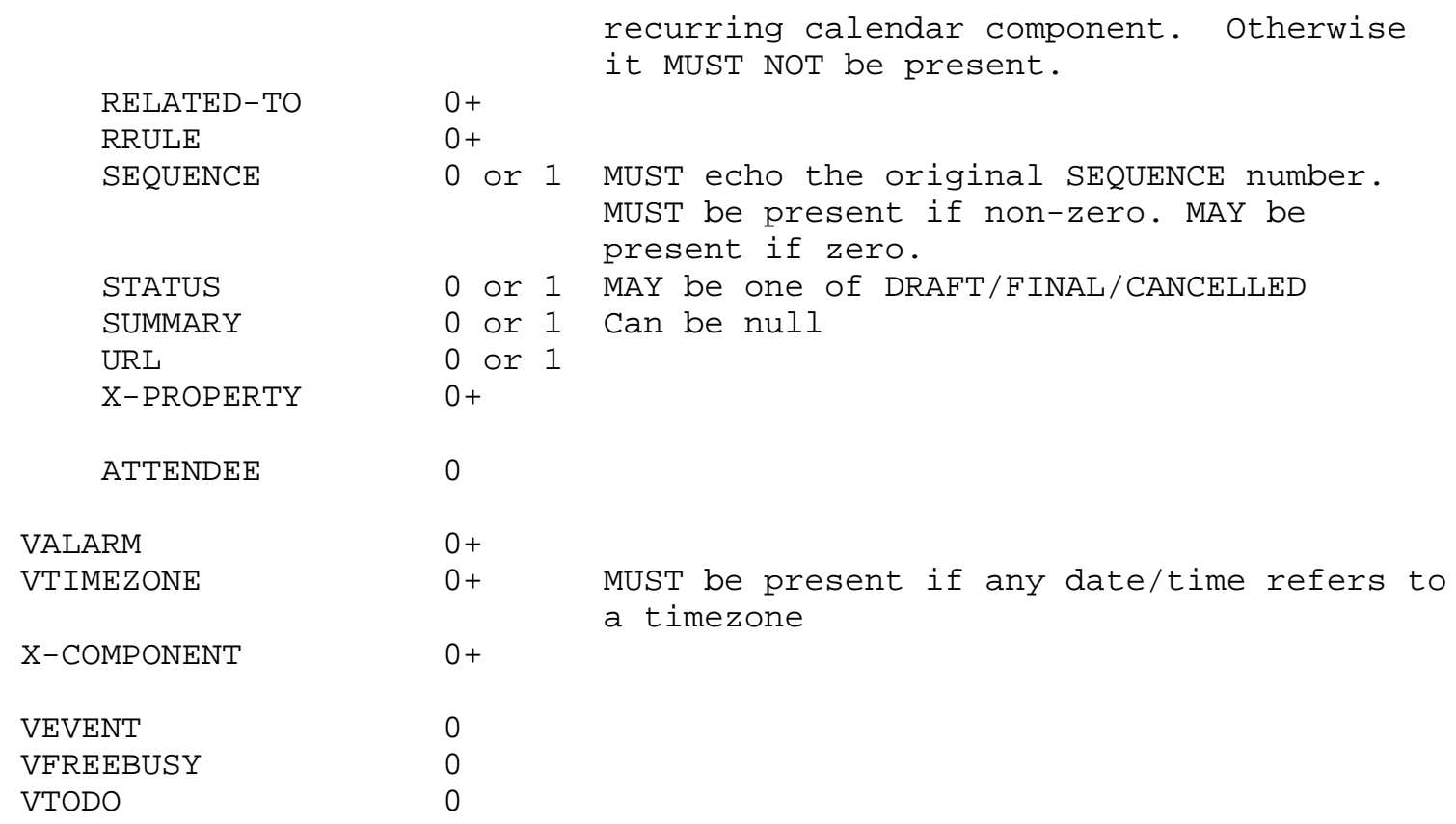

$3.5 .2 \mathrm{ADD}$

The "ADD" method in a "VJOURNAL" calendar component is used to add one or more instances to an existing "VJOURNAL" entry. There is no response to the "Organizer".

If the "UID" property value in the "ADD" is not found on the recipient's calendar, then the recipient MAY treat the "ADD" as a "PUBLISH".

This method type is an icalendar object that conforms to the following property constraints:

Component/Property Presence

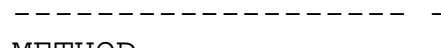

METHOD

VJOURNAL

DESCRIPTION

DTSTAMP

DTSTART

ORGANIZER

SEQUENCE

UID

ATTACH

Silverberg, et. al.
MUST be "ADD"

Can be null.

MUST be greater than 0

MUST match that of the original journal

$0+$ 


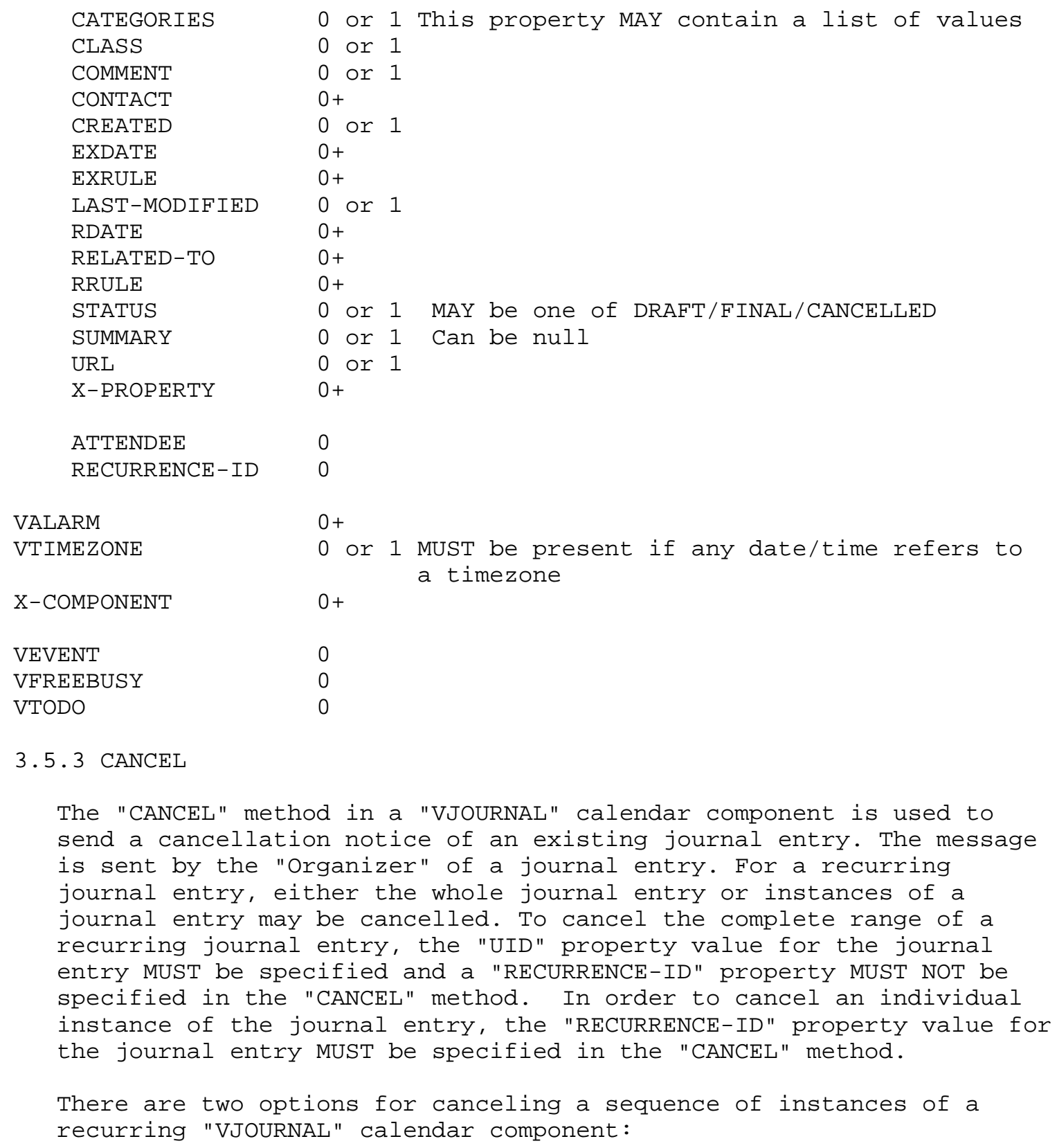


(a) the "RECURRENCE-ID" property for an instance in the sequence MUST be specified with the "RANGE" property parameter value of THISANDPRIOR (or THISANDFUTURE) to indicate cancellation of the specified "VTODO" calendar component and all instances before (or after); or

(b) individual recurrence instances may be cancelled by specifying multiple "RECURRENCE-ID" properties corresponding to the instances to be cancelled.

When a "VJOURNAL" is cancelled, the "SEQUENCE" property value MUST be incremented.

This method type is an icalendar object that conforms to the following property constraints:

\begin{tabular}{|c|c|c|}
\hline Component/Property & Presenc & \\
\hline METHOD & 1 & MUST be "CANCEL" \\
\hline VJOURNAL & $1+$ & All MUST have the same UID \\
\hline DTSTAMP & 1 & \\
\hline ORGANIZER & 1 & \\
\hline SEQUENCE & 1 & \\
\hline UID & 1 & MUST be the UID of the original REQUEST \\
\hline ATTACH & $0+$ & \\
\hline ATTENDEE & $0+$ & \\
\hline CATEGORIES & 0 or 1 & This property MAY contain a list of values \\
\hline CLASS & 0 or 1 & \\
\hline COMMENT & 0 or 1 & \\
\hline CONTACT & $0+$ & \\
\hline CREATED & 0 or 1 & \\
\hline DESCRIPTION & 0 or 1 & \\
\hline DTSTART & 0 or 1 & \\
\hline EXDATE & $0+$ & \\
\hline EXRULE & $0+$ & \\
\hline LAST-MODIFIED & 0 or 1 & \\
\hline $\mathrm{RDATE}$ & $0+$ & \\
\hline RECURRENCE-ID & 0 or 1 & $\begin{array}{l}\text { only if referring to an instance of a } \\
\text { recurring calendar component. Otherwise } \\
\text { it MUST NOT be present. }\end{array}$ \\
\hline RELATED-TO & $0+$ & \\
\hline RRULE & $0+$ & \\
\hline STATUS & 0 or 1 & $\begin{array}{l}\text { MAY be present, must be "CANCELLED" if } \\
\text { present }\end{array}$ \\
\hline SUMMARY & 0 or 1 & \\
\hline URL & 0 or 1 & \\
\hline X-PROPERTY & $0+$ & \\
\hline
\end{tabular}

Silverberg, et. al. Standards Track [Page 54] 
REQUEST-STATUS 0

$\begin{array}{lll}\text { VTIMEZONE } & 0+ & \begin{array}{l}\text { MUST be present if any date/time refers to } \\ \text { a timezone }\end{array} \\ \text { X-COMPONENT } & 0+ & \\ \text { VALARM } & 0 & \\ \text { VEVENT } & 0 & \\ \text { VEREEBUSY } & 0 & \\ \text { VTODO } & 0 & \end{array}$

3.6 Status Replies

The "REQUEST-STATUS" property may include the following values:

\begin{tabular}{|c|c|c|}
\hline $\begin{array}{l}\text { Short Return } \\
\text { Status Code }\end{array}$ & $\begin{array}{l}\text { Longer Return Status } \\
\text { Description }\end{array}$ & Offending Data \\
\hline \multicolumn{3}{|c|}{ 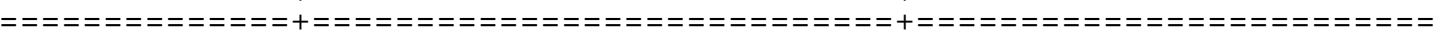 } \\
\hline 2.0 & Success. & \\
\hline \multicolumn{3}{|c|}{ =" } \\
\hline 2.1 & $\begin{array}{l}\text { Success but fallback taken } \\
\text { on one or more property } \\
\text { values. }\end{array}$ & $\begin{array}{l}\text { Property name and value } \\
\text { MAY be specified. }\end{array}$ \\
\hline \multicolumn{3}{|c|}{ 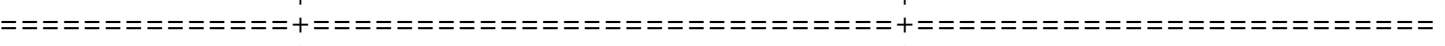 } \\
\hline 2.2 & $\begin{array}{l}\text { Success, invalid property } \\
\text { ignored. }\end{array}$ & $\begin{array}{l}\text { Property name MAY be } \\
\text { specified. }\end{array}$ \\
\hline \multicolumn{3}{|c|}{ 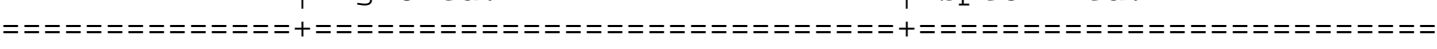 } \\
\hline 2.3 & $\begin{array}{l}\text { Success, invalid property } \\
\text { parameter ignored. }\end{array}$ & $\begin{array}{l}\text { Property parameter name } \\
\text { and value MAY be } \\
\text { specified. }\end{array}$ \\
\hline \multicolumn{3}{|c|}{ 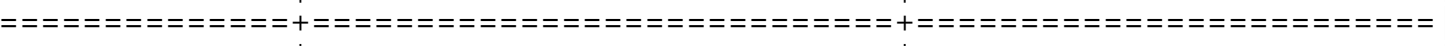 } \\
\hline 2.4 & $\begin{array}{l}\text { Success, unknown non- } \\
\text { standard property ignored. }\end{array}$ & $\begin{array}{l}\text { Non-standard property } \\
\text { name MAY be specified. }\end{array}$ \\
\hline \multicolumn{3}{|c|}{ 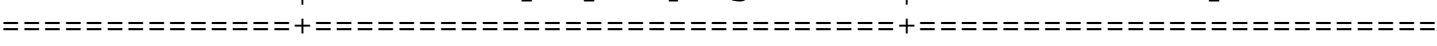 } \\
\hline 2.5 & $\begin{array}{l}\text { Success, unknown non } \\
\text { standard property value } \\
\text { ignored. }\end{array}$ & $\begin{array}{l}\text { Property and non- } \\
\text { standard value MAY be } \\
\text { specified. }\end{array}$ \\
\hline \multicolumn{3}{|c|}{ =" =" =" } \\
\hline 2.6 & $\begin{array}{l}\text { Success, invalid calendar } \\
\text { component ignored. }\end{array}$ & $\begin{array}{l}\text { Calendar component } \\
\text { sentinel (e.g., BEGIN: } \\
\text { ALARM) MAY be } \\
\text { specified. }\end{array}$ \\
\hline \multicolumn{3}{|c|}{ 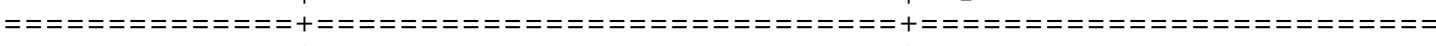 } \\
\hline 2.7 & $\begin{array}{l}\text { Success, request forwarded } \\
\text { to Calendar User. }\end{array}$ & $\begin{array}{l}\text { Original and forwarded } \\
\text { caluser addresses MAY } \\
\text { be specified. }\end{array}$ \\
\hline \multicolumn{3}{|c|}{ 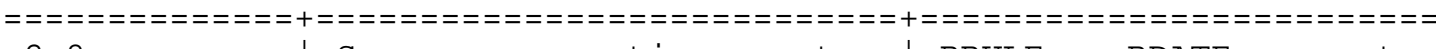 } \\
\hline 2.8 & Success, repeating event & RRULE or RDATE property \\
\hline
\end{tabular}




\begin{tabular}{|c|c|c|}
\hline & $\begin{array}{l}\text { ignored. Scheduled as a } \\
\text { single component. }\end{array}$ & $\begin{array}{l}\text { name and value MAY be } \\
\text { specified. }\end{array}$ \\
\hline \multicolumn{3}{|c|}{ 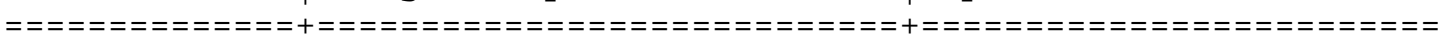 } \\
\hline 2.9 & $\begin{array}{l}\text { Success, truncated end date } \\
\text { time to date boundary. }\end{array}$ & $\begin{array}{l}\text { DTEND property value } \\
\text { MAY be specified. }\end{array}$ \\
\hline \multicolumn{3}{|c|}{$=0$} \\
\hline 2.10 & $\begin{array}{l}\text { Success, repeating VTODO } \\
\text { ignored. Scheduled as a } \\
\text { single VTODO. }\end{array}$ & $\begin{array}{l}\text { RRULE or RDATE property } \\
\text { name and value MAY be } \\
\text { specified. }\end{array}$ \\
\hline \multicolumn{3}{|c|}{ =" =" =" =" } \\
\hline 2.11 & $\begin{array}{l}\text { Success, unbounded RRULE } \\
\text { clipped at some finite } \\
\text { number of instances }\end{array}$ & $\begin{array}{l}\text { RRULE property name and } \\
\text { value MAY be specified. } \\
\text { Number of instances MAY } \\
\text { also be specified. }\end{array}$ \\
\hline \multicolumn{3}{|c|}{ =" =" =" } \\
\hline 3.0 & Invalid property name. & $\begin{array}{l}\text { Property name MAY be } \\
\text { specified. }\end{array}$ \\
\hline \multicolumn{3}{|c|}{ 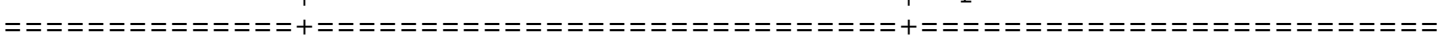 } \\
\hline 3.1 & Invalid property value. & $\begin{array}{l}\text { Property name and value } \\
\text { MAY be specified. }\end{array}$ \\
\hline \multicolumn{3}{|c|}{ 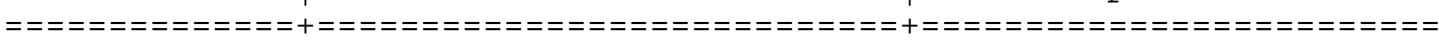 } \\
\hline 3.2 & Invalid property parameter. & $\begin{array}{l}\text { Property parameter name } \\
\text { and value MAY be } \\
\text { specified. }\end{array}$ \\
\hline \multicolumn{3}{|c|}{ =" =" =" } \\
\hline 3.3 & $\begin{array}{l}\text { Invalid property parameter } \\
\text { value. }\end{array}$ & $\begin{array}{l}\text { Property parameter name } \\
\text { and value MAY be } \\
\text { specified. }\end{array}$ \\
\hline \multicolumn{3}{|c|}{ 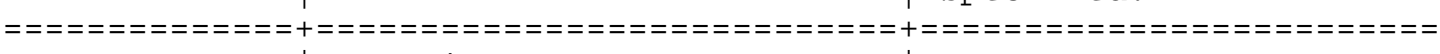 } \\
\hline 3.4 & $\begin{array}{l}\text { Invalid calendar component } \\
\text { sequence. }\end{array}$ & $\begin{array}{l}\text { Calendar component } \\
\text { sentinel MAY be } \\
\text { specified (e.g., BEGIN: } \\
\text { VTIMEZONE). }\end{array}$ \\
\hline \multicolumn{3}{|c|}{ =" =" } \\
\hline 3.5 & Invalid date or time. & $\begin{array}{l}\text { Date/time value (s) MAY } \\
\text { be specified. }\end{array}$ \\
\hline \multicolumn{3}{|c|}{ 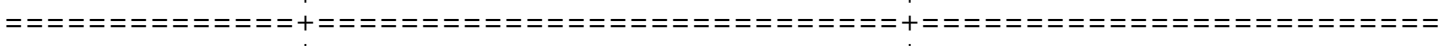 } \\
\hline 3.6 & Invalid rule. & $\begin{array}{l}\text { Rule value MAY be } \\
\text { specified. }\end{array}$ \\
\hline \multicolumn{3}{|c|}{ 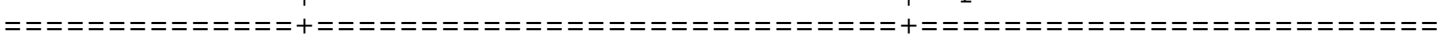 } \\
\hline 3.7 & Invalid Calendar User. & $\begin{array}{l}\text { Attendee property value } \\
\text { MAY be specified. }\end{array}$ \\
\hline \multicolumn{3}{|c|}{ 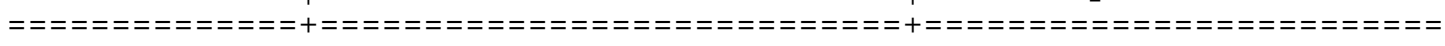 } \\
\hline 3.8 & No authority. & $\begin{array}{l}\text { METHOD and Attendee } \\
\text { property values MAY be } \\
\text { specified. }\end{array}$ \\
\hline
\end{tabular}




\begin{tabular}{|c|c|c|}
\hline 3.9 & Unsupported version. & $\begin{array}{l}\text { VERSION property name } \\
\text { and value MAY be } \\
\text { specified. }\end{array}$ \\
\hline \multicolumn{3}{|c|}{ 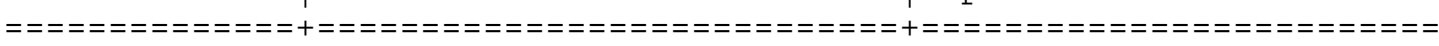 } \\
\hline 3.10 & Request entity too large. & None. $\quad$ N \\
\hline \multicolumn{3}{|c|}{ 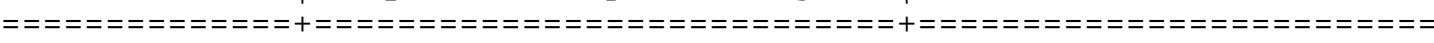 } \\
\hline 3.11 & $\begin{array}{l}\text { Required component or } \\
\text { property missing. }\end{array}$ & $\begin{array}{l}\text { Component or property } \\
\text { name MAY be specified. }\end{array}$ \\
\hline \multicolumn{3}{|c|}{ 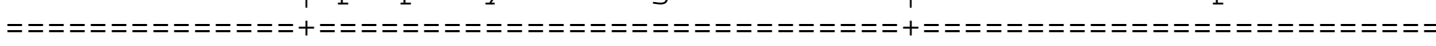 } \\
\hline 3.12 & $\begin{array}{l}\text { Unknown component or } \\
\text { property found }\end{array}$ & $\begin{array}{l}\text { Component or property } \\
\text { name MAY be specified }\end{array}$ \\
\hline \multicolumn{3}{|c|}{ 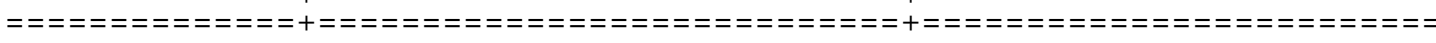 } \\
\hline 3.13 & $\begin{array}{l}\text { Unsupported component or } \\
\text { property found }\end{array}$ & $\begin{array}{l}\text { Component or property } \\
\text { name MAY be specified }\end{array}$ \\
\hline \multicolumn{3}{|c|}{ =" =" } \\
\hline 3.14 & Unsupported capability & $\begin{array}{l}\text { Method or action MAY } \\
\text { be specified }\end{array}$ \\
\hline \multicolumn{3}{|c|}{ 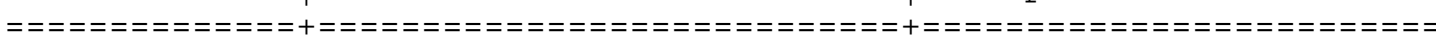 } \\
\hline 4.0 & $\begin{array}{l}\text { Event conflict. Date/time } \\
\text { is busy. }\end{array}$ & $\begin{array}{l}\text { DTSTART and DTEND } \\
\text { property name and values } \\
\text { MAY be specified. }\end{array}$ \\
\hline \multicolumn{3}{|c|}{ 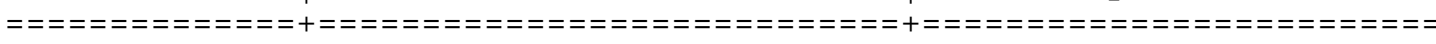 } \\
\hline 5.0 & Request MAY supported. & $\begin{array}{l}\text { Method property value } \\
\text { MAY be specified. }\end{array}$ \\
\hline \multicolumn{3}{|c|}{ =" =" =" } \\
\hline 5.1 & Service unavailable. & $\begin{array}{l}\text { ATTENDEE property value } \\
\text { MAY be specified. }\end{array}$ \\
\hline \multicolumn{3}{|c|}{ 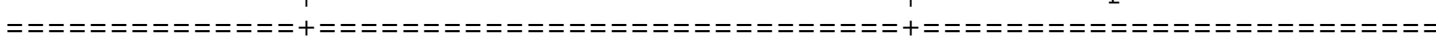 } \\
\hline 5.2 & Invalid calendar service. & $\begin{array}{l}\text { ATTENDEE property value } \\
\text { MAY be specified. }\end{array}$ \\
\hline \multicolumn{3}{|c|}{ 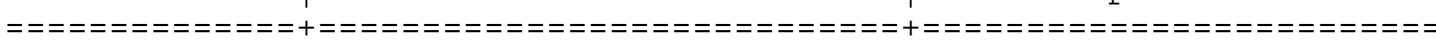 } \\
\hline 5.3 & $\begin{array}{l}\text { No scheduling support for } \\
\text { user. }\end{array}$ & $\begin{array}{l}\text { ATTENDEE property value } \\
\text { MAY be specified. }\end{array}$ \\
\hline
\end{tabular}

\subsection{Implementation Considerations}

\subsubsection{Working With Recurrence Instances}

iCalendar includes a recurrence grammar to represent recurring events. The benefit of such a grammar is the ability to represent a number of events in a single object. However, while this simplifies creation of a recurring event, meeting instances still need to be referenced. For instance, an "Attendee" may decline the third instance of a recurring Friday event. Similarly, the "Organizer" may change the time or location to a single instance of the recurring event. 
Since implementations may elect to store recurring events as either a single event object or a collection of discreet, related event objects, the protocol is designed so that each recurring instance may be both referenced and versioned. Hence, implementations that choose to maintain per-instance properties (such as "ATTENDEE" property "partstat" parameter) may do so. However, the protocol does not require per-instance recognition unless the instance itself must be renegotiated.

The scenarios for recurrence instance referencing are listed below. For purposes of simplification a change to an event refers to a "trigger property." That is, a property that has a substantive effect on the meeting itself such as start time, location, due date (for "VTODO" calendar component components) and possibly description.

"Organizer" initiated actions:

- "Organizer" deletes or changes a single instance of a recurring event

- "Organizer" makes changes that affect all future instances

- "Organizer" makes changes that affect all previous instances

- "Organizer" deletes or modifies a previously changed instance

"Attendee" initiated actions:

- "Attendee" changes status for a particular recurrence instance

- "Attendee" sends Event-Counter for a particular recurrence instance

An instance of a recurring event is assigned a unique identification, "RECURRENCE-ID" property, when that instance is renegotiated. Negotiation may be necessary when a substantive change to the event or to-do has be made (such as changing the start time, end time, due date or location). The "Organizer" can identify a specific recurrence instance using the "RECURRENCE-ID" property. The property value is equal to the date/time of the instance. If the "Organizer" wishes to change the "DTSTART", the original "DTSTART" value is used for "RECURRENCE-ID" property and the new "DTSTART" and "DTEND" values reflect the change. Note that after the change has occurred, the "RECURRENCE-ID" has changed to the new "DTSTART" value.

\subsubsection{Attendee Property Considerations}

The "ORGANIZER" property is required on published events, to-dos, and journal entries for two reasons. First, only the "Organizer" is allowed to update and redistribute an event or to-do component. It follows that the "ORGANIZER" property MUST be present in the event, to-do, or journal entry component so that the CUA has a basis for 
authorizing an update. Second, it is prudent to provide a point of contact for anyone who receives a published component in case of problems.

There are valid [RFC-822] addresses that represent groups. Sending email to such an address results in mail being sent to multiple recipients. Such an address may be used as the value of an "ATTENDEE" property. Thus, it is possible that the recipient of a "REQUEST" does not appear explicitly in the list.

It is recommended that the general approach to finding a "Calendar User" in an attendee list be as follows:

1. Search for the "Calendar User" in the attendee list where "TYPE=INDIVIDUAL"

2. Failing (1) look for attendees where "TYPE=GROUP" or 'TYPE=UNKNOWN". The CUA then determines if the "Calendar User" is a member of one of these groups. If so, the "REPLY" method sent to the "Organizer" MUST contain a new "ATTENDEE" property in which:

- the "type" property parameter is set to INDIVIDUAL

- the "member" property parameter is set to the name of the group

3. Failing (2) the CUA MAY ignore or accept the request as the "Calendar User" wishes.

\section{$3.7 .3 \mathrm{x}$-Tokens}

To make icalendar objects extensible, new property types MAY be inserted into components. These properties are called X-Tokens as they are prefixed with "X-". A client is not required to make sense of $\mathrm{X}$-Tokens. Clients are not required to save $\mathrm{X}$-Tokens or use them in replies.

4 Examples

\subsection{Published Event Examples}

In the calendaring and scheduling context, publication refers to the one way transfer of event information. Consumers of published events simply incorporate the event into a calendar. No reply is expected. Individual "A" publishes an event. Individual "B" reads the event and incorporates it into their calendar. Events are published in several ways including: embedding the event as an object in a web page, emailing the event to a distribution list, and posting the event to a newsgroup. 
The table below illustrates the sequence of events between the publisher and the consumers of a published event.

\begin{tabular}{|c|c|}
\hline Action & "Organizer" \\
\hline Publish an event & $\begin{array}{l}\text { "A" sends or posts a PUBLISH } \\
\text { message }\end{array}$ \\
\hline "B" reads a published event & \\
\hline Publish an updated event & $\begin{array}{l}\text { "A" sends or posts a PUBLISH } \\
\text { message }\end{array}$ \\
\hline "B" reads the updated event & \\
\hline Cancel a published event & $\begin{array}{l}\text { "A" sends or posts a CANCEL } \\
\text { message }\end{array}$ \\
\hline $\begin{array}{l}\text { "B" reads the canceled event } \\
\text { publication }\end{array}$ & \\
\hline
\end{tabular}

4.1.1 A Minimal Published Event

The icalendar object below describes a single event that begins on July 1, 1997 at 20:00 UTC. This event contains the minimum set of properties for a "PUBLISH" for a "VEVENT" calendar component.

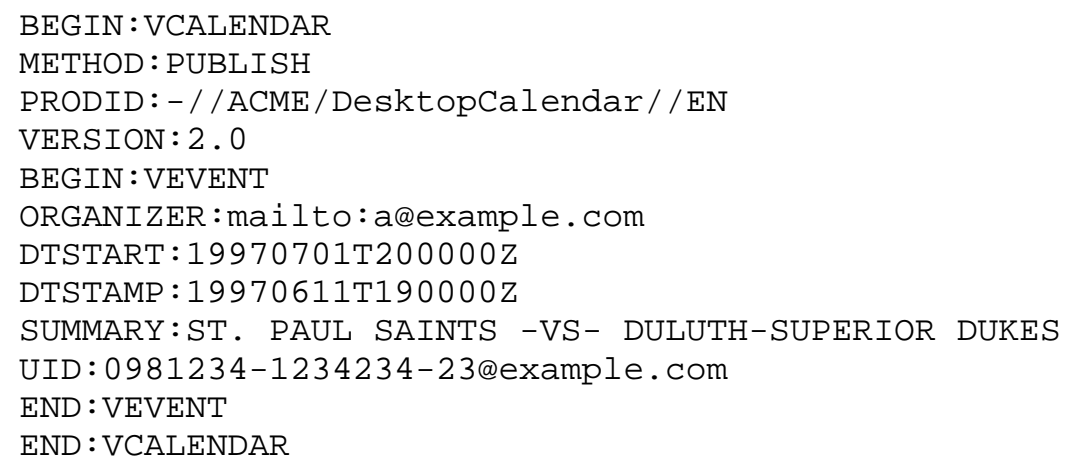

\subsubsection{Changing A Published Event}

The icalendar object below describes an update to the event described in 4.1.1, the time has been changed, an end time has been added, and the sequence number has been adjusted. 


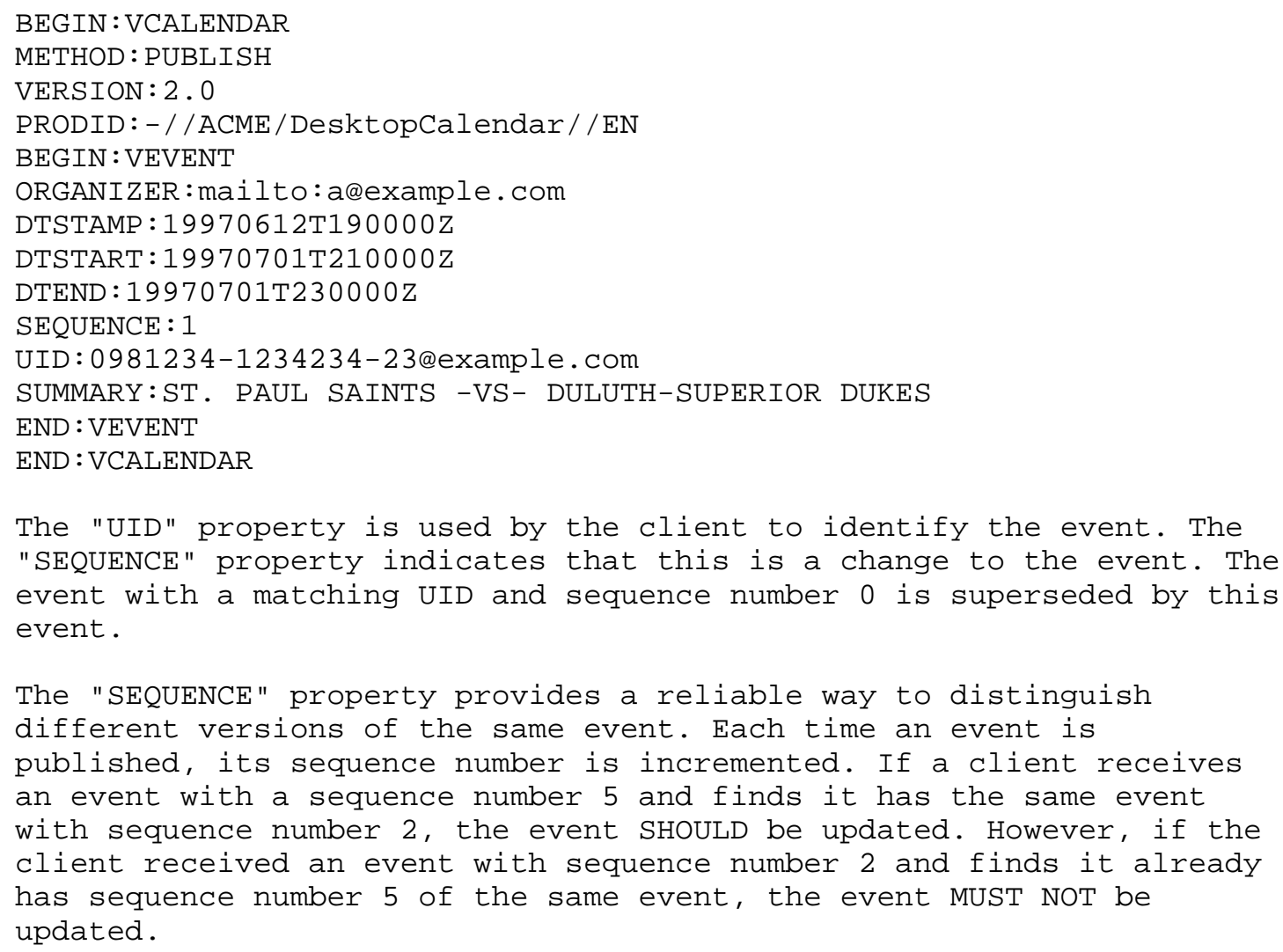

4.1.3 Canceling A Published Event

The icalendar object below cancels the event described in 4.1.1. This cancels the event with "SEQUENCE" property of 0, 1, and 2 .

BEGIN : VCALENDAR

METHOD : CANCEL

VERSION : 2.0

PRODID :-//ACME/DesktopCalendar//EN

BEGIN : VEVENT

ORGANIZER:mailto: a example.com

COMMENT:DUKES forfeit the game

SEQUENCE : 2

UID : 0981234-1234234-23@example.com

DTSTAMP : $19970613 \mathrm{~T} 190000 \mathrm{Z}$

END : VEVENT

END : VCALENDAR 
4.1.4 A Rich Published Event

This example describes the same event as in 4.1.1, but in much greater detail.

BEGIN : VCALENDAR

PRODID : - / /ACME/DesktopCalendar//EN

METHOD : PUBLISH

SCALE : GREGORIAN

VERSION : 2 . 0

BEGIN : VTIMEZONE

TZID:America-Chicago

TZURL: http://zones.stds_r_us.net/tz/America-Chicago

BEGIN : STANDARD

DTSTART : $19671029 T 020000$

RRULE : $F R E Q=Y E A R L Y ; B Y D A Y=-1 S U ; B Y M O N T H=10$

TZOFFSETFROM:-0500

TZOFFSETTO:-0600

TZNAME : CST

END : STANDARD

BEGIN : DAYLIGHT

DTSTART : $19870405 T 020000$

RRULE : $F R E Q=Y E A R L Y ; B Y D A Y=1 S U$; $B Y M O N T H=4$

TZOFFSETFROM: -0600

TZOFFSETTO: -0500

TZNAME : CDT

END : DAYLIGHT

END : VTIMEZONE

BEGIN : VEVENT

ORGANIZER:mailto:a@example.com

ATTACH: http://www. dukes.com/

CATEGORIES:SPORTS EVENT, ENTERTAINMENT

CLASS :PRIVATE

DESCRIPTION:MIDWAY STADIUM $\backslash n$

Big time game. MUST see. $\backslash \mathrm{n}$

Expected duration:2 hours $\backslash \mathrm{n}$

DTEND; TZID=America-Chicago:19970701T180000

DTSTART; TZID=America-Chicago: $19970702 \mathrm{~T} 160000$

DTSTAMP : $19970614 \mathrm{~T} 190000 \mathrm{Z}$

STATUS : CONF IRMED

LOCATION; VALUE=URI : http: / / www . midwaystadium.com/

PRIORITY : 2

RESOURCES : SCOREBOARD

SEQUENCE : 3

SUMMARY:ST. PAUL SAINTS -VS- DULUTH-SUPERIOR DUKES

UID : 0981234-1234234-23 eexample.com

RELATED-TO:0981234-1234234-14@example.com

BEGIN : VALARM 
TRIGGER: $-\mathrm{PT} 2 \mathrm{H}$

ACTION : DISPLAY

DESCRIPTION:You should be leaving for the game now.

END : VALARM

BEGIN : VALARM

TRIGGER:-PT30M

ACTION : AUDIO

END : VALARM

END : VEVENT

END : VCALENDAR

The "RELATED-TO" field contains the "UID" property of a related calendar event. The "SEQUENCE" property 3 indicates that this event supersedes versions 0,1 , and 2 .

4.1.5 Anniversaries or Events attached to entire days

This example demonstrates the use of the "value" parameter to tie a "VEVENT" to day rather than a specific time.

BEGIN: VCALENDAR

PRODID :-//ACME/DesktopCalendar//EN

METHOD : PUBLISH

VERSION:2.0

BEGIN: VEVENT

ORGANIZER:mailto:a@example.com

DTSTAMP $: 19970614 \mathrm{~T} 190000 \mathrm{Z}$

UID:0981234-1234234-23@example.com

DTSTART; VALUE $=$ DATE $: 19970714$

RRULE : $F R E Q=Y E A R L Y ;$ INTERVAL=1

SUMMARY: Bastille Day

END : VEVENT

END : VCALENDAR

4.2 Group Event Examples

Group events are distinguished from published events in that they have "Attendees" and that there is interaction between the

"Attendees" and the "Organizer" with respect to the event. Individual "A" requests a meeting between individuals "A", "B", "C" and "D". Individual "B" confirms attendance to the meeting. Individual "C" declines attendance. Individual "D" tentatively confirms attendance. The following table illustrates the the message flow between these individuals. A, the CU scheduling the meeting, is referenced as the "Organizer". 


\begin{tabular}{|c|c|c|}
\hline Action & "Organizer" & Attendee \\
\hline $\begin{array}{l}\text { Initiate a meeting } \\
\text { request }\end{array}$ & $\begin{array}{l}\text { "A" sends a REQUEST } \\
\text { message to "B", "C", } \\
\text { and "D" }\end{array}$ & \\
\hline $\begin{array}{l}\text { Accept the meeting } \\
\text { request }\end{array}$ & & $\begin{array}{l}\text { "B" sends a REPLY } \\
\text { message to "A" with its } \\
\text { ATTENDEE "partstat" para- } \\
\text { set to "accepted" }\end{array}$ \\
\hline $\begin{array}{l}\text { Decline the meeting } \\
\text { request }\end{array}$ & & $\begin{array}{l}\text { "C" sends a REPLY } \\
\text { message to "A" with its } \\
\text { ATTENDEE "partstat" para- } \\
\text { set to "declined" }\end{array}$ \\
\hline $\begin{array}{l}\text { Tentatively accept } \\
\text { the meeting request }\end{array}$ & & $\begin{array}{l}\text { "D" sends a REPLY } \\
\text { message to "A" with its } \\
\text { ATTENDEE "partstat" para- } \\
\text { set to "tentative" }\end{array}$ \\
\hline $\begin{array}{l}\text { Confirm meeting } \\
\text { status with } \\
\text { attendees }\end{array}$ & $\begin{array}{l}\text { "A" sends a REQUEST } \\
\text { message to "B" and } \\
\text { "D" with updated } \\
\text { information. }\end{array}$ & \\
\hline
\end{tabular}

\subsubsection{A Group Event Request}

A sample meeting request is sent from "A" to "B", "C", and "D". _E_ is also sent a copy of the request but is not expected to attend and need not reply. "E" illustrates how CUAs might implement an "FYI" type feature. Note the use of the "role" parameter. The default value for the "role" parameter is "req-participant" and it need not be enumerated. In this case we are using the value "non-participant" to indicate "E" is a non-attending $\mathrm{CU}$. The parameter is not needed on other "Attendees" since "participant" is the default value.

BEGIN : VCALENDAR

PRODID : - / / ACME/DesktopCalendar//EN

METHOD : REQUEST

VERSION : 2 . 0

BEGIN : VEVENT

ORGANIZER:Mailto:A@example.com

ATTENDEE; ROLE=CHAIR;PARTSTAT=ACCEPTED;CN=BIG A:Mailto:A@example.com ATTENDEE; RSVP=TRUE; TYPE=INDIVIDUAL; CN=B :Mailto : Bdexample.com ATTENDEE; RSVP=TRUE; TYPE=INDIVIDUAL; CN=C :Mailto : C@example. com 
ATTENDEE; RSVP=TRUE; TYPE=INDIVIDUAL; CN=Hal : Mailto:D@example.com

ATTENDEE; RSVP=FALSE; TYPE=ROOM : conf_Big@example.com

ATTENDEE; ROLE=NON-PARTICIPANT; RSVP=FALSE : Mailto : E@example.com DTSTAMP : $19970611 \mathrm{~T} 190000 \mathrm{Z}$

DTSTART : $19970701 \mathrm{~T} 200000 \mathrm{Z}$

DTEND: $19970701 \mathrm{~T} 2000000 \mathrm{Z}$

SUMMARY: Conference

UID:calsrv.example.com-873970198738777@example.com

SEQUENCE : 0

STATUS : CONF IRMED

END : VEVENT

END : VCALENDAR

4.2.2 Reply To A Group Event Request

Attendee "B" accepts the meeting.

BEGIN : VCALENDAR

PRODID :-//ACME/DesktopCalendar//EN

METHOD : REPLY

VERSION : 2 . 0

BEGIN : VEVENT

ATTENDEE; PARTSTAT=ACCEPTED:Mailto:B@example.com

ORGANIZER : MAILTO:A@example.com

UID:calsrv.example.com-873970198738777@example.com

SEQUENCE : 0

REQUEST-STATUS: 2 .0; Success

DTSTAMP : $19970612 \mathrm{~T} 190000 \mathrm{Z}$

END : VEVENT

END : VCALENDAR

"B" could have declined the meeting or indicated tentative acceptance by setting the "ATTENDEE" "partstat" parameter to "declined" or "tentative", respectively. Also, "REQUEST-STATUS" is not required in successful transactions.

4.2.3 Update An Event

The event is moved to a different time. The combination of the "UID" property (unchanged) and the "SEQUENCE" (bumped to 1) properties indicate the update.

BEGIN : VCALENDAR

PRODID :-//ACME/DesktopCalendar//EN

METHOD : REQUEST

VERSION : 2 . 0

BEGIN : VEVENT

ORGANIZER:Mailto:A@example.com

Silverberg, et. al

Standards Track

[Page 65] 
ATTENDEE; ROLE=CHAIR;PARTSTAT=ACCEPTED : Mailto: A@example. com

ATTENDEE; RSVP=TRUE; TYPE=INDIVIDUAL : Mailto:B@example.com

ATTENDEE; RSVP=TRUE; TYPE=INDIVIDUAL : Mail to: C@example.com

ATTENDEE; RSVP=TRUE; TYPE=INDIVIDUAL; CN=Hal : Mailto:D@example.com

ATTENDEE; ROLE=NON-PARTICIPANT; RSVP=FALSE;

CUTYPE=ROOM:Mailto: Conf@example.com

ATTENDEE; ROLE=NON-PARTICIPANT; RSVP=FALSE : Mailto: edexample. com

DTSTART : $19970701 \mathrm{~T} 180000 \mathrm{Z}$

DTEND : $19970701 \mathrm{~T} 190000 \mathrm{Z}$

SUMMARY:Phone Conference

UID:calsrv.example.com-873970198738777@example.com

SEQUENCE : 1

DTSTAMP : $19970613 \mathrm{~T} 190000 \mathrm{Z}$

STATUS : CONF IRMED

END : VEVENT

END : VCALENDAR

4.2.4 Countering an Event Proposal

"A" sends a "REQUEST" to "B" and "C". "B" makes a counter-proposal to "A" to change the time and location.

"A" sends the following "REQUEST":

BEGIN : VCALENDAR

PRODID :-//ACME/DesktopCalendar//EN

METHOD : REQUEST

VERSION : 2 . 0

BEGIN : VEVENT

ORGANIZER:Mailto:A@example.com

ATTENDEE; ROLE=CHAIR; PARTSTAT=ACCEPTED : Mailto:A@example.com

ATTENDEE; RSVP=TRUE; TYPE=INDIVIDUAL : Mail to: B@example.com

ATTENDEE; RSVP=TRUE; TYPE=INDIVIDUAL : Mail to: C@example.com DTSTART : $19970701 \mathrm{~T} 190000 \mathrm{Z}$

DTEND : $19970701 \mathrm{~T} 200000 \mathrm{Z}$

SUMMARY:Discuss the Merits of the election results

LOCATION:Green Conference Room

UID : calsrv.example.com-873970198738777a@example.com

SEQUENCE : 0

DTSTAMP : $19970611 \mathrm{~T} 190000 \mathrm{Z}$

STATUS : CONF IRMED

END : VEVENT

END : VCALENDAR

"B" sends "COUNTER" to "A", requesting changes to time and place. "B" uses the "COMMENT" property to communicate a rationale for the change. Note that the "SEQUENCE" property is NOT incremented on a "COUNTER". 


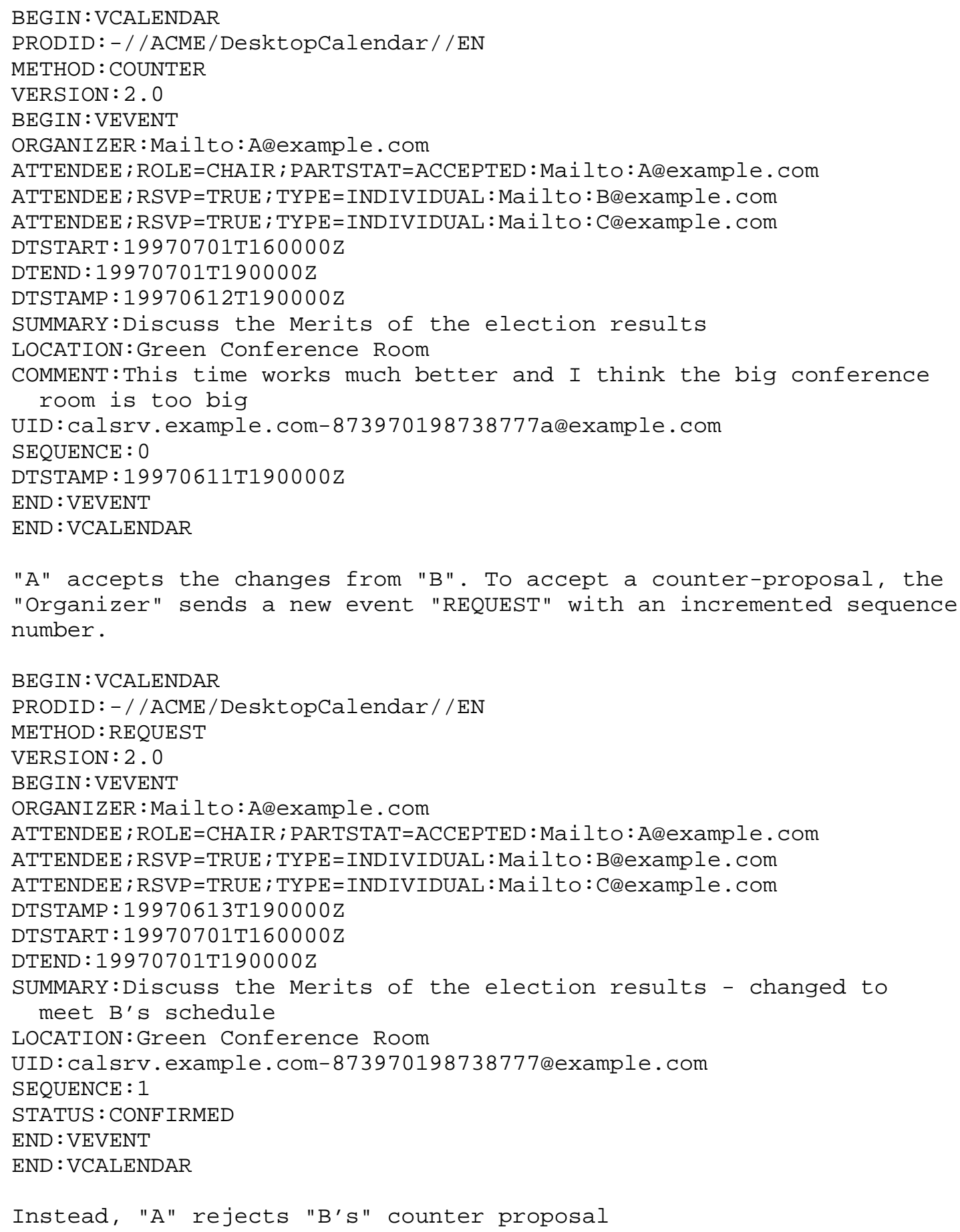




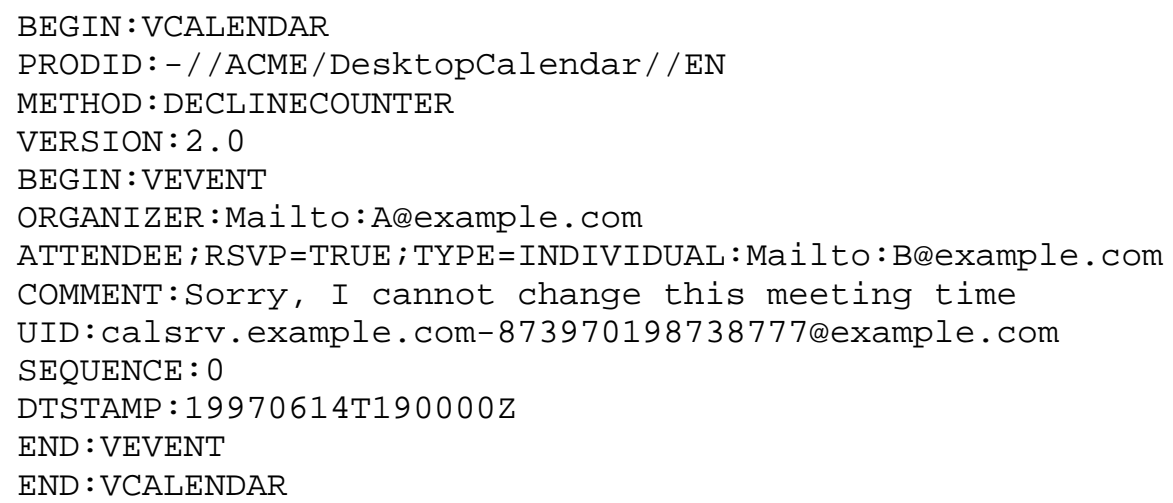

\subsubsection{Delegating an Event}

When delegating an event request to another "Calendar User", the "Delegator" must both update the "Organizer" with a "REPLY" and send a request to the "Delegate". There is currently no protocol limitation to delegation depth. It is possible for the original

delegate to delegate the meeting to someone else, and so on. When a request is delegated from one CUA to another there are a number of responsibilities required of the "Delegator". The "Delegator" MUST:

- Send a "REPLY" to the "Organizer" with the following updates:

- The "Delegator's" "ATTENDEE" property "partstat" parameter set to "delegated" and the "delegated-to" parameter is set to the address of the "Delegate"

- Add an additional "ATTENDEE" property for the "Delegate" with the "delegated-from" property parameter set to the "Delegator"

- Indicate whether they want to continue to receive updates when the "Organizer" sends out updated versions of the event. Setting the "rsvp" property parameter to "TRUE" will cause the updates to be sent, setting it to "FALSE" causes no further updates to be sent. Note that in either case, if the "Delegate" declines the invitation the "Delegator" will be notified.

- The "Delegator" MUST also send a copy of the original "REQUEST" method to the "Delegate".

It is not required that the "Delegate" include the "Delegator" in their "REPLY" method. However, it is strongly advised since this will inform the "Delegator" whether the "Delegate" plans to attend the meeting. [Editors note: How so?] If the "Delegate" declines the meeting, the "Delegator" may elect to delegate the "REQUEST" to another CUA. The process is the same. 


\begin{tabular}{|c|c|c|}
\hline Action & "Organizer" & Attendee \\
\hline $\begin{array}{l}\text { Initiate a meeting } \\
\text { request }\end{array}$ & $\begin{array}{l}\text { "A" sends a REQUEST } \\
\text { message to "B" and } \\
\text { "C" }\end{array}$ & \\
\hline $\begin{array}{l}\text { Delegate: } \\
\text { "C" delegates to } \\
\text { "E" }\end{array}$ & & $\begin{array}{l}\text { "C" sends a REPLY to "A" } \\
\text { with the ATTENDEE. } \\
\text { "partstat" parameter set } \\
\text { to "delegated" and with a } \\
\text { new "ATTENDEE" property } \\
\text { for "E". "E's" ATTENDEE } \\
\text { "delegated-from" param } \\
\text { is set to "C". "C's" } \\
\text { ATTENDEE "delegated-to" } \\
\text { param is set to "E". } \\
\text { "C" sends REQUEST message } \\
\text { to "E" with the original } \\
\text { meeting request } \\
\text { information. The } \\
\text { "partstat" property } \\
\text { parameter for "C" is set } \\
\text { to "delegated" and the } \\
\text { "delegated-to" } \\
\text { parameter is set to } \\
\text { the address of "E". An } \\
\text { "ATtENDEE" property is } \\
\text { added for "E" and the } \\
\text { "delegated-from" } \\
\text { parameter is set to } \\
\text { the address of "C". }\end{array}$ \\
\hline $\begin{array}{l}\text { Confirm meeting } \\
\text { attendance }\end{array}$ & & $\begin{array}{l}\text { "E" sends REPLY message } \\
\text { to "A" and optionally "C" } \\
\text { with its "partstat" } \\
\text { property parameter set } \\
\text { to "ACCEPTED" }\end{array}$ \\
\hline $\begin{array}{l}\text { Optional: } \\
\text { Redistribute } \\
\text { meeting to } \\
\text { attendees }\end{array}$ & $\begin{array}{l}\text { "A" sends REQUEST } \\
\text { message to "B", "C" } \\
\text { and "E". }\end{array}$ & \\
\hline
\end{tabular}




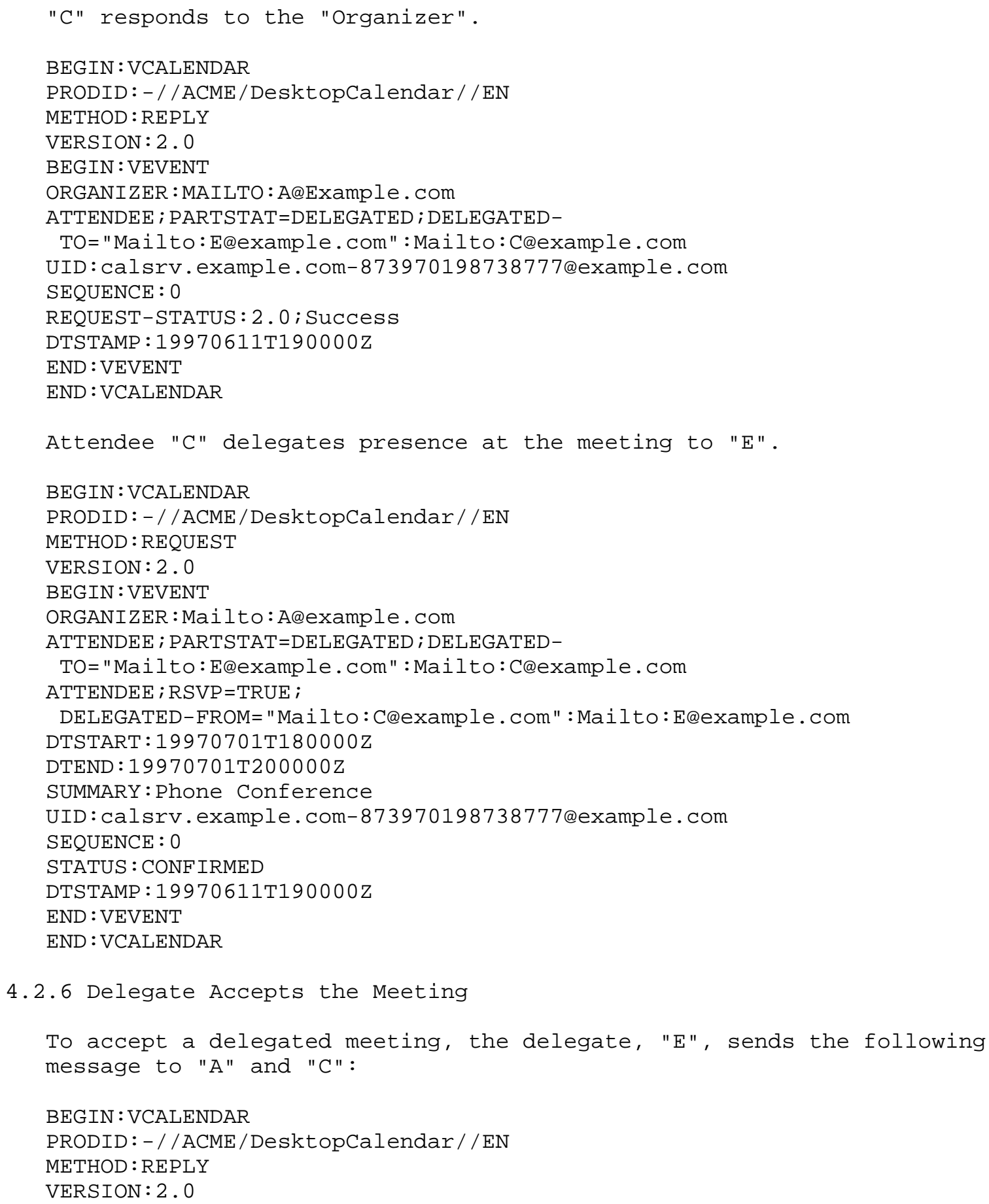




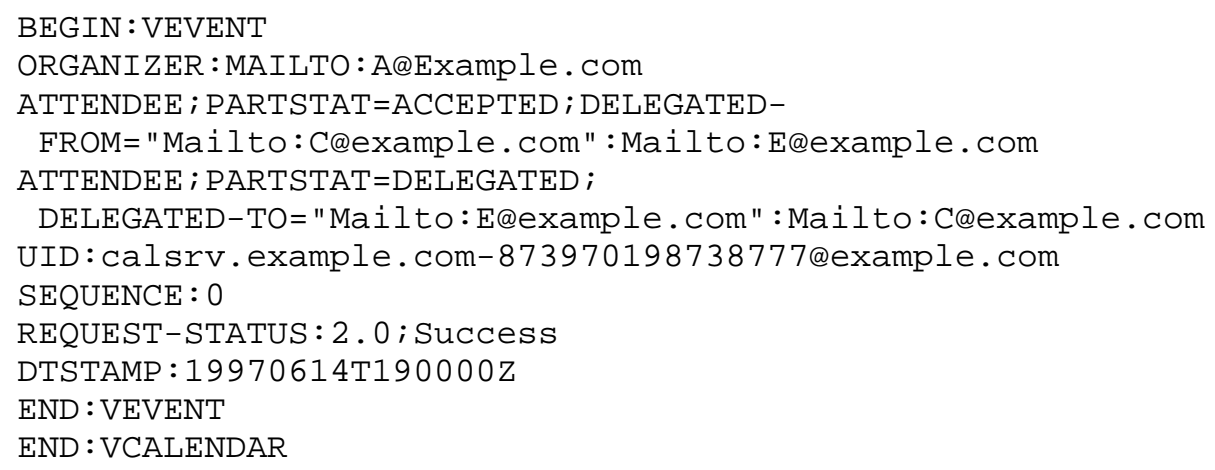




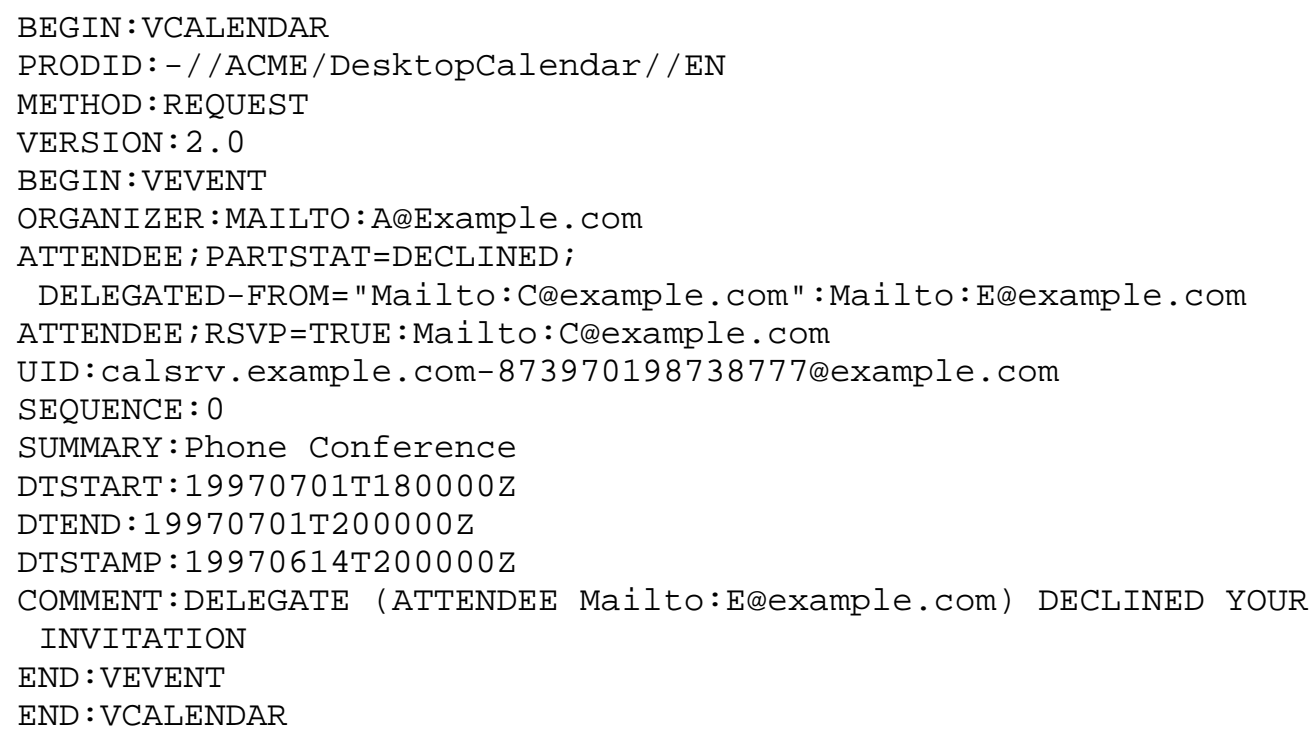

\subsubsection{Forwarding an Event Request}

The protocol does not prevent an "Attendee" from "forwarding" an "VEVENT" calendar component to other "Calendar Users". Forwarding differs from delegation in that the forwarded "Calendar User" (often referred to as a "Party Crasher") does not replace the forwarding "Calendar User". Implementations are not required to add the "Party Crasher" to the "Attendee" list and hence there is no guarantee that a "Party Crasher" will receive additional updates to the Event. The forwarding "Calendar User" SHOULD NOT add the "Party Crasher" to the attendee list. The "Organizer" MAY add the forwarded "Calendar User" to the attendee list.

\subsubsection{Cancel A Group Event}

Individual "A" requests a meeting between individuals "A", "B", "C", and "D". Individual "B" declines attendance to the meeting. Individual "A" decides to cancel the meeting. The following table illustrates the sequence of messages that would be exchanged between these individuals.

Messages related to a previously canceled event ("SEQUENCE" property value is less than the "SEQUENCE" property value of the "CANCEL" message) MUST be ignored. 


\begin{tabular}{|c|c|c|}
\hline Action & "Organizer" & "Attendee" \\
\hline $\begin{array}{l}\text { Initiate a meeting } \\
\text { request }\end{array}$ & $\begin{array}{l}\text { "A" sends a REQUEST } \\
\text { message to "B", "C", } \\
\text { and "D" }\end{array}$ & \\
\hline $\begin{array}{l}\text { Decline the meeting } \\
\text { request }\end{array}$ & & $\begin{array}{l}\text { "B" sends a "REPLY" } \\
\text { message to "A" with its } \\
\text { "partstat" para- } \\
\text { set to "declined". }\end{array}$ \\
\hline Cancel the meeting & $\begin{array}{l}\text { "A" sends a CANCEL } \\
\text { message to "B", "C" } \\
\text { and "D" }\end{array}$ & \\
\hline
\end{tabular}

The example shows how "A" cancels the event.

BEGIN : VCALENDAR

PRODID : - / / ACME/DesktopCalendar//EN

METHOD : CANCEL

VERSION : 2 . 0

BEGIN : VEVENT

ORGANIZER:Mailto:A@example.com

ATTENDEE; TYPE=INDIVIDUAL; Mailto:A@example.com

ATTENDEE; TYPE=INDIVIDUAL:Mailto:B@example.com

ATTENDEE; TYPE=INDIVIDUAL:Mailto: C@example.com

ATTENDEE; TYPE=INDIVIDUAL:Mailto:D@example.com

COMMENT:Mr. B cannot attend. It's raining. Lets cancel.

UID:calsrv. example.com-873970198738777@example.com

SEQUENCE : 1

STATUS : CANCELLED

DTSTAMP : $19970613 \mathrm{~T} 190000 \mathrm{Z}$

END : VEVENT

END : VCALENDAR 


\subsubsection{Removing Attendees}

"A" wants to remove "B" from a meeting. This is done by sending a "CANCEL" to "B" and removing "B" from the attendee list in the master copy of the event.

\begin{tabular}{|c|c|c|}
\hline Action & "Organizer" & "Attendee" \\
\hline $\begin{array}{l}\text { Remove an "B" } \\
\text { as an "Attendee" }\end{array}$ & $\begin{array}{l}\text { "A" sends a CANCEL } \\
\text { message to "B" }\end{array}$ & \\
\hline $\begin{array}{l}\text { Update the master } \\
\text { copy of the event }\end{array}$ & $\begin{array}{l}\text { "A" sends the } \\
\text { updated event to } \\
\text { the remaining } \\
\text { "Attendees" }\end{array}$ & \\
\hline
\end{tabular}

The original meeting includes "A", "B", "C", and "D". The example below shows the "CANCEL" that "A" sends to "B". Note that in the example below the "STATUS" property is omitted. This is used when the meeting itself is cancelled and not when the intent is to remove an "Attendee" from the Event.

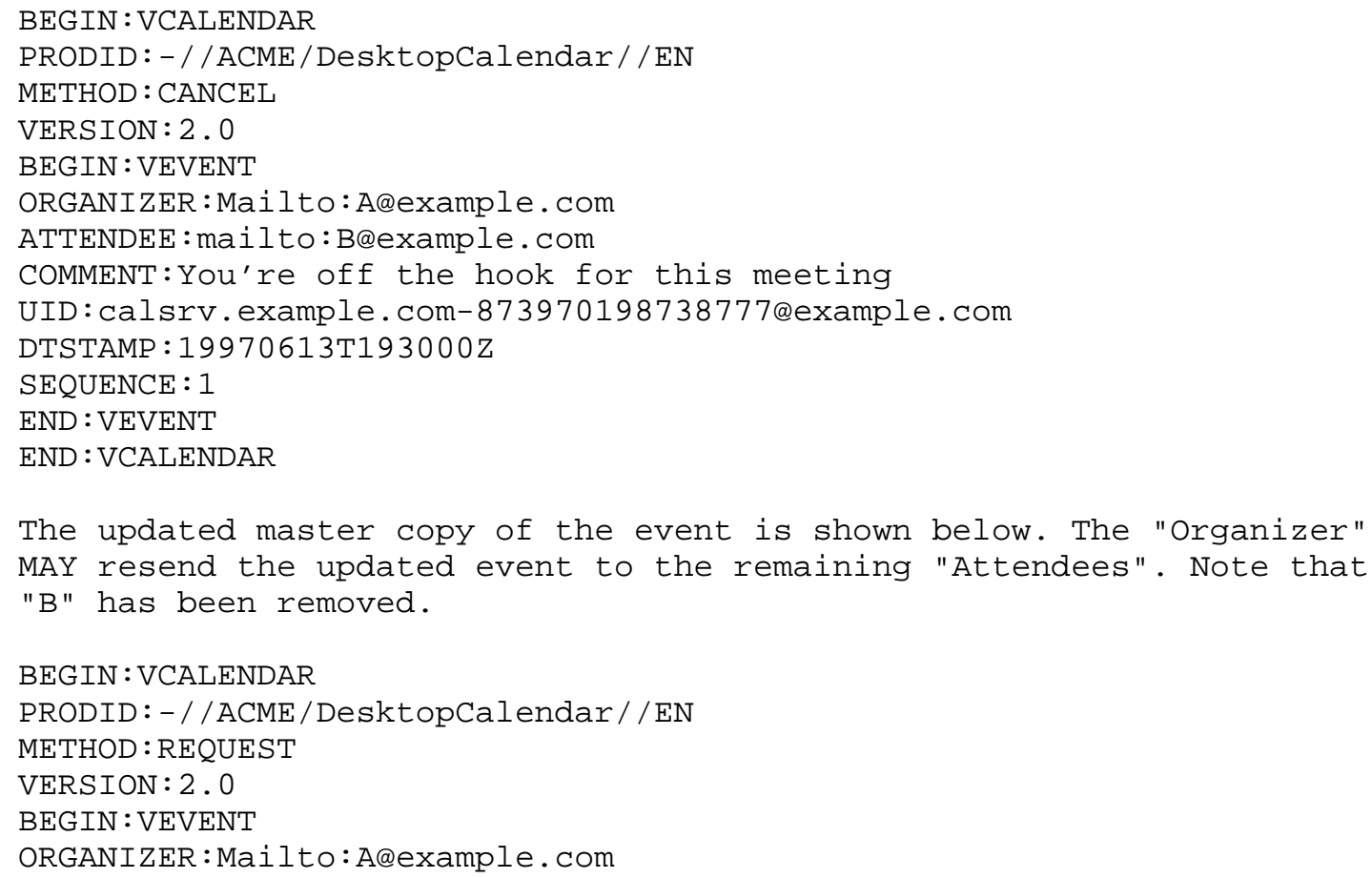


ATTENDEE; ROLE=CHAIR;PARTSTAT=ACCEPTED : Mailto:A@example $\cdot$ com

ATTENDEE; TYPE=INDIVIDUAL:Mailto: CQexample.com

ATTENDEE; TYPE=INDIVIDUAL:Mailto:D@example.com

ATTENDEE; TYPE=ROOM:CR_Bigdexample.com

ATTENDEE; ROLE $=$ NON-PARTICIPANT;

RSVP=FALSE:Mailto: E@example.com

DTSTAMP : $19970611 \mathrm{~T} 190000 \mathrm{Z}$

DTSTART: $19970701 \mathrm{~T} 200000 \mathrm{Z}$

DTEND : $19970701 \mathrm{~T} 203000 \mathrm{Z}$

SUMMARY:Phone Conference

UID:calsrv.example.com-873970198738777@example.com

SEQUENCE : 2

STATUS : CONF IRMED

END : VEVENT

END : VCALENDAR

4.2.11 Replacing the Organizer

The scenario for this example begins with "A" as the "Organizer" for a recurring meeting with "B", "C", and "D". "A" receives a new job offer in another country and drops out of touch. "A" left no forwarding address or way to be reached. Using out-of-band communication, the other "Attendees" eventually learn what has happened and reach an agreement that "B" should become the new "Organizer" for the meeting. To do this, "B" sends out a new version of the event and the other "Attendees" agree to accept "B" as the new "Organizer". "B" also removes "A" from the event.

When the "Organizer" is replaced, the "SEQUENCE" property value MUST be incremented.

This is the message "B" sends to "C" and "D"

BEGIN : VCALENDAR

PRODID :- / / ACME/DesktopCalendar//EN

METHOD : REQUEST

VERSION : 2 . 0

BEGIN : VEVENT

ORGANIZER:Mailto:B@example.com

ATTENDEE; ROLE=CHAIR; STATUS=ACCEPTED : Mailto: Bdexample. com

ATTENDEE; TYPE=INDIVIDUAL:Mailto:C@example.com

ATTENDEE; TYPE=INDIVIDUAL:Mailto:D@example.com DTSTAMP : $19970611 \mathrm{~T} 190000 \mathrm{Z}$

DTSTART: $19970701 \mathrm{~T} 200000 \mathrm{Z}$

DTEND : $19970701 \mathrm{~T} 203000 \mathrm{Z}$

RRULE : FREQ=WEEKLY

SUMMARY:Phone Conference

UID : 123456 example.com 
SEQUENCE : 1

STATUS : CONF IRMED

END : VEVENT

END : VCALENDAR

4.3 Busy Time Examples

Busy time objects can be used in several ways. First, a CU may request busy time from another $C U$ for a specific range of time. That request can be answered with a busy time Reply. Additionally, a CU may simply publish their busy time for a given interval and point other CUs to the published location. The following examples outline both scenarios.

Individual "A" publishes busy time for one week.

BEGIN: VCALENDAR

PRODID:-//ACME/DesktopCalendar//EN

VERSION $: 2.0$

METHOD : PUBLISH

BEGIN : VFREEBUSY

DTSTAMP : $19980101 \mathrm{~T} 124100 \mathrm{Z}$

ORGANIZER:MAILTO:A@Example.com

DTSTART: $19980101 \mathrm{~T} 124200 \mathrm{Z}$

DTEND: $19980107 \mathrm{~T} 124200 \mathrm{Z}$

FREEBUSY : $19980101 \mathrm{~T} 180000 \mathrm{Z} / 19980101 \mathrm{~T} 190000 \mathrm{Z}$

FREEBUSY : $19980103 \mathrm{~T} 020000 \mathrm{Z} / 19980103 \mathrm{~T} 050000 \mathrm{Z}$

FREEBUSY: $19980107 \mathrm{~T} 020000 \mathrm{Z} / 19980107 \mathrm{~T} 050000 \mathrm{Z}$

FREEBUSY: $19980113 \mathrm{~T} 000000 \mathrm{z} / 19980113 \mathrm{~T} 010000 \mathrm{Z}$

FREEBUSY: $19980115 \mathrm{~T} 190000 \mathrm{Z} / 19980115 \mathrm{~T} 200000 \mathrm{Z}$

FREEBUSY : $19980115 \mathrm{~T} 220000 \mathrm{Z} / 19980115 \mathrm{~T} 230000 \mathrm{Z}$

FREEBUSY: $19980116 \mathrm{~T} 013000 \mathrm{Z} / 19980116 \mathrm{~T} 043000 \mathrm{Z}$

END:VFREEBUSY

END : VCALENDAR

Individual "A" requests busy time from individuals "B", "C". Individual "B" and "C" replies with busy time data to individual "A". The following table illustrates the sequence of messages that would be exchanged between these individuals. 


\begin{tabular}{|c|c|c|}
\hline Action & "Organizer" & Attendee \\
\hline $\begin{array}{l}\text { Initiate a busy } \\
\text { time request }\end{array}$ & $\begin{array}{l}\text { "A" sends "REQUEST" } \\
\text { message to "B" and } \\
\text { and "C" }\end{array}$ & \\
\hline $\begin{array}{l}\text { Reply to the "BUSY" } \\
\text { request with "BUSY" } \\
\text { time data }\end{array}$ & & $\begin{array}{l}\text { "B" sends a "REPLY" } \\
\text { message to "A" with } \\
\text { busy time data }\end{array}$ \\
\hline
\end{tabular}

\subsubsection{Request Busy Time}

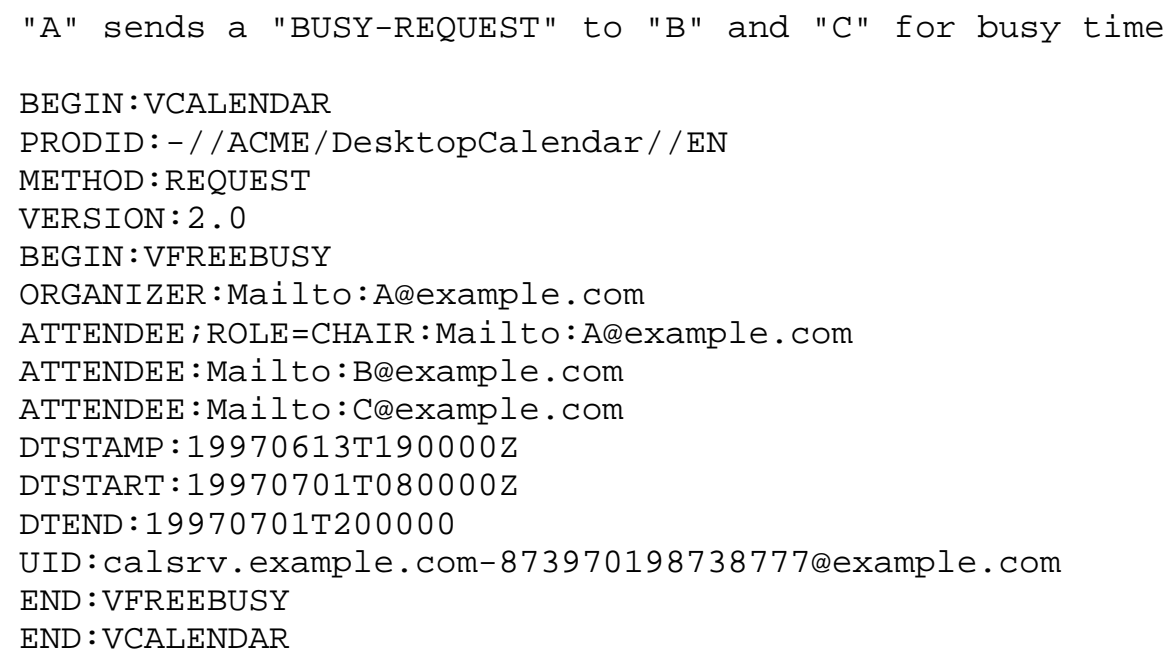

4.3.2 Reply To A Busy Time Request

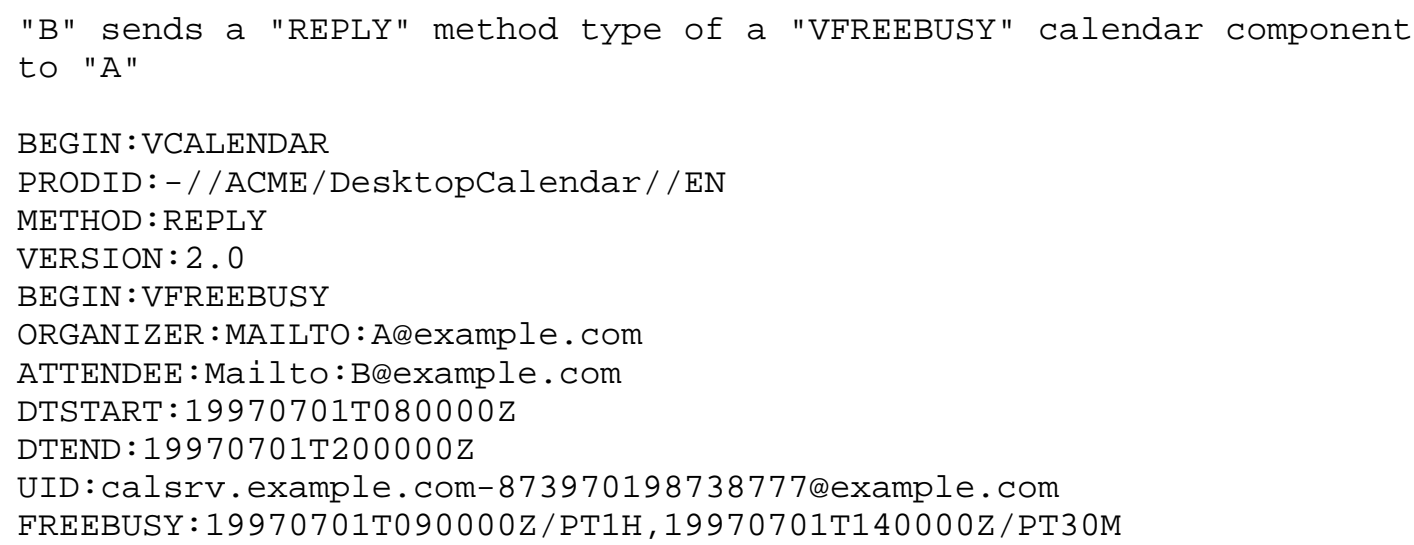

Silverberg, et. al. 
DTSTAMP : $19970613 \mathrm{~T} 190030 \mathrm{Z}$

END : VFREEBUSY

END : VCALENDAR

"B" is busy from 09:00 to 10:00 and from 14:00 to 14:30.

4.4 Recurring Event and Time Zone Examples

4.4.1 A Recurring Event Spanning Time Zones

This event describes a weekly phone conference. The "Attendees" are each in a different time zone.

BEGIN : VCALENDAR

PRODID : - / ACME / DesktopCalendar//EN

METHOD : REQUEST

VERSION : 2 . 0

BEGIN : VTIMEZONE

TZID:America-SanJose

TZURL:http://zones.stds_r_us.net/tz/America-SanJose

BEGIN : STANDARD

DTSTART : $19671029 T 020000$

RRULE : $F R E Q=Y E A R L Y ; B Y D A Y=-1 S U$; $B Y M O N T H=10$

TZOFFSETFROM : -0700

TZOFFSETTO: -0800

TZNAME :PST

END : STANDARD

BEGIN : DAYLIGHT

DTSTART : $19870405 T 020000$

RRULE : $F R E Q=Y E A R L Y ; B Y D A Y=1 S U$; $B Y M O N T H=4$

TZOFFSETFROM: -0800

TZOFFSETTO: -0700

TZNAME :PDT

END : DAYLIGHT

END : VTIMEZONE

BEGIN : VEVENT

ORGANIZER:Mailto:A@example.com

ATTENDEE; ROLE=CHAIR; PARTSTAT=ACCEPTED; TYPE=INDIVIDUAL : Adexample.COM

ATTENDEE; RSVP=TRUE; TYPE=INDIVIDUAL : B@example.fr

ATTENDEE; RSVP=TRUE; TYPE=INDIVIDUAL : c lexample.jp

DTSTAMP : $19970613 \mathrm{~T} 190030 \mathrm{Z}$

DTSTART; TZID=America-SanJose: $19970701 \mathrm{~T} 140000$

DTEND; TZID=America-SanJose: $19970701 \mathrm{~T} 150000$

RRULE : $F R E Q=W E E K L Y ; I N T E R V A L=20 ; W K S T=S U ; B Y D A Y=T U$

RDATE; TZID=America-SanJose: $19970910 \mathrm{~T} 140000$

EXDATE; TZID=America-SanJose:19970909T140000

EXDATE; TZID=America-SanJose: $19971028 \mathrm{~T} 140000$

SUMMARY:Weekly Phone Conference 
UID:calsrv.example.com-873970198738777@example.com

SEQUENCE : 0

STATUS : CONF IRMED

END : VEVENT

END : VCALENDAR

The first two components of this icalendar object are the time zone components. The "DTSTART" date coincides with the first instance of the RRULE property.

The recurring meeting is defined in a particular time zone, presumably that of the originator. The client for each "Attendee" has the responsibility of determining the recurrence time in the

"Attendee's" time zone.

The repeating event starts on Tuesday, July 1, 1997 at 2:00pm PDT. "Attendee" B@example.fr is in France where the local time on this date is 9 hours ahead of PDT or 23:00. "Attendee" CQexample.jp is in Japan where local time is 8 hours ahead of UTC or Wednesday, July 2 at 06:00. The event repeats weekly on Tuesdays (in PST/PDT). The "RRULE" property results in 20 instances. The last instance falls on Tuesday, November 11, 1997 2:00pm PDT. The "RDATE" property adds another instance: WED, 10-SEP-1997 2:00 PM PST.

There are two exceptions to this recurring appointment. The first one is:

TUE, 09-SEP-1997 23:00 GMT

TUE, 09-SEP-1997 14:00 PDT

WED, 10-SEP-1997 06:00 JST

and the second is:

TUE, 28-OCT-1997 23:00 GMT

TUE, 28-OCT-1997 14:00 PST

WED, 29-OCT-1997 06:00 JST

\subsubsection{Modify A Recurring Instance}

In this example the "Organizer" issues a recurring meeting. Later the "Organizer" changes an instance of the event by changing the "DTSTART" property. Note the use of "RECURRENCE-ID" property and "SEQUENCE" property in the second request.

Original Request:

BEGIN : VCALENDAR

METHOD : REQUEST

Silverberg, et. al.

Standards Track

[Page 79] 


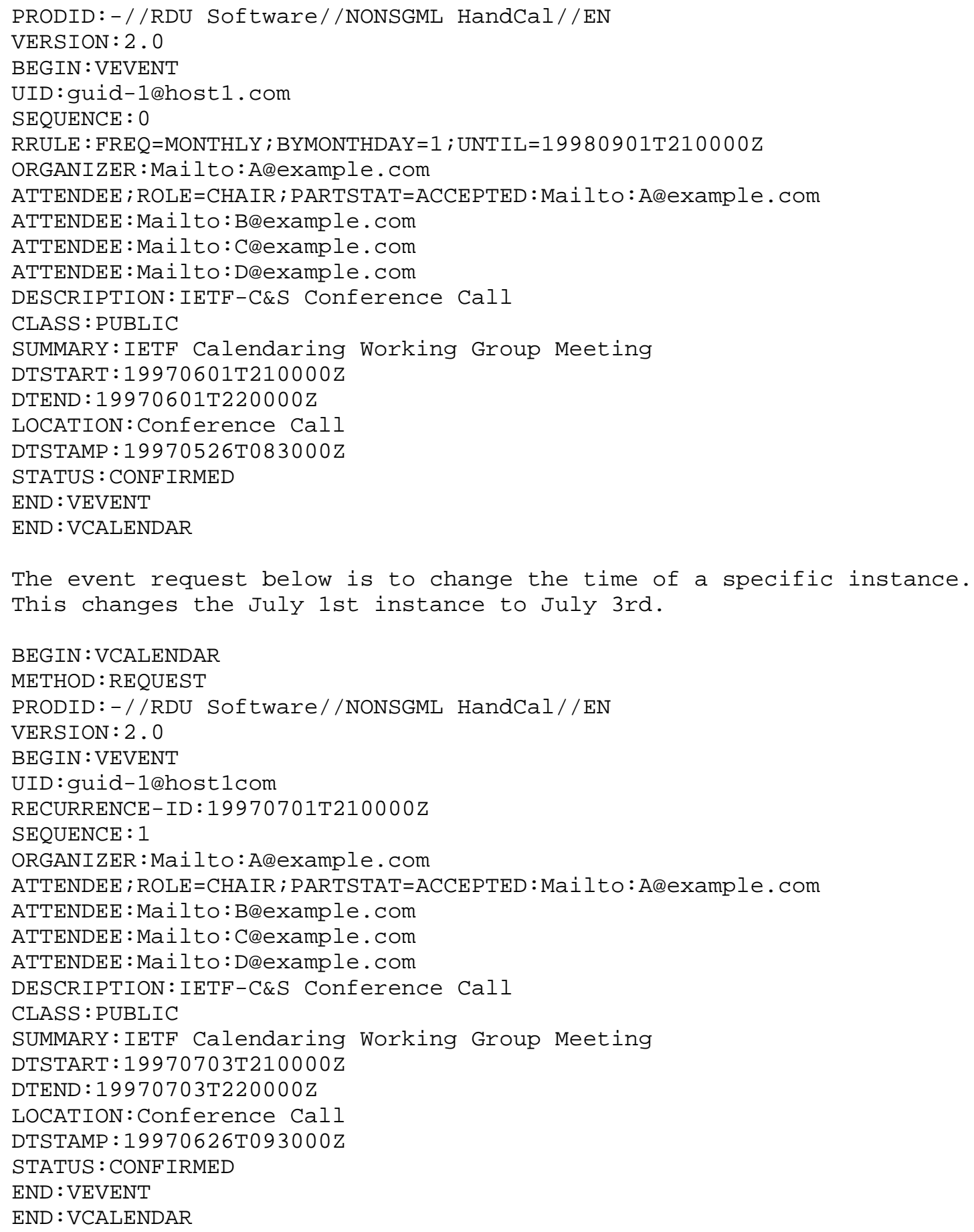


4.4.3 Cancel an Instance

In this example the "Organizer" of a recurring event deletes the August 1st instance.

BEGIN : VCALENDAR

METHOD : CANCEL

PRODID:-//RDU Software//NONSGML HandCal//EN

VERSION $: 2.0$

BEGIN : VEVENT

UID : guid-1@host1.com

ORGANIZER:Mailto:A@example.com

ATTENDEE; ROLE=CHAIR; PARTSTAT=ACCEPTED : Mailto: Alexample.com

ATTENDEE:Mailto:B@example.com

ATTENDEE:Mailto:C@example.com

ATTENDEE:Mailto:D@example.com

RECURRENCE-ID:19970801T210000Z

SEQUENCE : 2

STATUS : CANCELLED

DTSTAMP $: 19970721 \mathrm{~T} 093000 \mathrm{Z}$

END:VEVENT

END : VCALENDAR

4.4.4 Cancel Recurring Event

In this example the "Organizer" wishes to cancel the entire recurring event and any exceptions.

BEGIN : VCALENDAR

METHOD : CANCEL

PRODID:-//RDU Software//NONSGML HandCal//EN

VERSION $: 2.0$

BEGIN: VEVENT

UID : guid-1@host1.com

ORGANIZER:Mailto:A@example.com

ATTENDEE; ROLE=CHAIR; PARTSTAT=ACCEPTED :Mailto: Adexample.com

ATTENDEE:Mailto:Bdexample.com

ATTENDEE:Mailto:C@example.com

ATTENDEE:Mailto:D@example.com

DTSTAMP : $19970721 \mathrm{~T} 103000 \mathrm{z}$

STATUS : CANCELLED

SEQUENCE : 3

END :VEVENT

END : VCALENDAR 


\subsubsection{Change All Future Instances}

This example changes the meeting location from a conference call to Seattle starting september 1 and extends to all future instances.

BEGIN : VCALENDAR

METHOD : REQUEST

PRODID:-//RDU Software//NONSGML HandCal//EN

VERSION $: 2.0$

BEGIN : VEVENT

UID : guid-1ehost 1 . com

RECURRENCE-ID; THISANDFUTURE : $19970901 \mathrm{~T} 210000 \mathrm{Z}$

SEQUENCE : 3

ORGANIZER:Mailto:A@example.com

ATTENDEE; ROLE=CHAIR; PARTSTAT=ACCEPTED : Mailto: A@example.com

ATTENDEE; RSVP=TRUE : Mailto:B@example.com

ATTENDEE; RSVP=TRUE: Mailto: C@example.com

ATTENDEE; RSVP=TRUE: Mailto:D@example.com

DESCRIPTION:IETF-C\&S Discussion

CLASS : PUBLIC

SUMMARY:IETF Calendaring Working Group Meeting

DTSTART : $19970901 \mathrm{~T} 210000 \mathrm{Z}$

DTEND : $19970901 \mathrm{~T} 220000 \mathrm{Z}$

LOCATION:Building 32, Microsoft, Seattle, WA

DTSTAMP : $19970526 \mathrm{~T} 083000 \mathrm{Z}$

STATUS : CONF IRMED

END : VEVENT

END : VCALENDAR

4.4.6 Add A New Instance To A Recurring Event

This example adds a one-time additional instance to the recurring event. "Organizer" adds a second July meeting on the 15th.

BEGIN : VCALENDAR

METHOD : ADD

PRODID:-//RDU Software//NONSGML HandCal//EN

VERSION : 2 . 0

BEGIN : VEVENT

UID : 123456789 @host 1 . com

SEQUENCE : 4

ORGANIZER:Mailto:A@example.com

ATTENDEE; ROLE=CHAIR; PARTSTAT=ACCEPTED : Mailto: A@example. com

ATTENDEE; RSVP=TRUE:Mailto:B@example.com

ATTENDEE; RSVP=TRUE:Mailto: C@example.com

ATTENDEE; RSVP=TRUE : Mailto:D@example.com DESCRIPTION:IETF-C\&S Conference Call

CLASS : PUBLIC 
SUMMARY:IETF Calendaring Working Group Meeting

DTSTART : $19970715 \mathrm{~T} 210000 \mathrm{Z}$

DTEND : $19970715 \mathrm{~T} 220000 \mathrm{Z}$

LOCATION:Conference Call

DTSTAMP : $19970629 \mathrm{~T} 093000 \mathrm{Z}$

STATUS : CONF IRMED

END : VEVENT

END : VCALENDAR

4.4.7 Add A New Series of Instances To A Recurring Event

The scenario for this example involves an ongoing meeting, originally set up to occur every Tuesday. The "Organizer" later decides that the meetings need to be on Tuesdays and Thursdays, but does not want to reschedule the entire meeting or lose any of the per-instance information already collected.

The original event:

BEGIN : VCALENDAR

METHOD : REQUEST

PRODID:-//RDU Software//NONSGML HandCal//EN

VERSION : 2 . 0

BEGIN : VEVENT

UID : 123456789 @host 1 . com

SEQUENCE : 0

RRULE $:$ WKST $=S U ; B Y D A Y=T U ; F R E Q=W E E K L Y$

ORGANIZER:Mailto:A@example.com

ATTENDEE; ROLE=CHAIR; PARTSTAT=ACCEPTED : Mailto:A@example.com

ATTENDEE; RSVP=TRUE:Mailto:B@example.com

SUMMARY:Review Accounts

DTSTART : $19980303 \mathrm{~T} 210000 \mathrm{Z}$

DTEND : $19980303 \mathrm{~T} 220000 \mathrm{Z}$

LOCATION:The White Room

DTSTAMP : 19980301 T093000Z

STATUS : CONF IRMED

END : VEVENT

END : VCALENDAR

Assume that many other updates happen to this event and that a lot of instance-specific information exists in the recurring series. The "SEQUENCE" property value is 7 for the next update. Now the

"Organizer" wants to add Thursdays to the event:

BEGIN : VCALENDAR

METHOD : ADD

PRODID:-//RDU Software//NONSGML HandCal//EN

VERSION $: 2.0$ 


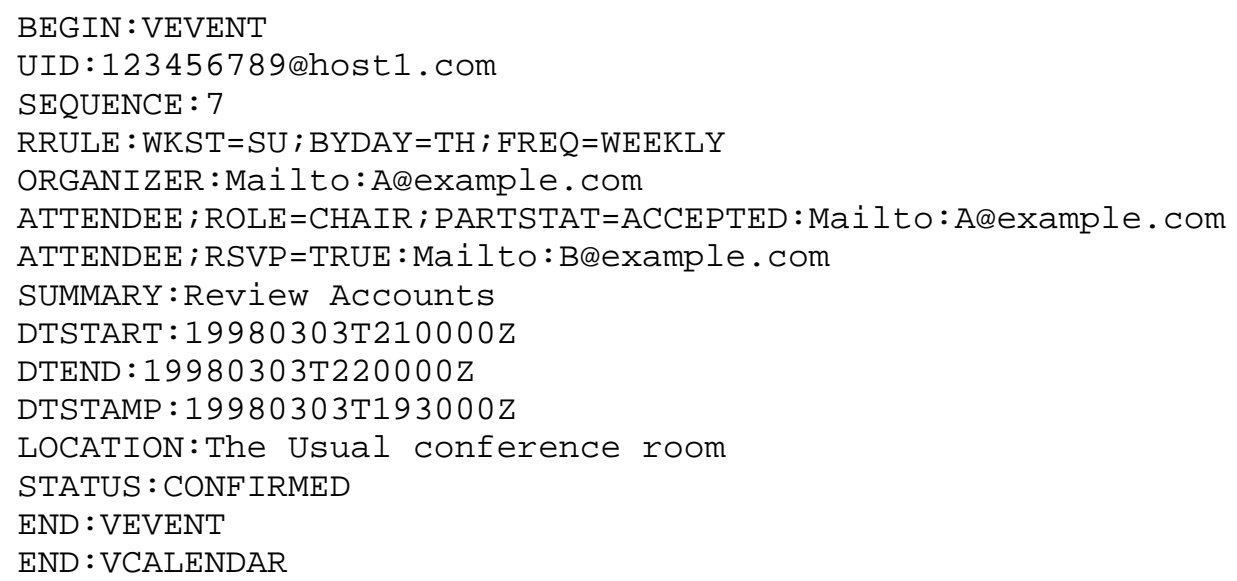




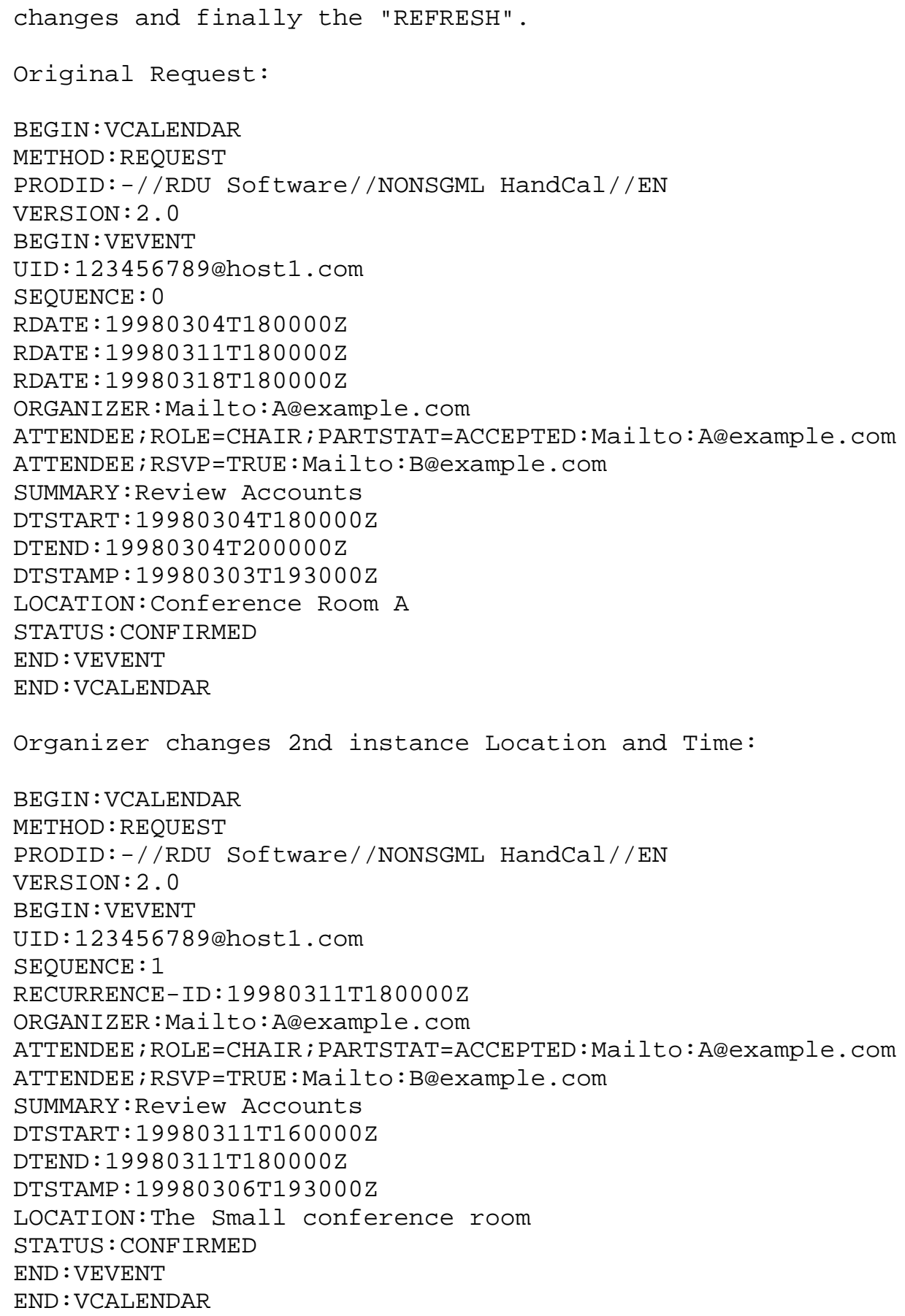




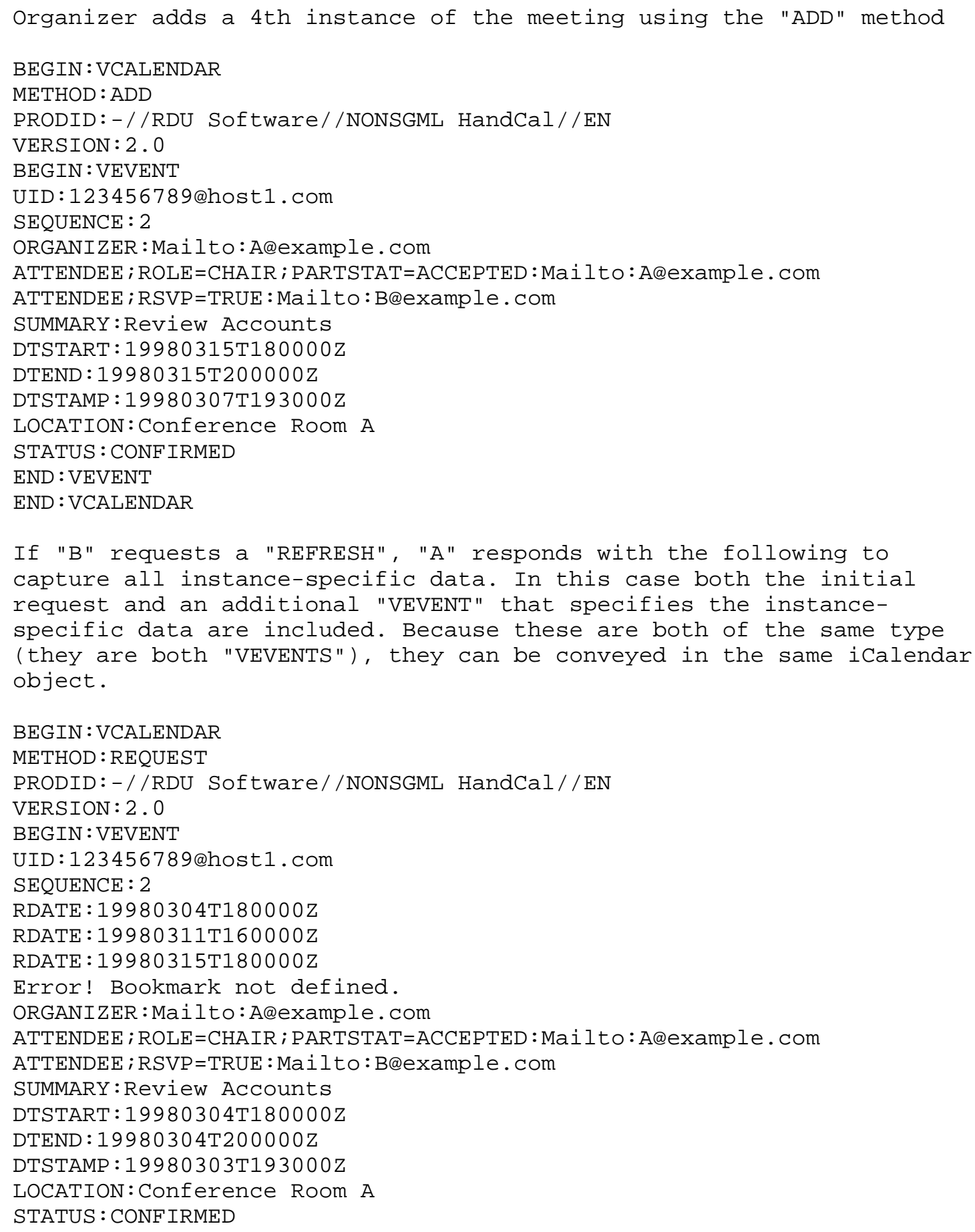


END : VEVENT

BEGIN : VEVENT

Error! Bookmark not defined. SEQUENCE : 2

RECURRENCE-ID:19980311T160000Z

Error! Bookmark not defined.

ATTENDEE; ROLE=CHAIR; Error! Bookmark not defined. ATTENDEE; Error! Bookmark not defined. SUMMARY:Review Accounts DTSTART: $19980311 \mathrm{~T} 160000 \mathrm{Z}$ DTEND: $19980304 \mathrm{~T} 180000 \mathrm{Z}$ DTSTAMP $: 19980306 \mathrm{~T} 193000 \mathrm{z}$ LOCATION:The Small conference room STATUS : CONF IRMED

END : VEVENT

END : VCALENDAR

4.4.8 Counter An Instance of A Recurring Event

In this example one of the "Attendees" counters the "DTSTART" property of the proposed second July meeting.

BEGIN : VCALENDAR

METHOD : COUNTER

PRODID:-//RDU Software//NONSGML HandCal//EN

VERSION $: 2.0$

BEGIN: VEVENT

UID: guid-1@host 1.com

RECURRENCE-ID:19970715T210000Z

SEQUENCE $: 4$

ORGANI ZER:Mailto:A@example.com

ATTENDEE; ROLE=CHAIR; RSVP=TRUE : Mailto: A@example.com

ATTENDEE; RSVP=TRUE: Mailto: B eexample.com

ATTENDEE; RSVP=TRUE: Mailto: C@example.com

ATTENDEE; RSVP=TRUE: Mailto:D@example.com DESCRIPTION:IETF-C\&S Conference Call

CLASS : PUBLIC

SUMMARY:IETF Calendaring Working Group Meeting DTSTART:19970715T220000z

DTEND: $19970715 \mathrm{~T} 230000 \mathrm{Z}$

LOCATION:Conference Call

COMMENT:May we bump this by an hour? I have a conflict DTSTAMP : 19970629 T094000z

END : VEVENT

END : VCALENDAR 
4.4.9 Error Reply To A Request

The following example illustrates a scenario where a meeting is proposed containing an unsupported property and a bad property.

Original Request

BEGIN : VCALENDAR

METHOD : REQUEST

PRODID:-//RDU Software//NONSGML HandCal//EN

VERSION : 2 . 0

BEGIN : VEVENT

UID : guid-1@host 1. com

SEQUENCE : 0

RRULE : $F R E Q=M O N T H L Y ; B Y M O N T H D A Y=1$

ORGANIZER:Mailto:A@example.com

ATTENDEE; ROLE=CHAIR: Mailto: A@example.com

ATTENDEE; RSVP=TRUE : Mailto:B@example.com

ATTENDEE; RSVP=TRUE : Mailto: C@example.com

ATTENDEE; RSVP=TRUE : Mailto:D@example.com DESCRIPTION:IETF-C\&S Conference Call

CLASS : PUBLIC

SUMMARY:IETF Calendaring Working Group Meeting

DTSTART : $19970601 \mathrm{~T} 210000 \mathrm{Z}$

DTEND : $19970601 \mathrm{~T} 220000 \mathrm{Z}$

DTSTAMP : $19970602 \mathrm{~T} 094000 \mathrm{Z}$

LOCATION:Conference Call

STATUS : CONF IRMED

FOO:BAR

END : VEVENT

END : VCALENDAR

Response from "B" to indicate that RRULE is not supported and an unrecognized property was encountered

BEGIN : VCALENDAR

PRODID:-//RDU Software//NONSGML HandCal//EN

METHOD : REP LY

VERSION : 2 . 0

BEGIN : VEVENT

ORGANIZER:Mailto:A@example.com

ATTENDEE:Mailto:B@example.com

REQUEST-STATUS:2.8; Repeating event ignored. Scheduled as a single event; RRULE

REQUEST-STATUS:3.0; Invalid Property Name; FOO

UID : guid-1@host 1 . com

SEQUENCE : 0

DTSTAMP : 19970603 T094000Z 
END : VEVENT

END : VCALENDAR

\subsection{Group To-do Examples}

Individual "A" creates a group task in which individuals "A", "B", "C" and "D" will participate. Individual "B" confirms acceptance of the task. Individual "C" declines the task. Individual "D" tentatively accepts the task. The following table illustrates the sequence of messages that would be exchanged between these individuals. Individual "A" then issues a "REQUEST" method to obtain the status of the to-do from each participant. The response indicates the individual "Attendee's" completion status. The table below illustrates the message flow.

\begin{tabular}{|c|c|c|}
\hline Action & "Organizer" & Attendee \\
\hline $\begin{array}{l}\text { Initiate a to-do } \\
\text { request }\end{array}$ & $\begin{array}{l}\text { "A" sends a REQUEST } \\
\text { message to "B", "C", } \\
\text { and "D" }\end{array}$ & \\
\hline $\begin{array}{l}\text { Accept the to-do } \\
\text { request }\end{array}$ & & $\begin{array}{l}\text { "B" sends a "REPLY" } \\
\text { message to "A" with its } \\
\text { "partstat" paramater } \\
\text { set to "accepted". }\end{array}$ \\
\hline $\begin{array}{l}\text { Decline the to-do } \\
\text { request }\end{array}$ & & $\begin{array}{l}\text { "C" sends a REPLY } \\
\text { message to "A" with its } \\
\text { "partstat" parameter } \\
\text { set to "declined". }\end{array}$ \\
\hline $\begin{array}{l}\text { Tentatively accept } \\
\text { the to-do request }\end{array}$ & & $\begin{array}{l}\text { "D" sends a REPLY } \\
\text { message to "A" with its } \\
\text { "partstat" parameter } \\
\text { set to "tentative". }\end{array}$ \\
\hline $\begin{array}{l}\text { Check attendee } \\
\text { completion status }\end{array}$ & $\begin{array}{l}\text { "A" sends a REQUEST } \\
\text { message to "B" and } \\
\text { "D" with current } \\
\text { information. }\end{array}$ & \\
\hline $\begin{array}{l}\text { Attendee indicates } \\
\text { percent complete }\end{array}$ & & $\begin{array}{l}\text { "B" sends a "REPLY" } \\
\text { message indicating } \\
\text { percent complete }\end{array}$ \\
\hline
\end{tabular}




$\mid$\begin{tabular}{l|l|}
$\begin{array}{l}\text { Attendee indicates } \\
\text { completion }\end{array}$ & $\begin{array}{l}\text { "D" sends a "REPLY" } \\
\text { message indicating } \\
\text { completion }\end{array}$
\end{tabular}

\subsubsection{A VTODO Request}

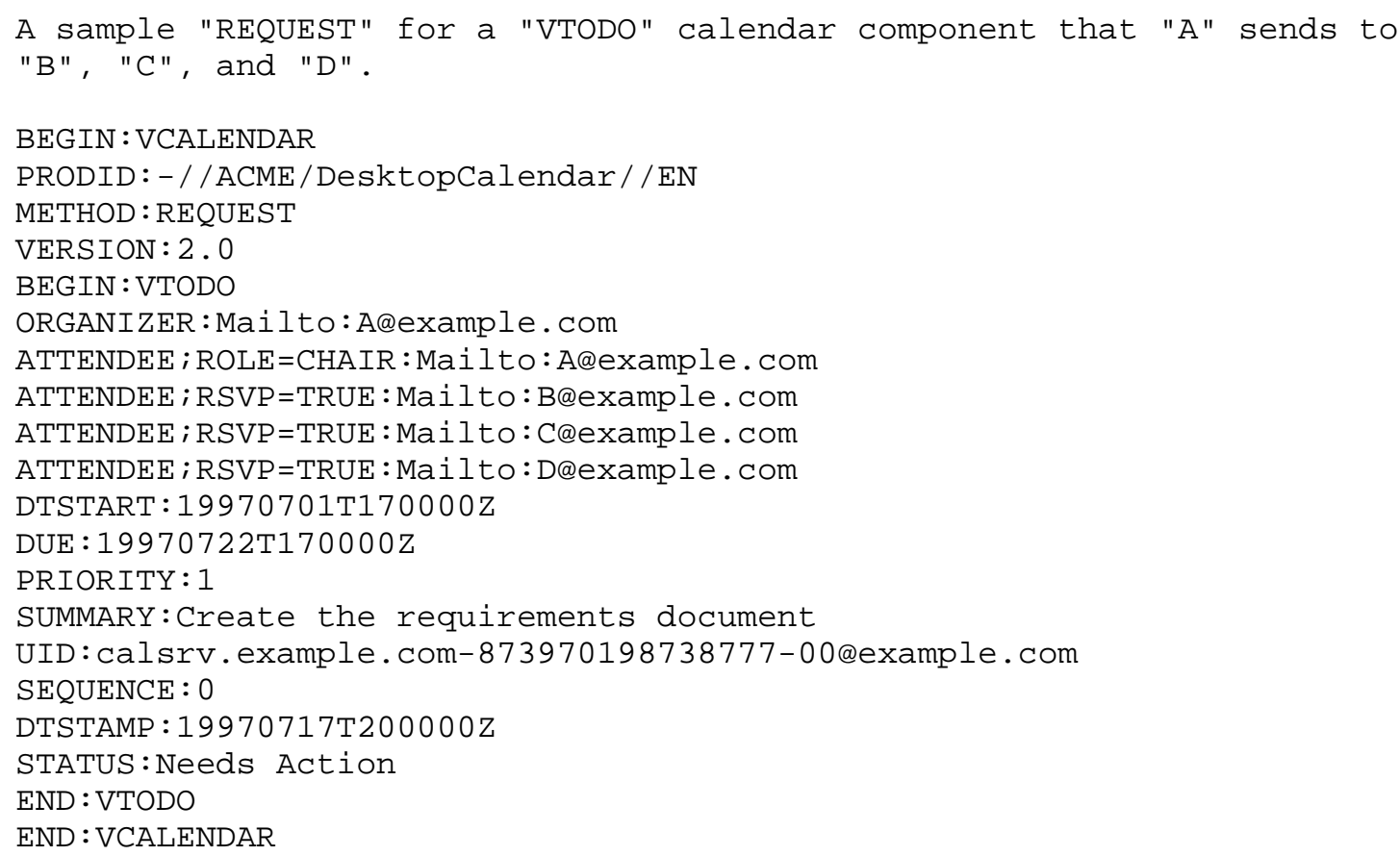


END : VTODO

END : VCALENDAR

"B" could have declined the TODO or indicated tentative acceptance by setting the "partstat" property parameter sequence to "declined" or "tentative", respectively.

4.5.3 A VTODO Request for Updated Status

"A" requests status from all "Attendees".

BEGIN : VCALENDAR

PRODID :-//ACME/DesktopCalendar//EN

METHOD : REQUEST

VERSION : 2.0

BEGIN : VTODO

ORGANIZER:Mailto:A@example.com

ATTENDEE; ROLE=CHAIR:Mailto:A@example.com

ATTENDEE; RSVP=TRUE; TYPE=INDIVIDUAL : Mail to: B@example.com

ATTENDEE; RSVP=TRUE; TYPE=INDIVIDUAL : Mail to: D @example. com

UID:calsrv.example.com-873970198738777-00@example.com

SUMMARY:Create the requirements document

PRIORITY : 1

SEQUENCE : 0

STATUS : IN-PROCESS

DTSTART : $19970701 \mathrm{~T} 170000 \mathrm{Z}$

DTSTAMP : $19970717 \mathrm{~T} 230000 \mathrm{Z}$

END : VTODO

END : VCALENDAR

4.5.4 A Reply: Percent-Complete

A reply indicating the task being worked on and that "B" is 75\% complete with "B's" part of the assignment.

BEGIN : VCALENDAR

PRODID :-//ACME/DesktopCalendar//EN

METHOD : REPLY

VERSION : 2 . 0

BEGIN : VTODO

ORGANIZER:MAILTO:A@example.com

ATTENDEE; PARTSTAT=IN-PROCESS:Mailto:B@example.com PERCENT-COMP LETE : 75

UID : calsrv.example.com-873970198738777-00@example.com

DTSTAMP : 19970717 T233000Z

SEQUENCE : 0

END : VTODO

END : VCALENDAR

Silverberg, et. al

Standards Track

[Page 91] 
4.5.5 A Reply: Completed

A reply indicating that "D" completed "D's" part of the assignment. BEGIN : VCALENDAR

PRODID:-//ACME/DesktopCalendar//EN

METHOD : REPLY

VERSION $: 2.0$

BEGIN : VTODO

ORGANIZER:MAILTO:A@example.com

ATTENDEE; PARTSTAT=COMPLETED:Mailto:D@example.com

UID:calsrv.example.com-873970198738777-00@example.com

DTSTAMP : 19970717 T233000Z

SEQUENCE : 0

END : VTODO

END : VCALENDAR

4.5.6 An Updated VTODO Request

Organizer "A" resends the "VTODO" calendar component. "A" sets the overall completion for the to-do at $40 \%$.

BEGIN : VCALENDAR

PRODID : - / /ACME/DesktopCalendar//EN

METHOD : REQUEST

VERSION : 2 . 0

BEGIN : VTODO

ORGANIZER:Mailto:A@example.com

ATTENDEE; ROLE=CHAIR; PARTSTAT=ACCEPTED : Mailto: A@example. com ATTENDEE; PARTSTAT=ACCEPTED; TYPE=INDIVIDUAL : Mail to : B@example.com ATTENDEE; PARTSTAT=IN-PROCESS; TYPE=INDIVIDUAL : Mail to:D example.com DTSTART : $19970701 \mathrm{~T} 170000 \mathrm{Z}$

DUE : $19970722 \mathrm{~T} 170000 \mathrm{Z}$

PRIORITY : 1

SUMMARY:Create the requirements document

UID:calsrv.example.com-873970198738777-00@example.com SEQUENCE : 1

DTSTAMP : $19970718 \mathrm{~T} 100000 \mathrm{Z}$

STATUS : IN-PROGRESS

PERCENT-COMP LETE : 40

END : VTODO

END : VCALENDAR

4.5.7 Recurring VTODOs

The following examples relate to recurring "VTODo" calendar components. 
4.5.7.1 Request for a Recurring VTODO

In this example "A" sends a recurring "VTODO" calendar component to "B" and "D".

BEGIN : VCALENDAR

PRODID:-//ACME/DesktopCalendar//EN

METHOD : REQUEST

VERSION $: 2.0$

BEGIN : VTODO

ORGANIZER:Mailto:A@example.com

ATTENDEE; ROLE=CHAIR:Mailto: Adexample.com

ATTENDEE; RSVP=TRUE; TYPE=INDIVIDUAL : Mail to:B@example.com

ATTENDEE; RSVP=TRUE; TYPE $=I N D I V I D U A L:$ Mail to $:$ D@example. $\mathrm{com}$

RRULE : $F R E Q=M O N T H L Y ; C O U N T=10 ; B Y D A Y=1 F R$

DTSTART $: 19980101 \mathrm{~T} 100000-0700$

DUE:19980103T100000-0700

SUMMARY:Send Status Reports to Area Managers

UID:calsrv.example.com-873970198738777-00@example.com

SEQUENCE : 0

DTSTAMP : 19970717 T200000Z

STATUS:NEEDS ACTION

PRIORITY : 1

END : VTODO

END : VCALENDAR

4.5.7.2 Calculating due dates in recurring VTODOs

The due date in a recurring "VTODO" calendar component is either a fixed interval specified in the "REQUEST" method or specified using the "RECURRENCE-ID" property. The former is calculated by applying the difference between "DTSTART" and "DUE" properties and applying it to each of the start of each recurring instance. Hence, if the initial "VTODO" calendar component specifies a "DTSTART" property value of "19970701T190000z" and a "DUE" property value of "19970801T190000z" the interval of one day which is applied to each recurring instance of the "VTODO" calendar component to determine the "DUE" date of the instance.

4.5.7.3 Replying to an instance of a recurring VTODO

In this example "B" updates "A" on a single instance of the "VTODO" calendar component.

BEGIN: VCALENDAR

PRODID:-//ACME/DesktopCalendar//EN

METHOD : REPLY

VERSION $: 2.0$ 


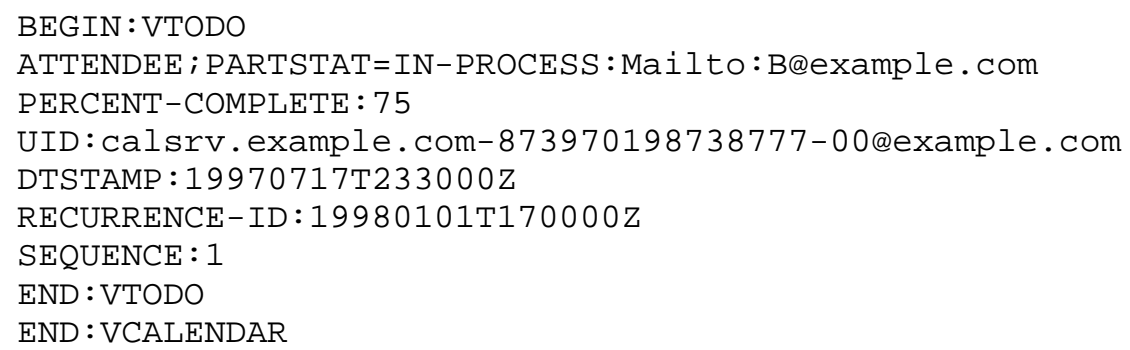

4.7 Other Examples

4.7.1 Event Refresh

Refresh the event with "UID" property value of "guid-112345 @host 1 .com" :

BEGIN : VCALENDAR

PRODID:-//RDU Software//NONSGML HandCal//EN

METHOD : REFRESH

VERSION : 2 . 0

BEGIN : VEVENT

ORGANIZER:Mailto:A@example.com

ATTENDEE; ROLE=CHAIR;PARTSTAT=ACCEPTED : Mailto: A@example.com ATTENDEE:Mailto:B@example.com ATTENDEE:Mailto:C@example.com 
ATTENDEE:Mailto:D@example.com

UID: guid-1-12345@host1.com

DTSTAMP : 19970603 T094000

END : VEVENT

END : VCALENDAR

\subsubsection{Bad RECURRENCE-ID}

Component instances are identified by the combination of "UID", "RECURRENCE-ID", and "SEQUENCE". When an "Organizer" sends a request to an "Attendee", there are three cases in which an instance cannot be found. They are:

1. The component with the referenced "UID" and "RECURRENCE-ID" has been found but the "SEQUENCE" number in the calendar store does not match that of the ITIP message.

2. The component with the referenced "UID" has been found, the "SEQUENCE" numbers match, but the "RECURRENCE-ID" cannot be found.

3. The "UID" and "SEQUENCE" numbers are found but the CUA does not support recurrences.

In case (1), two things can happen. If the "SEQUENCE" number of the "Attendee's" instance is larger than that in the "Organizer's" message then the "Attendee" is receiving an out-of-sequence message and MUST ignore it. If the "SEQUENCE" number of the "Attendee's" instance is smaller, then the "Organizer" is sending out a newer version of the component and the "Attendee's" version needs to be updated. Since one or more updates have been missed, the "Attendee" SHOULD send a "REFRESH" message to the "Organizer" to get an updated version of the event.

In case (2), something has gone wrong. Both the "Organizer" and the "Attendee" should have the same instances, but the "Attendee" does not have the referenced instance. In this case the "Attendee" SHOULD send a "REFRESH" to the "Organizer" to get an updated version of the event.

In case (3), the limitations of the "Attendee's" CUA makes it impossible to match an instance other than the single instance scheduled. In this case, the "Attendee" need not send a "REFRESH" to the "Organizer".

The example below shows a sequence in which an "Attendee" sends a "REFRESH" to the "Organizer". 


\begin{tabular}{|c|c|c|}
\hline Action & "Organizer" & Attendee \\
\hline $\begin{array}{l}\text { Update an instance } \\
\text { request }\end{array}$ & $\begin{array}{l}\text { "A" sends "REQUEST" } \\
\text { message to "B" }\end{array}$ & \\
\hline $\begin{array}{l}\text { Attendee requests } \\
\text { refresh because } \\
\text { "RECURRENCE-ID" was } \\
\text { not found }\end{array}$ & & $\begin{array}{l}\text { "B" sends a "REFRESH" } \\
\text { message to "A" }\end{array}$ \\
\hline $\begin{array}{l}\text { Refresh the entire } \\
\text { Event }\end{array}$ & $\begin{array}{l}\text { "A" sends the } \\
\text { latest copy of the } \\
\text { Event to "B" }\end{array}$ & \\
\hline $\begin{array}{l}\text { Attendee handles } \\
\text { the request and } \\
\text { updates the } \\
\text { instance }\end{array}$ & & $\begin{array}{l}\text { "B" updates to the } \\
\text { latest copy of the } \\
\text { meeting. }\end{array}$ \\
\hline
\end{tabular}

Request from "A":

BEGIN : VCALENDAR

METHOD : REQUEST

PRODID:-//RDU Software//NONSGML HandCal//EN

VERSION : 2 . 0

BEGIN : VEVENT

UID : acme-12345@host 1 . com

SEQUENCE : 3

RRULE : FREQ=WEEKLY

RDATE; VALUE=PERIOD : $19970819 \mathrm{~T} 210000 \mathrm{Z} / 199700819 \mathrm{~T} 220000 \mathrm{Z}$

ORGANIZER:Mailto:A@example.com

ATTENDEE; ROLE=CHAIR; PARTSTAT=ACCEPTED : Mailto: A@example. com

ATTENDEE:Mailto:B@example.com

DESCRIPTION:IETF-C\&S Conference Call

SUMMARY:IETF Calendaring Working Group Meeting

DTSTART : $19970801 \mathrm{~T} 210000 \mathrm{Z}$

DTEND : $19970801 \mathrm{~T} 220000 \mathrm{Z}$

RECURRENCE-ID:19970809T210000Z

DTSTAMP : 19970726 T083000

STATUS : CONF IRMED

END : VEVENT

END : VCALENDAR

"B" has the event with "UID" property "acme-12345@host1.com" but "B's" "SEQUENCE" property value is "1" and the event does not have an instance at the specified recurrence time. This means that "B" has 


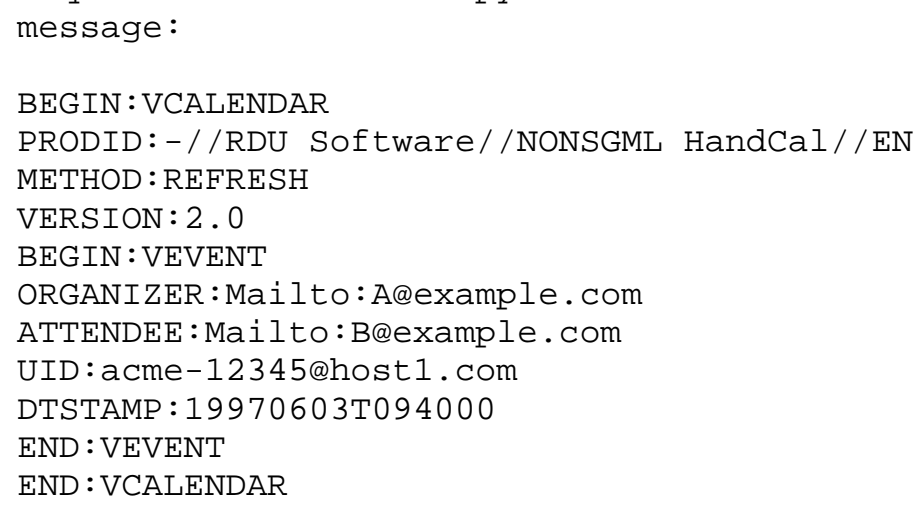

Method

PUBLISH

REQUEST

REPLY

$\mathrm{ADD}$

CANCEL

REFRESH

COUNTER

DECLINECOUNTER
Fallback

Required

PUBLISH

Required

Required

Required

Required

Reply with Not Supported

Required if EVENT-COUNTER is implemented; otherwise reply with Not Supported

Fallback

Ignore; assume GREGORIAN

Ignore

Required as described in the Method list above

Ignore 


\begin{tabular}{|c|c|}
\hline $\begin{array}{l}\text { Event-Related } \\
\text { Components }\end{array}$ & Fallback \\
\hline VALARM & Reply with Not Supported \\
\hline VTIMEZONE & Required if any DateTime value refers to a time zone. \\
\hline \multicolumn{2}{|l|}{ Component } \\
\hline Property & Fallback \\
\hline ATTACH & Ignore \\
\hline ATTENDEE & $\begin{array}{l}\text { Required if EVENT-REQUEST is not implemented; } \\
\text { otherwise reply with Not supported }\end{array}$ \\
\hline CATEGORIES & Ignore \\
\hline CLASS & Ignore \\
\hline COMMENT & Ignore \\
\hline COMP LETED & Ignore \\
\hline nCONTACT & Ignore \\
\hline CREATED & Ignore \\
\hline DESCRIPTION & Required \\
\hline DURATION & Reply with Not Supported \\
\hline DTSTAMP & Required \\
\hline DTSTART & Required \\
\hline DTEND & Required \\
\hline EXDATE & Ignore \\
\hline EXRULE & $\begin{array}{l}\text { Ignore Reply with Not supported. If implemented, } \\
\text { VTIMEZONE MUST also be implemented. }\end{array}$ \\
\hline GEO & Ignore \\
\hline LAST-MODIFIED & Ignore \\
\hline LOCATION & Required \\
\hline ORGANIZER & Ignore \\
\hline PRIORITY & Ignore \\
\hline RELATED-TO & Ignore \\
\hline RDATE & Ignore \\
\hline RRULE & $\begin{array}{l}\text { Ignore. The first instance occurs on the DTStart } \\
\text { property. If implemented, VTIMEZONE MUST also be } \\
\text { implemented. }\end{array}$ \\
\hline RECURRENCE-ID & Required if RRULE is implemented; otherwise Ignore \\
\hline REQUEST-STATUS & Required \\
\hline RESOURCES & Ignore \\
\hline SEQUENCE & Required \\
\hline STATUS & Ignore \\
\hline SUMMARY & Ignore \\
\hline TRANSP & Required if FREEBUSY is implemented; otherwise Ignore \\
\hline URL & Ignore \\
\hline UID & Required \\
\hline$X-$ & Ignore \\
\hline
\end{tabular}

Silverberg, et. al. Standards Track [Page 98] 


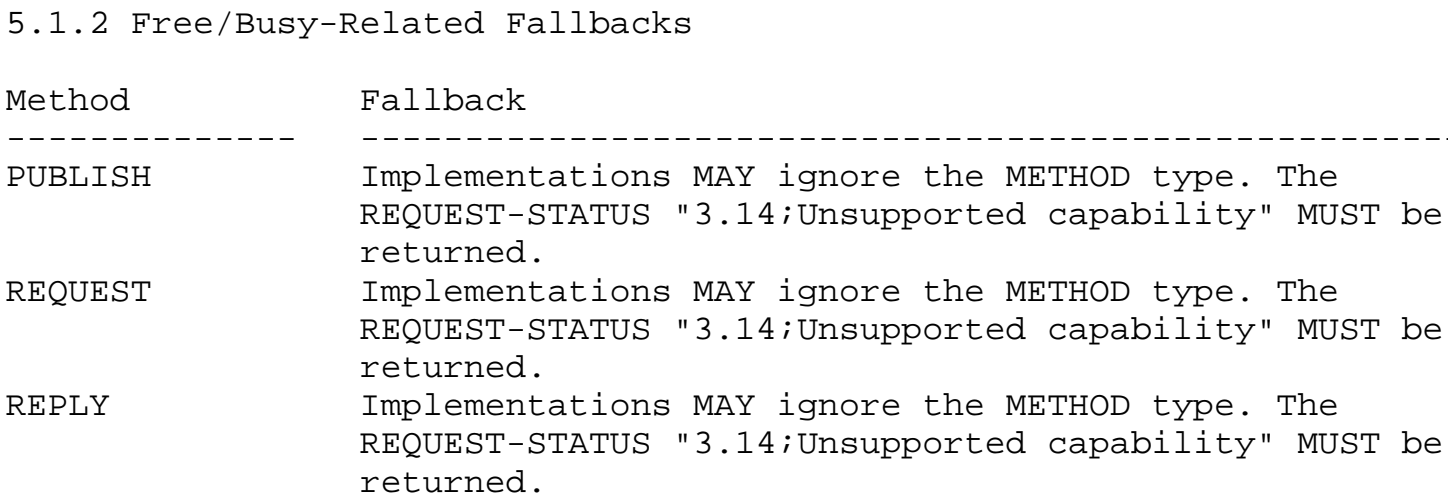

5.1.3 To-Do-Related Fallbacks

\begin{tabular}{ll} 
Method & Fallback \\
\hline---------- & ------------------------------------------- \\
PUBLISH & Required \\
REQUEST & PUBLISH \\
REPLY & Required \\
ADD & Required
\end{tabular}




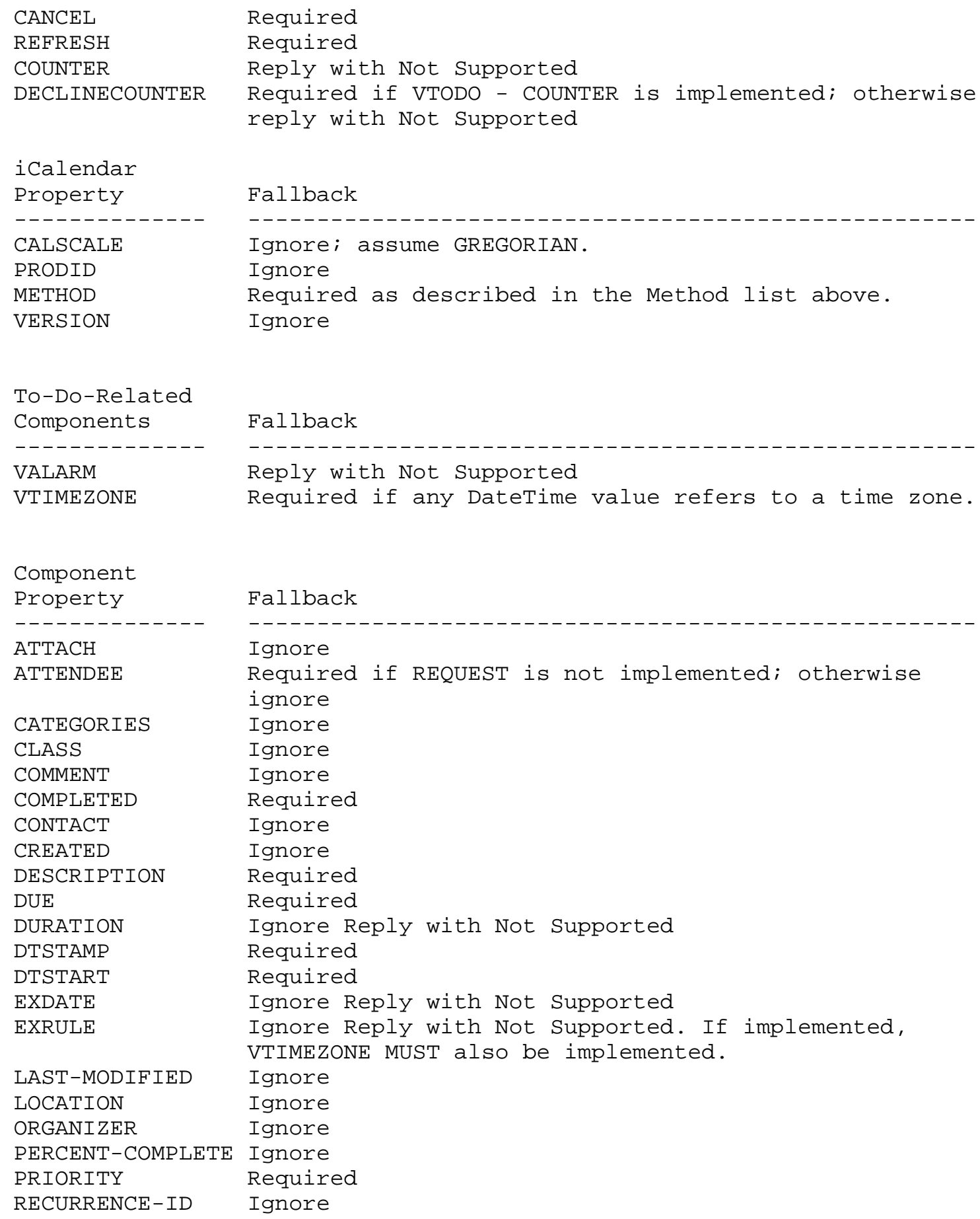

Silverberg, et. al.

Standards Track 


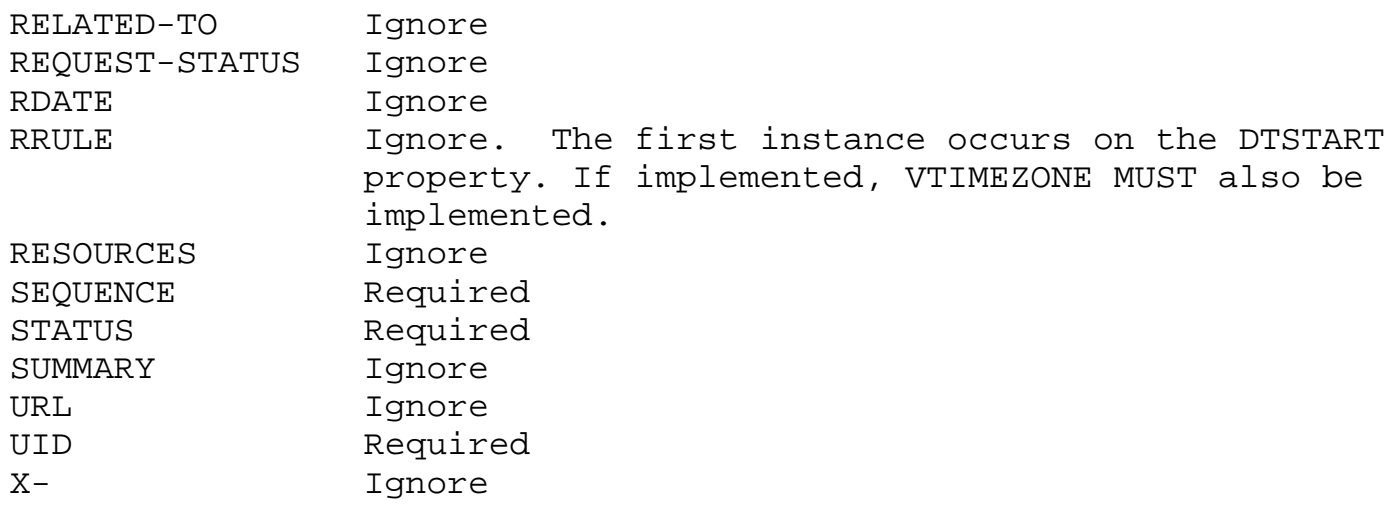


Component

Property

$-------------$

ATTACH

ATTENDEE

CATEGORIES

CLASS

COMMENT

CONTACT

CREATED

DESCRIPTION

DTSTAMP

DTSTART

EXDATE

EXRULE

LAST-MODIFIED

ORGANIZER

RECURRENCE-ID

RELATED-TO

RDATE

RRULE

SEQUENCE

STATUS

SUMMARY

URL

UID

$\mathrm{X}-$
Fallback

Ignore

Required if JOURNAL-REQUEST is implemented; otherwise ignore

Ignore

Ignore

Ignore

Ignore

Ignore

Required

Required

Required

Ignore

Ignore Reply with Not Supported. If implemented, VTIMEZONE MUST also be implemented.

Ignore

Ignore

Ignore

Ignore

Ignore.

Ignore. The first instance occurs on the DTSTART property. If implemented, VTIMEZONE MUST also be implemented.

Required

Ignore

Required

Ignore

Required

Ignore

\subsection{Latency Issues}

With a store-and-forward transport, it is possible for events to arrive out of sequence. That is, a "CANCEL" method may be received prior to receiving the associated "REQUEST" for the calendar component. This section discusses a few of these scenarios.

\subsubsection{Cancellation of an Unknown Calendar Component.}

When a "CANCEL" method is received before the original "REQUEST" method the calendar will be unable to correlate the "UID" property of the cancellation with an existing calendar component. It is suggested that messages that can not be correlated that also contain non-zero sequence numbers be held and not discarded. Implementations MAY age them out if no other messages arrive with the same "UID" property value and a lower sequence number. 


\subsubsection{Unexpected Reply from an Unknown Delegate}

When an "Attendee" delegates an item to another CU they MUST send a "REPLY" method to the "Organizer" using the "ATTENDEE" properties to indicate that the request was delegated and to whom. Hence, it is possible for an "Organizer" to receive an "REPLY" from a CU not listed as one of the original "Attendees". The resolution is left to the implementation but it is expected that the calendaring software will either accept the reply or hold it until the related "REPLY" method is received from the "Delegator". If the version of the "REPLY" method is out of date the "Organizer" SHOULD treat the message as a "REFRESH" message and update the delegate with the correct version.

\subsection{Sequence Number}

Under some conditions, a CUA may receive requests and replies with the same "SEQUENCE" property value. The "DTSTAMP" property is utilized as a tie-breaker when two items with the same "SEQUENCE" property value are evaluated.

6 Security Considerations

ITIP is an abstract transport protocol which will be bound to a real-time transport, a store-and-forward transport, and perhaps other transports. The transport protocol will be responsible for providing facilities for authentication and encryption using standard Internet mechanisms that are mutually understood between the sender and receiver.

\subsection{Security Threats}

\subsubsection{Spoofing the "Organizer"}

In iTIP, the "Organizer" (or someone working on the "Organizer's" behalf) is the only person authorized to make changes to an existing "VEVENT", "VTODO", "VJOURNAL" calendar component and republish it or redistribute updates to the "Attendees". An iCalendar object that maliciously changes or cancels an existing "VEVENT", "VTODO" or "VJOURNAL" calendar component may be constructed by someone other than the "Organizer" and republished or sent to the "Attendees".

\subsubsection{Spoofing the "Attendee"}

In iTIP, an "Attendee" of a "VEVENT" or "VTODO" calendar component (or someone working on the "Attendee's" behalf) is the only person authorized to update any parameter associated with their "ATTENDEE" property and send it to the "Organizer". An iCalendar object that 
maliciously changes the "ATTENDEE" parameters may be constructed by someone other than the real "Attendee" and sent to the "Organizer".

\subsubsection{Unauthorized Replacement of the Organizer}

There will be circumstances when "Attendees" of an event or to-do decide, using out-of-band mechanisms, that the "Organizer" must be replaced. When the new "Organizer" sends out the updated "VEVENT" or "VTODO" the "Attendee's" CUA will detect that the "Organizer" has been changed, but it has no way of knowing whether or not the change was mutually agreed upon.

\subsubsection{Eavesdropping}

The icalendar object is constructed with human-readable clear text. Any information contained in an icalendar object may be read and/or changed by unauthorized persons while the object is in transit.

\section{1 .5 Flooding a Calendar}

Implementations MAY provide a means to automatically incorporate "REQUEST" methods into a calendar. This presents the opportunity for a calendar to be flooded with requests, which effectively block all the calendar's free time.

\subsubsection{Procedural Alarms}

The "REQUEST" methods for "VEVENT" and "VTODO" calendar components MAY contain "VALARM" calendar components. The "VALARM" calendar component may be of type "PROCEDURE" and MAY have an attachment containing an executable program. Implementations that incorporate these types of alarms are subject to any virus or malicious attack that may occur as a result of executing the attachment.

\subsubsection{Unauthorized REFRESH Requests}

It is possible for an "Organizer" to receive a "REFRESH" request from someone who is not an "Attendee" of an event or to-do. Only "Attendee's" of an event or to-do are authorized to receive replies to "REFRESH" requests. Replying to such requests to anyone who is not an "Attendee" may be a security problem.

\subsection{Recommendations}

For an application where the information is sensitive or critical and the network is subject is subject to a high probability of attack, iTIP transactions SHOULD be encrypted. This may be accomplished using public key technology, specifically Security Multiparts for MIME 
[RFC-1847] in the iTIP transport binding. This helps mitigate the threats of spoofing, eavesdropping and malicious changes in transit.

6.2.1 Use of [RFC-1847] to secure iTIP transactions

iTIP transport bindings MUST provide a mechanism based on Security Multiparts for MIME [RFC-1847] to enable authentication of the sender's identity, and privacy and integrity of the data being transmitted. This allows the receiver of a signed icalendar object to verify the identity of the sender. This sender may then be correlated to an "ATTENDEE" property in the iCalendar object. If the correlation is made and the sender is authorized to make the requested change or update then the operation may proceed. It also allows the message to be encrypted to prevent unauthorized reading of the message contents in transit. iTIP transport binding documents describe this process in detail.

Implementations MAY provide controls for users to disable this capability.

\subsubsection{Implementation Controls}

The threat of unauthorized replacement of the "Organizer" SHOULD be mitigated by a calendar system that uses this protocol by providing controls or alerts that make "Calendar Users" aware of such "Organizer" changes and allowing them to decide whether or not the request should be honored.

The threat of flooding a calendar SHOULD be mitigated by a calendar system that uses this protocol by providing controls that may be used to limit the acceptable sources for iTIP transactions, and perhaps the size of messages and volume of traffic, by source.

The threat of malicious procedural alarms SHOULD be mitigated by a calendar system that uses this protocol by providing controls that may be used to disallow procedural alarms in iTIP transactions and/or remove all alarms from the object before delivery to the recipient.

The threat of unauthorized "REFRESH" requests SHOULD be mitigated by a calendar system that uses this protocol by providing controls or alerts that allow "Calendar User" to decide whether or not the request should be honored. An implementation MAY decide to maintain, for audit or historical purposes, "Calendar Users" who were part of an attendee list and who were subsequently uninvited. Similar controls or alerts should be provided when a "REFRESH" request is received from these "Calendar Users" as well. 
7 Acknowledgments

A hearty thanks to the following individuals who have participated in the drafting, review and discussion of this memo:

Anik Ganguly, Dan Hickman, Paul Hill, Daryl Huff, Bruce Kahn, Antoine Leca, Bob Mahoney, John Noerenberg, Leo Parker, John Rose, Doug Royer, Vinod Seraphin, Richard Shusterman, Derik Stenerson, John Sun, Alexander Taler, Kevin Tsurutome.

8 Bibliography

[iCAL] Dawson, F. and D. Stenerson, "Internet Calendaring and Scheduling Core Object Specification - iCalendar", RFC 2445, November 1998 .

[iMIP] Dawson, F., Mansour, S. and S. Silverberg, "iCalendar Message-Based Interoperability Protocol - iMIP", RFC 2447, November 1998 .

[RFC-2119] Bradner, S., "Key words for use in RFCs to Indicate Requirement Levels", BCP 14, RFC 2119, March 1997.

[US-ASCII] Coded Character Set--7-bit American Standard Code for Information Interchange, ANSI X3.4-1986. 
9 Authors' Addresses

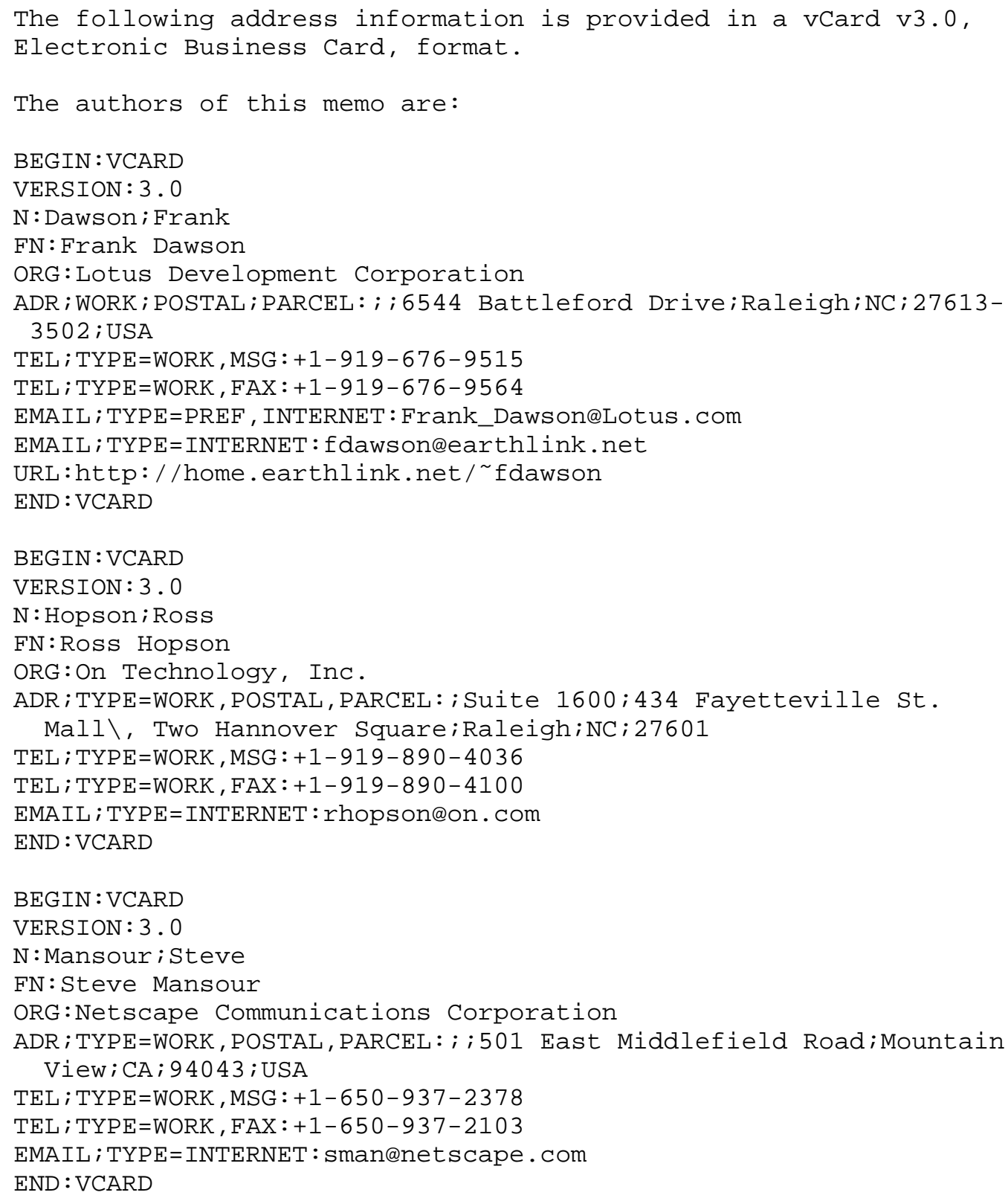




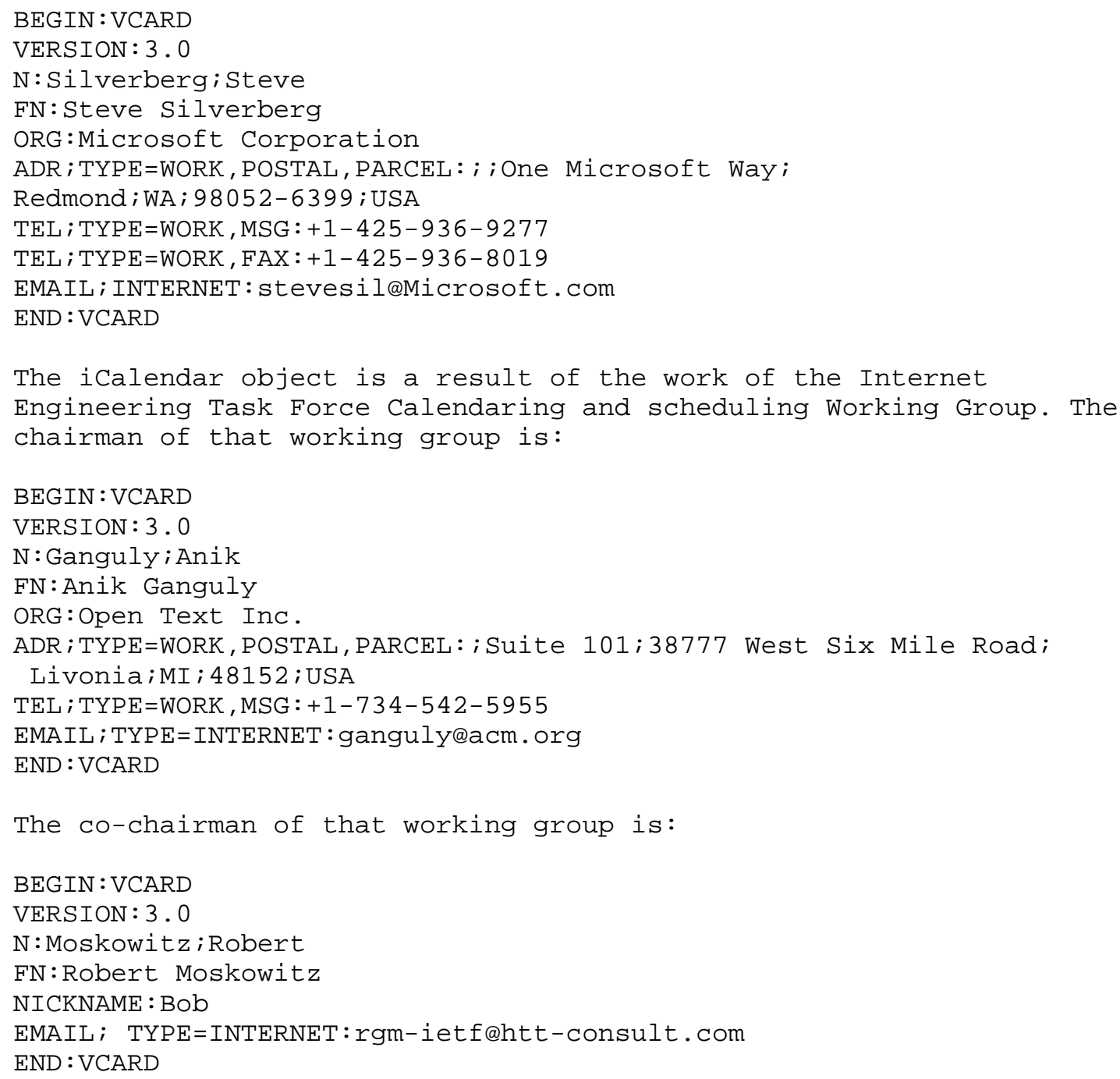


10. Full Copyright statement

Copyright (C) The Internet Society (1998). All Rights Reserved.

This document and translations of it may be copied and furnished to others, and derivative works that comment on or otherwise explain it or assist in its implementation may be prepared, copied, published and distributed, in whole or in part, without restriction of any kind, provided that the above copyright notice and this paragraph are included on all such copies and derivative works. However, this document itself may not be modified in any way, such as by removing the copyright notice or references to the Internet society or other Internet organizations, except as needed for the purpose of developing Internet standards in which case the procedures for copyrights defined in the Internet Standards process must be followed, or as required to translate it into languages other than English.

The limited permissions granted above are perpetual and will not be revoked by the Internet society or its successors or assigns.

This document and the information contained herein is provided on an "AS IS" basis and THE INTERNET SOCIETY AND THE INTERNET ENGINEERING TASK FORCE DISCLAIMS ALL WARRANTIES, EXPRESS OR IMPLIED, INCLUDING BUT NOT LIMITED TO ANY WARRANTY THAT THE USE OF THE INFORMATION HEREIN WILL NOT INFRINGE ANY RIGHTS OR ANY IMPLIED WARRANTIES OF MERCHANTABILITY OR FITNESS FOR A PARTICULAR PURPOSE. 